\title{
Big Brother: A Drop-In Website Interaction Logging Service
}

\author{
Harrisen Scells \\ h.scells@uq.net.au \\ The University of Queensland \\ Brisbane, Australia
}

\author{
Jimmy \\ jimmy@ubaya.ac.id \\ University of Surabaya \\ Surabaya, Indonesia
}

\author{
Guido Zuccon \\ g.zuccons@uq.edu.au \\ The University of Queensland \\ Brisbane, Australia
}

\begin{abstract}
Fine-grained logging of interactions in user studies is important for studying user behaviour, among other reasons. However, in many research scenarios, the way interactions are logged are usually tied to a monolithic system. We present a generic, applicationindependent service for logging interactions in web-pages, specifically targetting user studies. Our service, Big Brother, can be dropped-in to existing user interfaces with almost no configuration required by researchers. Big Brother has already been used in several user studies to record interactions in a number of user study research scenarios, such as lab-based and crowdsourcing environments. We further demonstrate the ability for Big Brother to scale to very large user studies through benchmarking experiments. Big Brother also provides a number of additional tools for visualising and analysing interactions.

Big Brother significantly lowers the barrier to entry for logging user interactions by providing a minimal but powerful, no configuration necessary, service for researchers and practitioners of user studies that can scale to thousands of concurrent sessions. We have made the source code and releases for Big Brother available for download at https://github.com/hscells/bigbro.
\end{abstract}

\section{CCS CONCEPTS}

- Information systems $\rightarrow$ Traffic analysis.

\section{KEYWORDS}

user studies, interaction logging

\section{ACM Reference Format:}

Harrisen Scells, Jimmy, and Guido Zuccon. 2021. Big Brother: A Drop-In Website Interaction Logging Service. In Proceedings of the 44th International ACM SIGIR Conference on Research and Development in Information Retrieval (SIGIR '21), July 11-15,2021, Virtual Event, Canada. ACM, New York, NY, USA, 5 pages. https://doi.org/10.1145/3404835.3462781

\section{PROBLEM, TARGET USERS \& IMPORTANCE}

Recording interactions in a user study setting is extremely important for understanding user behaviour, among other reasons. However, writing the code to implement the recording of interactions can be tedious and a burden on those who wish to run user studies.

Permission to make digital or hard copies of all or part of this work for personal or classroom use is granted without fee provided that copies are not made or distributed for profit or commercial advantage and that copies bear this notice and the full citation on the first page. Copyrights for components of this work owned by others than the author(s) must be honored. Abstracting with credit is permitted. To copy otherwise, or republish, to post on servers or to redistribute to lists, requires prior specific permission and/or a fee. Request permissions from permissions@acm.org.

SIGIR'21, fuly 11-15, 2021, Virtual Event, Canada

(c) 2021 Copyright held by the owner/author(s). Publication rights licensed to ACM. ACM ISBN 978-1-4503-8037-9/21/07 ..\$15.00

https://doi.org/10.1145/3404835.3462781

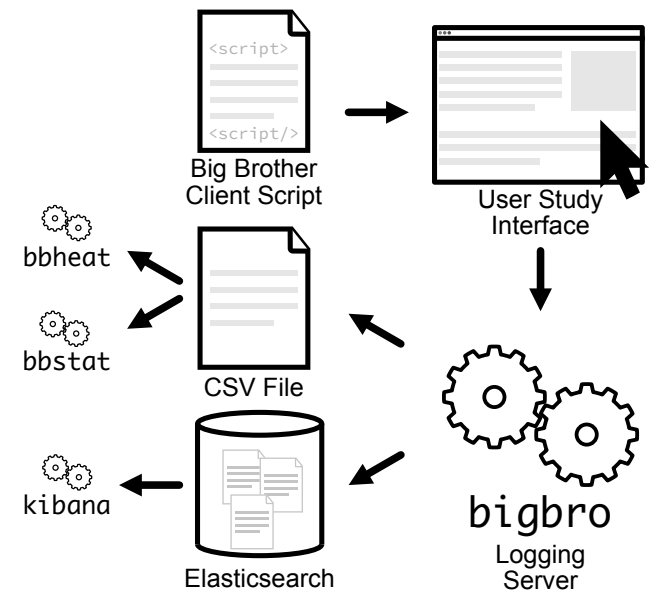

Figure 1: High-level overview of the system architecture. Indeed, there is a considerable amount of software infrastructure to be considered when logging user interactions: How to efficiently process streams of interactions from multiple users/sessions? How to store the logs? How to deal with all the types of interactions a user might make? When we encountered these questions, it was clear that a standard, simple, and drop-in service was required. The requirement for the service to passively log interactions once loaded into a web page also provides a generic way to capture events from users no matter the framework or setting that the user study is run. We present this service to the wider IR community as an Open Source tool, dubbed Big Brother. An overview of the Big Brother architecture is presented in Figure 1.

Fine-grained interaction logs enable researchers to more deeply understand how users behave in their systems. Tracking and analysing how users behave is important for a number of reasons:

Evaluation. While users self-reported measurements can provide a weak indication of the effort required to complete tasks, tracking user interactions can provide a stronger source of evidence for how much effort, or how challenging a user found a task. For example, by logging user interactions with Big Brother, Cross et al. [2] found that user reported effort differed from the number of interactions between two systems.

User Modelling. Recording how users interact with systems enables a deeper understanding of user behaviour, and can lead to models that better reflect the needs of users. In Information Retrieval tasks, White [7] notes that modelling user behaviour permits "better understanding the search process, estimating satisfaction, identifying connections between queries and/or URLs, and learning which results are relevant."

Multi-Modal Interactions. Often it is desirable to analyse behavioural patterns between how users interact with the keyboard 
and mouse and other physiological interactions, such as e facial expressions [1]. For example, using Big Brother, Jimmy et al. [4] were able to better understand what users paid attention to by combining interaction data like clicks and mouse movements with eye-tracking.

Malicious Users. By tracking how frequently a user interacts with elements on a page it is possible to identify behavioural patterns that may correspond with malicious intent [3].

\section{OPERATION OF THE SYSTEM}

Big Brother has two main components: a high-throughput server and a JavaScript client. The client listens to events that occur in a web browser, and the server ingests them in real-time.

\subsection{Client}

The Big Brother client has been written to provide maximum functionality with minimal setup and configuration. The smallest amount of code to have Big Brother listen to events is presented in Listing 1. By default, Big Brother is configured to listen to all events on all elements on a page. This client has been put to the test on multiple user studies with highly complex interfaces [2, 4, 5]. Practitioners of these studies and users of their systems noticed no slowdown of their study as a result of using Big Brother.

Listing 1: Minimal example of code required to initialise bigbro on the client-side. Note the session_id which should already be initialised elsewhere.

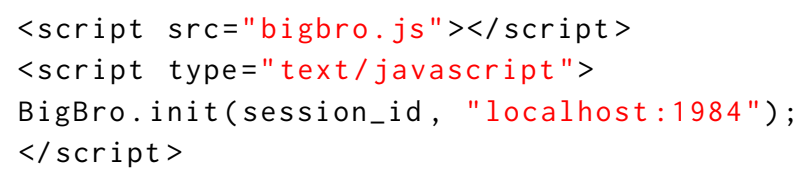

Often, however, the practitioner of a user study may only be interested in a subset of events. Listing 2 presents the JavaScript code required on the client-side to restrict Big Brother to listen to two events: mousemove and onload, which will record the coordinates of the users' mouse, and the time the session began respectively.

Listing 2: Initialising bigbro to listen on only certain global events. HTML code removed for brevity.

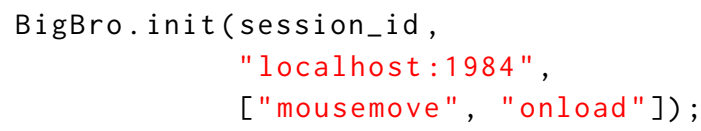

Sometimes, the practitioner of a user study may wish to capture custom interactions or events from their user. For example, logging a custom event for when a user clicks a specific button on the page. Listing 3 presents the JavaScript code required on the client-side to wire the processing of a custom event to Big Brother's exposed custom logging functionality.

Listing 3: Wiring up bigbro to listen to click events and to $\log$ a custom event.

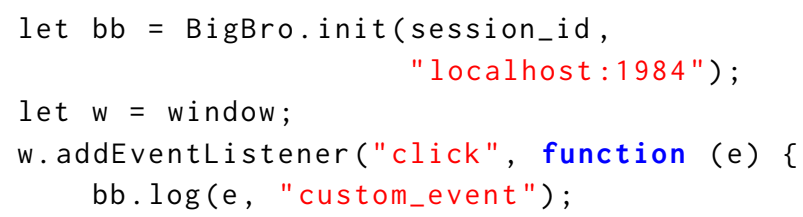

\begin{tabular}{|c|c|}
\hline Component & Description of Component \\
\hline Target & $\begin{array}{l}\text { The HTML element that has caused the event } \\
\text { to trigger. }\end{array}$ \\
\hline Name & $\begin{array}{l}\text { The name attribute of the HTML element in } \\
\text { Target. }\end{array}$ \\
\hline ID & The id attribute of the HTML element in Target \\
\hline Method & $\begin{array}{l}\text { The name of the method that caused the event } \\
\text { to trigger. }\end{array}$ \\
\hline Location & $\begin{array}{l}\text { The web-page location on the server (URL, with } \\
\text { query string and anchors). }\end{array}$ \\
\hline Comment & $\begin{array}{l}\text { Any additional custom information that may } \\
\text { be useful to interpreting the event. }\end{array}$ \\
\hline $\mathrm{X}$ & $\begin{array}{l}\text { The x-position within the web browser that the } \\
\text { event occurred at. }\end{array}$ \\
\hline $\mathrm{Y}$ & $\begin{array}{l}\text { The y-position within the web browser that the } \\
\text { event occurred at. }\end{array}$ \\
\hline ScreenWidth & $\begin{array}{l}\text { The width of the web browser the event oc- } \\
\text { curred within. }\end{array}$ \\
\hline ScreenHeight & $\begin{array}{l}\text { The height of the web browser the event oc- } \\
\text { curred within. }\end{array}$ \\
\hline Time & The time the event happened. \\
\hline Actor & $\begin{array}{l}\text { An identifier that can be used to refer to the } \\
\text { user that caused the event, e.g., session ID. }\end{array}$ \\
\hline
\end{tabular}

Table 1: The components of an event.

\})

Finally, it is possible to record the screen using Big Brother. Listing 4 presents the minimum JavaScript code required to start a live capture of the browser window a user is looking at. Because of browser security, screen capture cannot be started automatically, and must be initiated by clicking an element (as seen in the argument provided to the function in Listing 4). Captures are saved as a series of images. The time between each capture can be configured, and captures can be taken in response to events. The images can be stitched together into a video later, or can be used to highlight specific interactions from the logs.

Listing 4: Wiring up bigbro to listen to click events and to $\log$ a custom event.

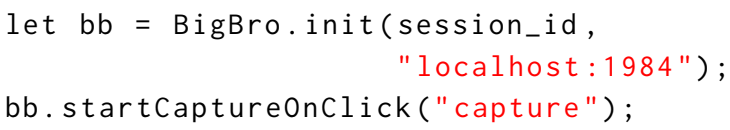

The Big Brother client has been specifically designed to require very minimal configuration and setup. Likewise, the server is designed to be run independently of components such as the web server a user study is hosted on.

\subsection{Server}

The server is written in the Go programming language. This provides a number of advantages, primarily the ability for the server code to be compiled into a high-performance single-binary file for multiple platforms (macOS, Windows, Linux, etc.). The server can be configured using command-line options. It can also be integrated into existing web servers as library code, so long as those servers are written in Go. 
The server processes interactions from users as events. The components that comprise an event are summarised in Table 1. An event captures information about the HTML elements that were interacted with (Target, Name, ID), how the event was triggered (Method), what the URL was on the page that the event occurred (Location), additional custom comments (Comment), positional information to "replay" interactions (X, Y, ScreenWidth, ScreenHeight), the time that the event occurred (Time), and the user that caused the event (Actor).

Events are streamed from the user's web browser to a centralised server over websockets. This enables the real-time ingestion of interactions. Currently, Big Brother can output logs to a local csv file as well as directly indexing them to Elasticsearch. A small, real example of logs captured during a user study is presented in Listing 5. The researcher who ran this study decided to output their interaction logs directly to a csv file. The ordering of components in these events is how they are produced by bigbro. Listing 6 shows the ordering of components in the csv file output. These logs show the interactions of two user sessions from a human intelligence task (HIT) on a popular crowdsourcing platform. This slice of interaction logs begins when the A1 user starts a HIT, and ends when they finish the HIT. At the same time, another user (A2) is in the middle of another HIT. The researcher running this user study records when their users click on a multiple select dropdown. The very final line in the log, when user A1 finishes the HIT, also logs the recorded answers to the questionnaire using the Comment component: $\mathrm{T}|\mathrm{T}| \mathrm{T}|\mathrm{T}| \mathrm{T}$.

In addition to allowing the researcher to see in real-time how users are interacting with their experiments (and potentially throw away sessions from users who completed the study too quickly), it provides a second way to record responses that users make to questionnaires.

\subsection{Throughput Benchmark}

We demonstrate the ability of Big Brother to scale and accommodate very large user studies by providing a throughput benchmark of logging user interactions directly to a csv file. To make the benchmark more realistic, we simulate the time it takes to process an event by encoding and decoding the event, and populate the event with random data. Each event has Target, Method, Location, X, Y, ScreenHeight, ScreenWidth, Time and Actor components. To benchmark the code, we use the standard benchmarking framework included in the Go language (as this is the language that the server is written in). In this framework, a loop containing the process described earlier is run $N$ times until it takes a second for the benchmark to complete. We report the total number of events processed, the average time it takes to process one event in microseconds, and the throughput in $\mathrm{MB} / \mathrm{s}$. We run the benchmark ten times and report both each run and the overall statistics. The benchmarks were run on a $2.7 \mathrm{GHz}$ Intel Core i7 8-core 2018 MacBook Pro with 16GB of LPDDR3 RAM and solid-state flash storage. Table 2 presents the results of the benchmark. On average, Big Brother can process approximately 70,000 events per second, with each event processed in approximately $26 \mu \mathrm{s}$, and write the interactions to a file at a speed of approximately $5 \mathrm{MB} / \mathrm{s}$. In practice, while we never came close to this amount of interactions per second, bigbro did remain resilient and experienced no faults over the course of a number of studies.

\begin{tabular}{lll}
\hline Processed Events & $\mu$ s/Event & Throughput $(\mathrm{MB} / \mathrm{s})$ \\
\hline 79,026 & 22.21 & 5.58 \\
71,455 & 29.51 & 4.27 \\
37,600 & 32.79 & 3.87 \\
84,356 & 31.22 & 4.07 \\
60,418 & 19.31 & 6.53 \\
82,922 & 23.70 & 5.36 \\
72,714 & 30.97 & 4.10 \\
83,862 & 27.30 & 4.61 \\
86,094 & 24.86 & 5.07 \\
51,250 & 24.37 & 5.21 \\
\hline \hline $70,969.70 \pm 16,326.29$ & $26.60 \pm 0.27$ & $4.87 \pm 0.34$
\end{tabular}

Table 2: Benchmark results of logging events. The last row indicates average across the runs, with standard deviation. The processed events column indicates the number of events processed in one second.

\subsection{Other Tools within Big Brother}

The Big Brother family also includes other tools for visualising and manipulating data produced by the bigbro tool. These include bbheat and bbstat, which are compatible with CSV formatted logs produced by bigbro. If Elasticsearch is used as the storage medium for logs, it is possible to use the infrastructure that has been developed for it, including Kibana, a data visualization dashboard. Note that it is possible to use the Big Brother tools and the Elasticsearch tools by allowing bigbro to write to a CSV file and ingesting that file into Elasticsearch using software such as Logstash. For details on how to use these tools, please refer to the GitHub repository.

\section{5 bbheat}

Often it is useful to be able to visualise the interactions of users over user interfaces. For this, the bbheat tool can be used. This tool allows one to produce a static or animated heat map of mouse movements. The tool has several options for filtering interactions, for example by actor, time, and browser window size. An example of one of the heat maps produced by this tool is presented in Figure 2. Using the screen recording functionality of Big Brother, it becomes trivial to visualise how different users interact with a system, or how changes in interface design can impact user behaviour.

\section{6 bbstat}

Analysing log data can be tedious, especially processing the data for analysis. The bbstat tool provides common functionality for processing interaction logs for later analysis. The functionality provided by the bbstat tool include filtering: interaction logs can be split into multiple files based on actor, time, or comment, and aggregation: production of reports such as how long a user spent on different pages or how many times certain elements in a page were clicked.

\subsection{Kibana}

Kibana is a product that is developed by the same company that built Elasticsearch. It provides tools for visualising and manipulating data. Kibana has been developed with time series logs in mind. A deeper description of Kibana is not provided here for space reasons, but it can be a powerful alternative to the tools provided by Big Brother. 
Listing 5: Example log file capturing two user sessions at the same time. Note that there were far more interactions captured during the study and that this is a small contrived slice of the data. Much of the information from the URL has been redacted.

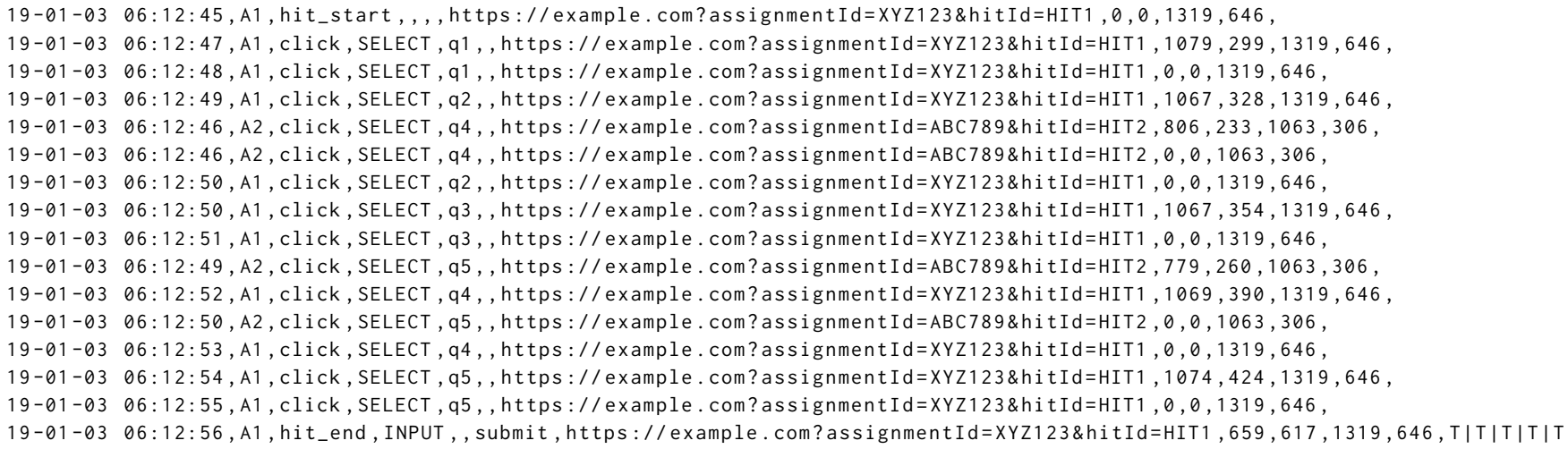

Listing 6: Order of event components in Listing 5

Time, Actor, Method, Target, Name, ID, Location, X, Y, ScreenWidth, ScreenHeight, Comment

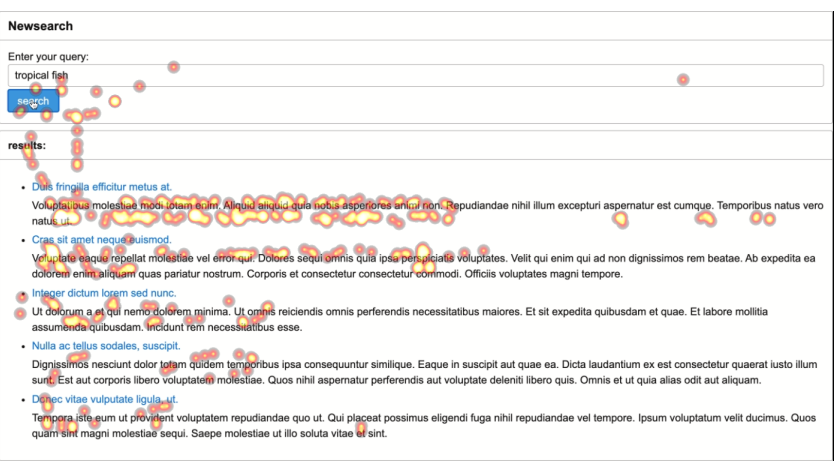

Figure 2: Heat map of mouse interactions overlaid on top of an image of a search interface captured using the screen recording functionality of Big Brother.

\section{COMPARISON WITH EXISTING SYSTEMS}

The idea to capture interactions in user study scenarios is not new. For example, Zuccon et al. [8] proposed a system for evaluating Information Retrieval systems using interactions from crowdsourced workers. They observe interactions such as the length of sessions, the number of elements clicked, and the number of items examined. However, they do not measure fine-grained interactions such as mouse movements, and these measurements are built into the underlying interface, unlike Big Brother which can log interactions independently of the underlying interface. Bierig et al. [? ] proposed an end-to-end system for designing user studies and logging multi-modal interactions within those studies. The downside to this system is that it may not provide the flexibility afforded by bespoke user study systems. Big Brother is able to be integrated easily into highly bespoke web-based systems. Atterer and Albrecht [? ] proposed a similar interaction logging system to ours by using AJAX requests instead of web sockets. However, their events contain fewer components than ours, and it is unlikely that this older technology would scale for large user studies of today. More recently, Maxwell and Hauff [6] have developed fine-grained logging infrastructure for web-based experiments that use similar modern technology to ours. While the architecture of their system may be similar to ours, we believe that the barrier to entry for logging interactions is significantly lower using Big Brother and the fact that Big Brother has been used already in a number of user studies (lab-based and embedded inside crowdsourcing services) demonstrates its robustness in different user study scenarios. We further contrast Big Brother to their LogUI system by demonstrating the additional ability to record the screen of users and the associated tools for visualising and manipulating interactions.

Lettner and Holzmann [?] and Kokemore and Hutter [?] both proposed toolkits for automatically logging user interactions in mobile applications. Their frameworks differ from ours as they target mobile settings (e.g., Android or iOS apps) instead of webbased interfaces. Note however that Big Brother is compatible with touch events in a mobile browser. In a similar vein, Jeong et al. [?] proposed a framework for visualising how users transition from one mobile application screen to the next. This system captures a screenshot each time the user transitions to a new interface in the app. This functionality is exposed in Big Brother, but this system can provide a transition graph visualisation, which we do not.

\section{IMPACT OF BIG BROTHER}

The Big Brother logging system has been used already in several user studies and has been 'battle tested'. We demonstrate the ability of the bigbro server to scale to tens of thousands of interactions per second. We have developed the Big Brother ecosystem to allow researchers to integrate interaction logging into any web-based system. Furthermore, we have designed the entire system to be extremely easy to use, while providing highly sophisticated functionality, such as screen recording. These attributes, we believe, will significantly lower the barrier to entry for capturing user interactions in many research settings.

Acknowledgements. We would like to thank Anton van der Vegt and Sebastian Cross for their use of Big Brother in their user studies and Ahmed Mourad for proofreading a draft of this paper. Dr Guido Zuccon is the recipient of an Australian Research Council DECRA Research Fellowship (DE180101579) and Google Faculty Award. This research is supported by the Grain Research and Development Corporation (GRDC), project AgAsk (UOQ2003-009RTX). 


\section{REFERENCES}

[1] Ioannis Arapakis, Joemon M Jose, and Philip D Gray. 2008. Affective Feedback: An Investigation into the Role of Emotions in the Information Seeking Process. In Proceedings of the 31st Annual International ACM SIGIR Conference on Research and Development in Information Retrieval. 8.

[2] Sebastian Cross, Ahmed Mourad, Guido Zuccon, and Bevan Koopman. 2021. Search Engines vs. Symptom Checkers: A Comparison of Their Effectiveness for Online Health Advice. In Proceedings of the 2021 Web Conference. 11

[3] Ujwal Gadiraju, Ricardo Kawase, Stefan Dietze, and Gianluca Demartini. 2015. Understanding Malicious Behavior in Crowdsourcing Platforms: The Case of Online Surveys. In Proceedings of the 33rd Annual ACM Conference on Human Factors in Computing Systems. ACM, Seoul Republic of Korea, 1631-1640.

[4] Jimmy, Guido Zuccon, Gianluca Demartini, and Bevan Koopman. 2020. Health Cards to Assist Decision Making in Consumer Health Search. In AMIA Annual
Symposium Proceedings, Vol. 2019. 1091-1100.

[5] Jimmy Jimmy, Guido Zuccon, Bevan Koopman, and Gianluca Demartini. 2019. Health Cards for Consumer Health Search. In Proceedings of the 42nd International ACM SIGIR Conference on Research and Development in Information Retrieval. ACM, $35-44$.

[6] David Maxwell and Claudia Hauff. 2021. LogUI: Contemporary Logging Infrastructure for Web-Based Experiments. In Proceedings of the 43rd European Conference on Information Retrieval.

[7] Ryen W. White. 2016. Interactions with Search Systems. Cambridge University Press

[8] Guido Zuccon, Teerapong Leelanupab, Stewart Whiting, Emine Yilmaz, Joemon M. Jose, and Leif Azzopardi. 2013. Crowdsourcing Interactions: Using Crowdsourcing for Evaluating Interactive Information Retrieval Systems. Information retrieval 16, 2 (2013), 267-305. 
Association for

Computing Machinery

July 11-15, 2021

Virtual Event, Canada

Advancing Computing as a Science \& Profession

\section{SIGIR' 21}

Proceedings of the 44th International ACM SIGIR Conference on Research and Development in Information Retrieval

Sponsored by:

ACM SIGIR

General Chairs:

Fernando Diaz, Chirag Shah, \& Torsten Suel

Program Chairs:

Pablo Castells, Rosie Jones, \& Tetsuya Sakai

Proceedings Chairs:

Alejandro Bellogín \& Masaharu Yoshioka 


\title{
Association for Computing Machinery
}

\author{
Advancing Computing as a Science \& Profession \\ The Association for Computing Machinery \\ 1601 Broadway, $10^{\text {th }}$ Floor \\ New York, NY 10019-7434
}

Copyright (C) 2021 by the Association for Computing Machinery, Inc. (ACM). Permission to make digital or hard copies of portions of this work for personal or classroom use is granted without fee provided that copies are not made or distributed for profit or commercial advantage and that copies bear this notice and the full citation on the first page. Copyright for components of this work owned by others than ACM must be honored. Abstracting with credit is permitted. To copy otherwise, to republish, to post on servers or to redistribute to lists, requires prior specific permission and/or a fee. Request permission to republish from: permissions@acm.org or Fax +1 (212) 869-0481.

For other copying of articles that carry a code at the bottom of the first or last page, copying is permitted provided that the per-copy fee indicated in the code is paid through www.copyright.com.

ISBN: 978-1-4503-8037-9

Additional copies may be ordered prepaid from:

ACM Order Department

PO Box 30777

New York, NY 10087-0777, USA

Phone: 1-800-342-6626 (USA and Canada)

+1-212-626-0500 (Global)

Fax: +1-212-944-1318

E-mail: acmhelp@acm.org

Hours of Operation: 8:30 am - 4:30 pm ET

Printed in the USA 


\section{ACM SIGIR 2021 Chairs' Welcome}

Welcome to the 44th Annual International ACM SIGIR Conference on Research and Development in Information Retrieval (SIGIR 2021). SIGIR is the premier scientific conference in the broad area of information retrieval. SIGIR 2021 was originally planned to be held in Montréal, Quebec, but, due to the global pandemic, had to be shifted to a virtual conference.

In addition to the traditional technical program familiar to the SIGIR community, SIGIR 2021 has successfully launched two new tracks: Resource and Perspectives. Across all tracks, SIGIR continues to grow in terms of the number of submissions, as shown in the table below.

\begin{tabular}{|l|c|c|c|}
\hline & Valid submissions & Accepted & Acceptance rate (\%) \\
\hline Full papers & 720 & 151 & 20.9 \\
\hline Short papers & 526 & 145 & 27.6 \\
\hline Demo papers & 39 & 21 & 53.8 \\
\hline Resource papers (new track) & 60 & 27 & 45.0 \\
\hline Perspectives papers (new track) & 11 & 3 & 27.3 \\
\hline SIRIP papers & 20 & 9 & 45.0 \\
\hline Doctoral consortium papers & 13 & 11 & 84.6 \\
\hline Workshop proposals & 9 & 6 & 66.7 \\
\hline Tutorial proposals & 12 & 9 & 75.0 \\
\hline
\end{tabular}

Valid submissions came from a total of 6,335 authors from 54 countries. We are thankful to all of these authors for choosing to submit to SIGIR 2021. In response to these high-quality papers, 976 reviewers provided reviews and meta-reviews, resulting in a total of 4,241 reviews and meta reviews. We thank the program committees for their diligence. The accepted papers and proposals are contributions from a total of 2,454 authors from 40 countries.

SIGIR 2021's technical program is highlighted by three invited keynote presentations and the 2021 Salton Award keynote. We thank our invited speakers, Eszter Hargittai (University of Zurich, Switzerland), Hang Li (ByteDance, China), and Helen Nissenbaum (Cornell University, USA), for sharing their scholarship and perspective with the SIGIR community.

Adding to this year's agenda is the colocation of the ICTIR 2021 conference, 9 tutorials, 6 workshops, two days of SIRIP, and newly established SIGIR Academy inductions. This year's conference program also includes presentations for 37 papers from ACM Transactions on Information Systems, Volumes 37 and 38, continuing the tradition of supporting knowledge transfer. We are also continuing with events such as Women in IR and a panel on Diversity, Equity, and Inclusion (DEI).

But we are doing more than just a panel discussion on DEI this year. Taking advantage of the online format and to continue making SIGIR a welcoming and inclusive space, we have paid special attention to DEI efforts. This is reflected in several new programs and initiatives. This starts with having considerably low registration fees across the board. Then, providing significant discounts to those 
coming from least developed countries (as defined by the UN), and giving substantially discounted or free registration to those who need it. This is on top of SIGIR's Student Travel Award (which is used to cover full registration costs for those students). We are also pleased to offer funds to cover costs for familycare, so more people can attend the conference sessions without having to worry about taking care of their dependents or paying for their care.

All of this would not be possible without a stellar team of scholars, leaders, and volunteers putting in exceptional amounts of work and thoughts. We would like to acknowledge the organization team: our Short Paper Chairs, Hui Fang, Claudia Hauff, and Ruihua Song; our Perspectives Chairs, Fernando Diaz and Chirag Shah; our Best Paper Award Chairs, Alistair Moffat and Emine Yilmaz; SIGIR Awards CoChairs who administer the Test of Time Award; our Industry Chairs, Hema Raghavan, Rishabh Mehrotra, and Karthik Subbian; our Social Media Chair, Johanne Trippas; our Doctoral Consortium Chairs, Hiroaki Ohshima and Barbara Poblete; our Workshop Chairs, Abdigani Dirye and Jiafeng Guo; our Demonstration Paper Chairs, Miguel Martínez and Milad Shokouhi; our Tutorial Chairs, Sengor Altingovde and Rodrygo Santos; our Proceedings Chairs, Alejandro Bellogín and Masaharu Yoshioka; our Sponsorship Chairs, Charlie Clarke and Zhaochun Ren; our Virtual Chairs, Bhaskar Mitra and JianYun Nie, who worked to make sure that the online aspects of the conference reflected our community's values; our Registration Chair, Jiqun Liu; our Diversity and Inclusion team, Barbara Poblete, Hussein Suleman, Suzan Verberne, and Bhaskar Mitra; and last but not least our Webmaster, Matthew Mitsui. We would also like to thank ACM for providing logistical and budget support and the SIGIR Executive Committee for their leadership.

In addition, we would like to express our gratitude to our sponsor ACM SIGIR and all our supporters: Baidu, Huawei, Microsoft, Alibaba, Amazon, Apple, Bloomberg, ByteDance, Google, Naver, Sogou, and Spotify.

Finally, we would like to thank the SIGIR Executive Committee for their vote of confidence in us. It is a great pleasure to take on the responsibilities and challenges. We are pleased to offer this excellent program. We hope that attendees find the technical program of SIGIR 2021 to be interesting and productive towards their research endeavors. We further hope that the conference will be stimulating, informative, enjoyable, and a fulfilling and unforgettable experience for all who attend it.

While we are missing an opportunity to meet in person in Montréal as we had planned for, we hope you still get to enjoy the SIGIR 2021 conference in the online, more-inclusive format.

\section{Fernando Diaz \\ SIGIR'21 General Co-Chair \\ Google \\ Canada}

Pablo Castells

SIGIR'21 PC Co- Chair

Universidad Autónoma de Madrid

and Amazon, Spain
Chirag Shah

SIGIR'21 General Co-Chair

University of Washington

USA

Rosie Jones

SIGIR'21 PC Co-Chair

Spotify

USA

\section{Torsten Suel \\ SIGIR'21 General Co-Chair \\ New York University \\ USA}

Tetsuya Sakai

SIGIR'21 PC Co-Chair

Waseda University

Japan 


\section{Table of Contents}

ACM SIGIR 2021 Conference Organization xxxvi

SIGIR 2021 Sponsor \& Supporters. lv

\section{Session 1A: Bias and counterfactual learning 1}

- Deconfounded Video Moment Retrieval with Causal Intervention 1 Xun Yang (Sea-NExT foint Lab \& National University of Singapore), Fuli Feng (Sea-NExT Joint Lab \& National University of Singapore), Wei Ji (Sea-NExT Joint Lab \& National University of Singapore), Meng Wang (Hefei University of Technology), Tat-Seng Chua (Sea-NExT foint Lab \& National University of Singapore)

- Causal Intervention for Leveraging Popularity Bias in Recommendation

Yang Zhang (University of Science and Technology of China),

Fuli Feng (National University of Singapore), Xiangnan He (University of Science and Technology of China),

Tianxin Wei (University of Science and Technology of China),

Chonggang Song (Tencent Inc.), Guohui Ling (Tencent Inc.),

Yongdong Zhang (University of Science and Technology of China)

- AutoDebias: Learning to Debias for Recommendation 21 Jiawei Chen (University of Science and Technology of China), Hande Dong (University of Science and Technology of China), Yang Qiu (University of Science and Technology of China), Xiangnan He (University of Science and Technology of China), Xin Xin (University of Glasgow), Liang Chen (Sun Yat-Sen University), Guli Lin (Alibaba Group), Keping Yang (Alibaba Group)

- Mitigating Sentiment Bias for Recommender Systems 31 Chen Lin (Xiamen University), Xinyi Liu (Xiamen University), Guipeng Xv (Xiamen University), Hui Li (Xiamen University)

- Counterfactual Reward Modification for Streaming Recommendation with Delayed Feedback.

Xiao Zhang (Renmin University of China \& Beijing Key Laboratory of Big Data Management and Analysis Methods),

Haonan Jia (Beijing Key Laboratory of Big Data Management and Analysis Methods \& Renmin University of China), Hanjing Su (Tencent Inc.), Wenhan Wang (Tencent Inc.),

Jun Xu (Renmin University of China \& Beijing Key Laboratory of Big Data Management and Analysis Methods), Ji-Rong Wen (Renmin University of China \& Beijing Key Laboratory of Big Data Management and Analysis Methods)

\section{Session 1B: Recommendation 1}

- Joint Knowledge Pruning and Recurrent Graph Convolution for News Recommendation .......51 Yu Tian (Wuhan University), Yuhao Yang (Wuhan University),

Xudong Ren (Wuhan University), Pengfei Wang (Beijing University of Posts and Telecommunications),

Fangzhao Wu (Microsoft Research Asia), Qian Wang (Wuhan University),

Chenliang Li (Wuhan University)

- Personalized News Recommendation with Knowledge-aware Interactive Matching 61 Tao Qi (Tsinghua University), Fangzhao Wu (Microsoft Research Asia), Chuhan $\mathrm{Wu}$ (Tsinghua University), Yongfeng Huang (Tsinghua University)

- Enhanced Graph Learning for Collaborative Filtering via Mutual Information Maximization

Yonghui Yang (Hefei University of Technology),

Le $\mathrm{Wu}$ (Hefei University of Technology \& Hefei Comprehensive National Science Center), Richang Hong (Hefei University of Technology), Kun Zhang (Hefei University of Technology), Meng Wang (Hefei University of Technology \& Hefei Comprehensive National Science Center)

- ReXPlug: Explainable Recommendation using Plug-and-Play Language Model 81 Deepesh V. Hada (Indian Institute of Science, Bangalore), Vijaikumar M. (Indian Institute of Science, Bangalore), Shirish K. Shevade (Indian Institute of Science, Bangalore) 


\section{Session 1C: Searching and Ranking}

- Group based Personalized Search by Integrating Search Behaviour and Friend Network........ 92 Yujia Zhou (Renmin University of China \& Tencent), Zhicheng Dou (Renmin University of China), Bingzheng Wei (Tencent), Ruobing Xie (Tencent), Ji-Rong Wen (Renmin University of China \& MOE)

- An Image is Worth a Thousand Terms? Analysis of Visual E-Commerce Search. 102 Arnon Dagan (eBay Research), Ido Guy (eBay Research \& Ben-Gurion University of the Negev), Slava Novgorodov (eBay Research)

- Efficiently Teaching an Effective Dense Retriever with Balanced Topic Aware Sampling...... 113 Sebastian Hofstätter (TU Wien), Sheng-Chieh Lin (University of Waterloo), Jheng-Hong Yang (University of Waterloo), Jimmy Lin (University of Waterloo), Allan Hanbury (TU Wien)

- Learning a Fine-Grained Review-based Transformer Model for Personalized Product Search.

Keping Bi (University of Massachusetts, Amherst), Qingyao Ai (University of Utah),

W. Bruce Croft (University of Massachusetts, Amherst)

\section{Session 1D: Social Aspects}

- DepressionNet: A Novel Summarization Boosted Deep Framework for Depression Detection on Social Media.

Hamad Zogan (University of Technology Sydney \& College of Computer Science and Information Technology), Imran Razzak (Deakin University), Shoaib Jameel (University of Essex), Guandong Xu (University of Technology Sydney)

- Look Before You Leap: Confirming Edge Signs in Random Walk with Restart for Personalized Node Ranking in Signed Networks.

Wonchang Lee (Hanyang University), Yeon-Chang Lee (Hanyang University),

Dongwon Lee (The Pennsylvania State University), Sang-Wook Kim (Hanyang University)

- Hierarchical Multi-modal Contextual Attention Network for Fake News Detection

Shengsheng Qian (National Lab of Pattern Recognition, Institute of Automation, CAS; University of Chinese Academy of Sciences),

Jinguang Wang (HeFei University of Technology),

Jun $\mathrm{Hu}$ (National Lab of Pattern Recognition, Institute of Automation, CAS),

Quan Fang (National Lab of Pattern Recognition, Institute of Automation, CAS; University of Chinese Academy of

Sciences), Changsheng Xu (National Lab of Pattern Recognition, Institute of Automation,CAS;University of Chinese Academy of Sciences;Peng Cheng Laboratory)

- DyDiff-VAE: A Dynamic Variational Framework for Information Diffusion Prediction 163

Ruijie Wang (University of Illinois at Urbana Champaign), Zijie Huang (University of California, Los Angeles),

Shengzhong Liu (University of Illinois at Urbana Champaign),

Huajie Shao (University of Illinois at Urbana Champaign),

Dongxin Liu (University of Illinois at Urbana Champaign),

Jinyang Li (University of Illinois at Urbana Champaign),

Tianshi Wang (University of Illinois at Urbana Champaign),

Dachun Sun (University of Illinois at Urbana Champaign), Shuochao Yao (George Mason University),

Tarek Abdelzaher (University of Illinois at Urbana Champaign)

\section{Session 1E: Knowledge Structures}

- Tracing Knowledge State with Individual Cognition and Acquisition Estimation 173

Ting Long (Shanghai fiao Tong University), Yunfei Liu (Shanghai fiao Tong University), Jian Shen (Shanghai fiao Tong University), Weinan Zhang (Shanghai fiao Tong University), Yong Yu (Shanghai fiao Tong University)

- Knowledge-based Review Generation by Coherence Enhanced Text Planning..... Junyi Li (Renmin University of China), Wayne Xin Zhao (Renmin University of China), Zhicheng Wei (Huawei Cloud), Nicholas Jing Yuan (Huawei Cloud), Ji-Rong Wen (Renmin University of China)

- UGRec: Modeling Directed and Undirected Relations for Recommendation 193 Xinxiao Zhao (Shandong University), Zhiyong Cheng (Shandong Artificial Intelligence Institute, Qilu University of Technology), Lei Zhu (Shandong Normal University), Jiecai Zheng (Shandong Sport University), Xueqing Li (Shandong University) 
- DEKR: Description Enhanced Knowledge Graph for Machine Learning Method

Recommendation.

Xianshuai Cao (Shandong University), Yuliang Shi (Shandong University \& Dareway Software Co., Ltd),

Han Yu (Nanyang Technological University), Jihu Wang (Shandong University),

Xinjun Wang (Shandong University \& Dareway Software Co., Ltd),

Zhongmin Yan (Shandong University), Zhiyong Chen (Shandong University)

- Relational Learning with Gated and Attentive Neighbor Aggregator for Few-Shot Knowledge Graph Completion

Guanglin Niu (Alibaba Group), Yang Li (Alibaba Group), Chengguang Tang (Alibaba Group),

Ruiying Geng (Alibaba Group), Jian Dai (Alibaba Group), Qiao Liu (Individual),

Hao Wang (Alibaba Group), Jian Sun (Alibaba Group), Fei Huang (Alibaba Group),

Luo Si (Alibaba Group)

\section{Session 1F: Applications 1}

- AdsGNN: Behavior-Graph Augmented Relevance Modeling in Sponsored Search

Chaozhuo Li (Microsoft Research Asia), Bochen Pang (Microsoft), Yuming Liu (Microsoft),

Hao Sun (Microsoft), Zheng Liu (Microsoft Research Asia), Xing Xie (Microsoft Research Asia),

Tianqi Yang (Microsoft), Yanling Cui (Microsoft), Liangjie Zhang (Microsoft), Qi Zhang (Microsoft)

- Hybrid Learning to Rank for Financial Event Ranking.

Fuli Feng (Sea-NExT Joint Lab \& National University of Singapore),

Moxin Li (National University of Singapore), Cheng Luo (MegaTech.AI),

Ritchie Ng (National University of Singapore), Tat-Seng Chua (National University of Singapore)

- Hybrid Fusion with Intra- and Cross-Modality Attention for Image-Recipe Retrieval

Jiao Li (University of Electronic Science and Technology of China),

Xing Xu (University of Electronic Science and Technology of China),

Wei Yu (University of Electronic Science and Technology of China),

Fumin Shen (University of Electronic Science and Technology of China),

Zuo Cao (MEITUAN), Kai Zuo (MEITUAN),

Heng Tao Shen (University of Electronic Science and Technology of China)

- PreSizE: Predicting Size in E-Commerce using Transformers .

Yotam Eshel (eBay Research), Or Levi (eBay Research), Haggai Roitman (eBay Research),

Alex Nus (eBay Research)

- Understanding and Mitigating Bias in Online Health Search.

Anat Hashavit (Bar-Ilan University), Hongning Wang (University of Virginia),

Raz Lin (Bar-Ilan University), Tamar Stern (Bar-Ilan University), Sarit Kraus (Bar-Ilan University)

\section{Session 2A: Bias and Counterfactual Learning 2}

- Enhanced Doubly Robust Learning for Debiasing Post-Click Conversion Rate Estimation ... 275 Siyuan Guo (filin University), Lixin Zou (Baidu Inc.), Yiding Liu (Baidu Inc.), Wenwen Ye (Baidu Inc.),

Suqi Cheng (Baidu Inc.), Shuaiqiang Wang (Baidu Inc.), Hechang Chen (filin University),

Dawei Yin (Baidu Inc.), Yi Chang (Filin University)

- Adapting Interactional Observation Embedding for Counterfactual Learning to Rank 285 Mouxiang Chen (Zhejiang University \& Alibaba-Zhejiang University foint Institute of Frontier Technologies), Chenghao Liu (Salesforce Research Asia),

Jianling Sun (Zhejiang University \& Alibaba-Zhejiang University foint Institute of Frontier Technologies), Steven C.H. Hoi (Salesforce Research Asia)

- This Is Not What We Ordered: Exploring Why Biased Search Result Rankings Affect User Attitudes on Debated Topics 295

Tim Draws (Delft University of Technology), Nava Tintarev (Maastricht University),

Ujwal Gadiraju (Delft University of Technology), Alessandro Bozzon (Delft University of Technology),

Benjamin Timmermans (IBM)

- Societal Biases in Retrieved Contents: Measurement Framework and Adversarial Mitigation of BERT Rankers. 306

Navid Rekabsaz (fohannes Kepler University), Simone Kopeinik (Know-Center GmbH),

Markus Schedl (fohannes Kepler University) 


\section{Session 2B: Recommendation 2}

- Bootstrapping User and Item Representations for One-Class Collaborative Filtering

Dongha Lee (Pohang University of Science and Technology (POSTECH)),

SeongKu Kang (Pohang University of Science and Technology (POSTECH)),

Hyunjun Ju (Pohang University of Science and Technology (POSTECH)),

Chanyoung Park (Korea Advanced Institute of Science and Technology (KAIST)),

Hwanjo Yu (Pohang University of Science and Technology (POSTECH))

- Unsupervised Proxy Selection for Session-based Recommender Systems

Junsu Cho (Pohang University of \& Technology), SeongKu Kang (Pohang University of Science \&Technology),

Dongmin Hyun (Pohang University of Science \& Technology),

Hwanjo Yu (Pohang University of Science \& Technology)

- xLightFM: Extremely Memory-Efficient Factorization Machine

Gangwei Jiang (University of Science and Technology of China),

Hao Wang (University of Science and Technology of China),

Jin Chen (University of Electronic Science and Technology of China), Haoyu Wang (SUNY Buffalo),

Defu Lian (University of Science and Technology of China),

Enhong Chen (University of Science and Technology of China)

\section{Session 2C: Sequences and Sessions}

- Counterfactual Data-Augmented Sequential Recommendation

Zhenlei Wang (Renmin University of China), Jingsen Zhang (Renmin University of China),

Hongteng Xu (Renmin University of China), Xu Chen (Renmin University of China),

Yongfeng Zhang (Rutgers University), Wayne Xin Zhao (Renmin University of China),

Ji-Rong Wen (Renmin University of China)

- StackRec: Efficient Training of Very Deep Sequential Recommender Models

by Iterative Stacking.

Jiachun Wang (South China University of Technology \& Chinese Academy of Sciences),

Fajie Yuan (Westlake University \& Tencent), Jian Chen (South China University of Technology),

Qingyao Wu (South China University of Technology), Min Yang (Chinese Academy of Sciences),

Yang Sun (Chinese Academy of Sciences), Guoxiao Zhang (Tencent)

- Cause Rec: Counterfactual User Sequence Synthesis for Sequential Recommendation. 367

Shengyu Zhang (Zhejiang University), Dong Yao (Zhejiang University), Zhou Zhao (Zhejiang University), Tat-Seng Chua (National University of Singapore), Fei Wu (Zhejiang University)

- Sequential Recommendation with Graph Neural Networks 378

Jianxin Chang (Tsinghua University), Chen Gao (Tsinghua University), Yu Zheng (Tsinghua University),

Yiqun Hui (Beijing Kuaishou Technology Co., Ltd.), Yanan Niu (Beijing Kuaishou Technology Co., Ltd.),

Yang Song (Beijing Kuaishou Technology Co., Ltd.), Depeng Jin (Tsinghua University),

Yong Li (Tsinghua University)

- Category-aware Collaborative Sequential Recommendation

Renqin Cai (University of Virginia), Jibang Wu (University of Virginia), Aidan San (University of Virginia),

Chong Wang (Bytedance), Hongning Wang (University of Virginia)

\section{Session 2D: Time Matters}

- Event Occurrence Date Estimation based on Multivariate Time Series Analysis over Temporal Document Collections.

Jiexin Wang (Kyoto University), Adam Jatowt (University of Innsbruck),

Masatoshi Yoshikawa (Kyoto University)

- Temporal Knowledge Graph Reasoning Based on Evolutional Representation Learning... 408 Zixuan Li (University of Chinese Academy of Sciences \& Chinese Academy of Science),

Xiaolong Jin (University of Chinese Academy of Sciences \& Chinese Academy of Science), Wei Li (Baidu Inc.),

Saiping Guan (University of Chinese Academy of Sciences \& Chinese Academy of Science),

Jiafeng Guo (University of Chinese Academy of Sciences \& Chinese Academy of Science),

Huawei Shen (University of Chinese Academy of Sciences \& Chinese Academy of Science),

Yuanzhuo Wang (University of Chinese Academy of Sciences \& Chinese Academy of Science),

Xueqi Cheng (University of Chinese Academy of Sciences \& Chinese Academy of Science) 
- Summarize Dates First: A Paradigm Shift in Timeline Summarization

Moreno La Quatra (Politecnico di Torino), Luca Cagliero (Politecnico di Torino),

Elena Baralis (Politecnico di Torino),

Alberto Messina (Radiotelevisione Italiana (RAI), Centre for Research and Technological Innovation),

Maurizio Montagnuolo (Radiotelevisione Italiana (RAI), Centre for Research and Technological Innovation)

- TIE: A Framework for Embedding-based Incremental Temporal Knowledge Graph

Completion

Jiapeng Wu (McGill University \& MILA), Yishi Xu (University of Montreal \& MILA),

Yingxue Zhang (Montreal Research Center \& Huawei Noah's Ark Lab), Chen Ma (McGill University),

Mark Coates (McGill University), Jackie Chi Kit Cheung (McGill University \& MILA)

- Allowing for The Grounded Use of Temporal Difference Learning in Large Ranking

Models via Substate Updates

Daniel Cohen (Brown University)

\section{Session 2E: Question Answering}

- Answer Complex Questions: Path Ranker Is All You Need

Xinyu Zhang (Huawei), Ke Zhan (Huawei), Enrui Hu (Huawei), Chengzhen Fu (Huawei),

Lan Luo (Huawei), Hao Jiang (Huawei), Yantao Jia (Huawei), Fan Yu (Huawei),

Zhicheng Dou (Renmin University of China), Zhao Cao (Huawei),

Lei Chen (Hong Kong University of Science and Technology)

- Reinforcement Learning from Reformulations in Conversational Question

Answering over Knowledge Graphs

Magdalena Kaiser (Max Planck Institute for Informatics), Rishiraj Saha Roy (Max Planck Institute for Informatics),

Gerhard Weikum (Max Planck Institute for Informatics)

- Ranking User-Generated Content via Multi-Relational Graph Convolution ..

Kanika Narang (University of Illinois at Urbana-Champaign),

Adit Krishnan (University of Illinois at Urbana-Champaign),

Junting Wang (University of Illinois at Urbana-Champaign),

Chaoqi Yang (University of Illinois at Urbana-Champaign),

Hari Sundaram (University of Illinois at Urbana-Champaign),

Carolyn Sutter (University of Illinois at Urbana-Champaign)

- Answering Any-hop Open-domain Questions with Iterative Document Reranking

Yuyu Zhang (Georgia Institute of Technology), Ping Nie (Peking University),

Arun Ramamurthy (Siemens Corporate Technology), Le Song (Georgia Institute of Technology)

- Multimodal Activation: Awakening Dialog Robots without Wake Words

Liqiang Nie (Shandong University), Mengzhao Jia (Shandong University),

Xuemeng Song (Shandong University), Ganglu Wu (Alibaba Group),

Harry Cheng (Shandong University), Jian Gu (Alibaba Group)

\section{Session 2F: Applications 2}

- RCD: Relation Map Driven Cognitive Diagnosis for Intelligent Education Systems

Weibo Gao (University of Science and Technology of China), Qi Liu (University of Science and Technology of China),

Zhenya Huang (University of Science and Technology of China),

Yu Yin (University of Science and Technology of China),

Haoyang Bi (University of Science and Technology of China),

$\mathrm{Mu}$-Chun Wang (University of Science and Technology of China),

Jianhui Ma (University of Science and Technology of China), Shijin Wang (IFLYTEK), Yu Su (IFLYTEK)

- Self-Supervised Contrastive Learning for Code Retrieval and Summarization

via Semantic-Preserving Transformations ...

Nghi D. Q. Bui (Huawei Research Center, Ireland), Yijun Yu (Huawei Research Center, Ireland),

Lingxiao Jiang (Singapore Management University)

\section{Session 3A: Conversational IR 1}

- Initiative-Aware Self-Supervised Learning for Knowledge-Grounded Conversations 522 Chuan Meng (Shandong University), Pengjie Ren (Shandong University), Zhumin Chen (Shandong University),

Zhaochun Ren (Shandong University), Tengxiao Xi (Shandong University),

Maarten de Rijke (University of Amsterdam \& Ahold Delhaize Research) 
- Wizard of Search Engine: Access to Information Through Conversations with Search Engines..

Pengjie Ren (Shandong University), Zhongkun Liu (Shandong University),

Xiaomeng Song (Shandong University), Hongtao Tian (Shandong University),

Zhumin Chen (Shandong University), Zhaochun Ren (Shandong University),

Maarten de Rijke (University of Amsterdam \& Ahold Delhaize)

- Semi-Supervised Variational Reasoning for Medical Dialogue Generation

Dongdong Li (Shandong University), Zhaochun Ren (Shandong University),

Pengjie Ren (Shandong University), Zhumin Chen (Shandong University),

Miao Fan (Baidu Inc.), Jun Ma (Shandong University),

Maarten de Rijke (University of Amsterdam \& Ahold Delhaize)

- One Chatbot Per Person: Creating Personalized Chatbots based on Implicit User Profiles .... 555

Zhengyi Ma (Renmin University of China \& Beijing Key Laboratory of Big Data Management and Analysis Methods),

Zhicheng Dou (Renmin University of China \& Beijing Key Laboratory of Big Data Management and Analysis

Methods),

Yutao Zhu (Université de Montréal), Hanxun Zhong (Renmin University of China \& Beijing Key Laboratory of Big

Data Management and Analysis Methods),

Ji-Rong Wen (Renmin University of China \& Beijing Key Laboratory of Big Data Management and Analysis Methods)

- Partner Matters! An Empirical Study on Fusing Personas for Personalized Response

Selection in Retrieval-Based Chatbots

Jia-Chen Gu (University of Science and Technology of China), Hui Liu (Queen's University),

Zhen-Hua Ling (University of Science and Technology of China),

Quan Liu (University of Science and Technology of China \& iFLYTEK Research),

Zhigang Chen (iFLYTEK Research), Xiaodan Zhu (Queen's University)

\section{Session 3B: Recommendation 3}

- Learning Recommender Systems with Implicit Feedback via Soft Target Enhancement 575

Mingyue Cheng (University of Science and Technology of China),

Fajie Yuan (Westlake University \& Tencent Inc.), Qi Liu (University of Science and Technology of China),

Shenyang Ge (FD Inc), Zhi Li (University of Science and Technology of China),

Runlong Yu (University of Science and Technology of China),

Defu Lian (University of Science and Technology of China),

Senchao Yuan (University of Science and Technology of China),

Enhong Chen (University of Science and Technology of China)

- Set2setRank: Collaborative Set to Set Ranking for Implicit Feedback based

Recommendation .....

Lei Chen (Hefei University of Technology),

Le Wu (Hefei University of Technology \& Hefei Comprehensive National Science Center),

Kun Zhang (Hefei University of Technology), Richang Hong (Hefei University of Technology),

Meng Wang (Hefei University of Technology \& Hefei Comprehensive National Science Center)

- Package Recommendation with Intra- and Inter-Package Attention Networks

Chen Li (Beijing University of Posts and Telecommunications \& Tencent),Yuanfu Lu (Tencent),

Wei Wang (Tencent), Chuan Shi (Beijing University of Posts and Telecommunications),

Ruobing Xie (Tencent), Haili Yang (Tencent), Cheng Yang (Beijing University of Posts and Telecommunications),

Xu Zhang (Tencent), Leyu Lin (Tencent)

- A Guided Learning Approach for Item Recommendation via Surrogate Loss Learning 605

Ahmed Rashed (University of Hildesheim), Josif Grabocka (University of Freiburg),

Lars Schmidt-Thieme (University of Hildesheim)

- Structured Graph Convolutional Networks with Stochastic Masks for Recommender Systems

Huiyuan Chen (Visa Research), Lan Wang (Visa Research), Yusan Lin (Visa Research),

Chin-Chia Michael Yeh (Visa Research), Fei Wang (Visa Research), Hao Yang (Visa Research) 


\section{Session 3C: Neural IR}

- WGCN: Graph Convolutional Networks with Weighted Structural Features

Yunxiang Zhao (The University of Melbourne), Jianzhong Qi (The University of Melbourne),

Qingwei Liu (The University of Melbourne), Rui Zhang (www.ruizhang.info)

- Privacy Protection in Deep Multi-modal Retrieval

Peng-Fei Zhang (The University of Queensland), Yang Li (The University of Queensland),

Zi Huang (The University of Queensland), Hongzhi Yin (The University of Queensland)

- Learning Discriminative Neural Representations for Event Detection Jinzhi Liao (National University of Defense Technology), Xiang Zhao (National University of Defense Technology), Xinyi Li (National University of Defense Technology), Lingling Zhang (Xi'an fiaotong University),

Jiuyang Tang (National University of Defense Technology)

- Not All Relevance Scores are Equal: Efficient Uncertainty and Calibration Modeling for Deep Retrieval Models.

Daniel Cohen (Brown University), Bhaskar Mitra (Microsoft), Oleg Lesota (fohannes Kepler University),

Navid Rekabsaz (fohannes Kepler University), Carsten Eickhoff (Brown University)

- Interpretable Graph Similarity Computation via Differentiable Optimal Alignment of Node Embeddings.

Khoa D. Doan (Virginia Tech), Saurav Manchanda (University of Minnesota),

Suchismit Mahapatra (University of Buffalo), Chandan K. Reddy (Virginia Tech)

\section{Session 3D: Cross-domain IR}

- MMConv: An Environment for Multimodal Conversational Search across Multiple

Domains

Lizi Liao (Sea-NExT foint Lab \& National University of Singapore),

Le Hong Long (National University of Singapore), Zheng Zhang (Tsinghua University),

Minlie Huang (Tsinghua University), Tat-Seng Chua (National University of Singapore)

- Video Corpus Moment Retrieval with Contrastive Learning.

Hao Zhang (Nanyang Technological University), Aixin Sun (Nanyang Technological University),

Wei Jing (Agency for Science, Technology and Research),

Guoshun Nan (Singapore University of Technology and Design),

Liangli Zhen (Agency for Science, Technology and Research),

Joey Tianyi Zhou (Agency for Science, Technology and Research),

Rick Siow Mong Goh (Agency for Science, Technology and Research)

- One Person, One Model, One World: Learning Continual User Representation without Forgetting.

Fajie Yuan (Westlake University \& Tencent), Guoxiao Zhang (Tencent), Alexandros Karatzoglou (Google),

Joemon Jose (University of Glasgow), Beibei Kong (Tencent), Yudong Li (Tencent)

- Learning Domain Semantics and Cross-Domain Correlations for Paper Recommendation ... 706 Yi Xie (Shandong University), Yuqing Sun (Shandong University), Elisa Bertino (Purdue University)

- FedCT: Federated Collaborative Transfer for Recommendation 716

Shuchang Liu (Rutgers University), Shuyuan Xu (Rutgers University), Wenhui Yu (Alibaba Group),

Zuohui Fu (Rutgers University), Yongfeng Zhang (Rutgers University),

Amelie Marian (Rutgers University

\section{Session 3E: Diversity and Novelty}

- Self-supervised Graph Learning for Recommendation

Jiancan Wu (University of Science and Technology of China), Xiang Wang (National University of Singapore),

Fuli Feng (National University of Singapore), Xiangnan He (University of Science and Technology of China),

Liang Chen (Sun Yat-sen University), Jianxun Lian (Microsoft Research Asia),

Xing Xie (Microsoft Research Asia)

- Modeling Intent Graph for Search Result Diversification 736

Zhan Su (Renmin University of China), Zhicheng Dou (Renmin University of China),

Yutao Zhu (Université de Montréal), Xubo Qin (Renmin University of China),

Ji-Rong Wen (Beijing Key Laboratory of Big Data Management and Analysis Methods \& Key Laboratory of Data

Engineering and Knowledge Engineering) 
- Enhancing Domain-Level and User-Level Adaptivity in Diversified Recommendation.

Yile Liang (Wuhan University), Tieyun Qian (Wuhan University),

Qing Li (Hong Kong Polytechnic University), Hongzhi Yin (The University of Queensland)

- Graph Meta Network for Multi-Behavior Recommendation.

Lianghao Xia (South China University of Technology), Yong Xu (South China University of Technology),

Chao Huang (fD Finance America Corporation), Peng Dai (fD Finance America Corporation),

Liefeng Bo (JD Finance America Corporation)

- Fairness among New Items in Cold Start Recommender Systems

Ziwei Zhu (Texas A\&M University), Jingu Kim (Netflix), Trung Nguyen (Netflix),

Aish Fenton (Netflix), James Caverlee (Texas A\&M University)

\section{Session 3F: Applications 3}

- Make It Easy: An Effective End-to-End Entity Alignment Framework

Congcong Ge (Zhejiang University), Xiaoze Liu (Zhejiang University), Lu Chen (Zhejiang University),

Baihua Zheng (Singapore Management University),

Yunjun Gao (Zhejiang University \& Alibaba-Zhejiang University foint Institute of Frontier Technologies)

- Files of a Feather Flock Together? Measuring and Modeling How Users Perceive File Similarity in Cloud Storage.

Will Brackenbury (University of Chicago), Galen Harrison (University of Chicago),

Kyle Chard (University of Chicago), Aaron Elmore (University of Chicago),

Blase Ur (University of Chicago)

- CINES: Explore Citation Network and Event Sequences for Citation Forecasting 798

Fang He (Pennsylvania State University), Wang-Chien Lee (Pennsylvania State University),

Tao-Yang Fu (Pennsylvania State University), Zhen Lei (Pennsylvania State University)

\section{Session 4A: Conversational IR 2}

- Learning to Ask Appropriate Questions in Conversational Recommendation 808

Xuhui Ren (The University of Queensland), Hongzhi Yin (The University of Queensland),

Tong Chen (The University of Queensland), Hao Wang (Alibaba Group),

Zi Huang (The University of Queensland),

Kai Zheng (University of Electronic Science and Technology of China)

- Multi-Modal Supplementary-Complementary Summarization using Multi-Objective Optimization

Anubhav Jangra (Indian Institute of Technology Patna),

Sriparna Saha (Indian Institute of Technology Patna), Adam Jatowt (University of Innsbruck), Mohammed

Hasanuzzaman (Munster Technological University)

- Few-Shot Conversational Dense Retrieval

Shi Yu (Tsinghua University), Zhenghao Liu (Tsinghua University), Chenyan Xiong (Microsoft Research),

Tao Feng (Tsinghua University), Zhiyuan Liu (Tsinghua University)

- Conversational Fashion Image Retrieval via Multiturn Natural Language Feedback

Yifei Yuan (The Chinese University of Hong Kong), Wai Lam (The Chinese University of Hong Kong)

\section{Session 4B: Recommendation 4}

- Neural Graph Matching based Collaborative Filtering 849

Yixin Su (University of Melbourne), Rui Zhang (www.ruizhang.info),

Sarah M. Erfani (University of Melbourne), Junhao Gan (University of Melbourne)

- The World is Binary: Contrastive Learning for Denoising Next Basket Recommendation..... 859

Yuqi Qin (Beijing University of Posts and Telecommunications),

Pengfei Wang (Beijing University of Posts and Telecommunications),

Chenliang Li (School of Cyber Science and Engineering, Wuhan University)

- Dual Attention Transfer in Session-based Recommendation with Multi-dimensional Integration 869

Chen Chen (Xidian University), Jie Guo (Xidian University), Bin Song (Xidian University) 
- User-Centric Path Reasoning towards Explainable Recommendation

Chang-You Tai (Academia Sinica), Liang-Ying Huang (Academia Sinica),

Chien-Kun Huang (Academia Sinica), Lun-Wei Ku (Academia Sinica)

- On Interpretation and Measurement of Soft Attributes for Recommendation 890 Krisztian Balog (University of Stavanger), Filip Radlinski (Google), Alexandros Karatzoglou (Google)

\section{Session 4C: Learning to Rank}

- How do Online Learning to Rank Methods Adapt to Changes of Intent?

Shengyao Zhuang (The University of Queensland), Guido Zuccon (The University of Queensland)

- Scalable Personalised Item Ranking through Parametric Density Estimation Riku Togashi (CyberAgent, Inc. \& Waseda University), Masahiro Kato (CyberAgent, Inc.), Mayu Otani (CyberAgent, Inc.), Tetsuya Sakai (Waseda University), Shin'ichi Satoh (CyberAgent, Inc.)

- New Insights into Metric Optimization for Ranking-based Recommendation . 932 Roger Zhe Li (Delft University of Technology), Julián Urbano (Delft University of Technology), Alan Hanjalic (Delft University of Technology)

- Fast Attention-based Learning-To-Rank Model for Structured Map Search 942 Chiqun Zhang (Microsoft), Michael R. Evans (Microsoft), Max Lepikhin (Microsoft), Dragomir Yankov (Microsoft)

- Learning to Rank for Mathematical Formula Retrieval 952 Behrooz Mansouri (Rochester Institute of Technology), Richard Zanibbi (Rochester Institute of Technology), Douglas W. Oard (University of Maryland)

\section{Session 4D: Legal IR}

- Investigating User Behavior in Legal Case Retrieval 962 Yunqiu Shao (BNRist, DCST, Tsinghua University), Yueyue Wu (BNRist, DCST, Tsinghua University), Yiqun Liu (BNRist, DCST, Tsinghua University), Jiaxin Mao (GSAI, Renmin University of China), Min Zhang (BNRist, DCST, Tsinghua University), Shaoping Ma (BNRist, DCST, Tsinghua University)

- NeurJudge: A Circumstance-aware Neural Framework for Legal Judgment Prediction 973 Linan Yue (University of Science and Technology of China), Qi Liu (University of Science and Technology of China), Binbin Jin (University of Science and Technology of China), Han Wu (University of Science and Technology of China), Kai Zhang (University of Science and Technology of China), Yanqing An (Univ. of Science and Technology of China), Mingyue Cheng (University of Science and Technology of China), Biao Yin (University of Science and Technology of China), Dayong Wu (IFLYTEK)

- Legal Judgment Prediction via Relational Learning 983 Qian Dong (Institute of Software, Chinese Academy of Sciences \& University of Chinese Academy of Sciences), Shuzi Niu (Institute of Software, Chinese Academy of Sciences)

- Legal Judgment Prediction with Multi-Stage Case Representation Learning in the Real Court Setting

Luyao Ma (Peking University \& Alibaba Group), Yating Zhang (Alibaba Group),

Tianyi Wang (Alibaba Group), Xiaozhong Liu (Indiana University Bloomington),

Wei Ye (Peking University), Changlong Sun (Alibaba Group), Shikun Zhang (Peking University)

- Cross-Domain Contract Element Extraction with a Bi-directional Feedback Clause-Element Relation Network

Zihan Wang (Shandong University), Hongye Song (Alibaba Group), Zhaochun Ren (Shandong University),

Pengjie Ren (Shandong University), Zhumin Chen (Shandong University),

Xiaozhong Liu (Indiana University Bloomington), Hongsong Li (Alibaba Group),

Maarten de Rijke (University of Amsterdam \& Ahold Delhaize Research)

\section{Session 4E: Fairness}

- TFROM: A Two-sided Fairness-Aware Recommendation Model for Both Customers and Providers

Yao Wu (Shanghai fiao Tong University), Jian Cao (Shanghai fiao Tong University),

Guandong Xu (University of Technology Sydney), Yudong Tan (Ctrip.com International Ltd Shanghai) 
- Computationally Efficient Optimization of Plackett-Luce Ranking Models for Relevance and Fairness

Harrie Oosterhuis (Radboud University)

- When Fair Ranking Meets Uncertain Inference

Avijit Ghosh (Northeastern University), Ritam Dutt (Carnegie Mellon University),

Christo Wilson (Northeastern University)

- Policy-Gradient Training of Fair and Unbiased Ranking Functions

Himank Yadav (Cornell University), Zhengxiao Du (Tsinghua University), Thorsten Joachims (Cornell University)

- Towards Personalized Fairness based on Causal Notion

Yunqi Li (Rutgers University), Hanxiong Chen (Rutgers University), Shuyuan Xu (Rutgers University),

Yingqiang Ge (Rutgers University), Yongfeng Zhang (Rutgers University)

\section{Session 4F: Adversarial IR}

- DAIR: A Query-Efficient Decision-based Attack on Image Retrieval Systems

Mingyang Chen (University of New South Wales), Junda Lu (University of New South Wales),

Yi Wang (Dongguan University of Technology),

Jianbin Qin (Shenzhen Institute of Computing Sciences, Shenzhen University),

Wei Wang (University of New South Wales)

- Fight Fire with Fire: Towards Robust Recommender Systems via Adversarial Poisoning Training

Chenwang Wu (University of Science and Technology of China),

Defu Lian (University of Science and Technology of China), Yong Ge (University of Arizona),

Zhihao Zhu (University of Science and Technology of China),

Enhong Chen (University of Science and Technology of China),

Senchao Yuan (University of Science and Technology of China)

- Adversarial-Enhanced Hybrid Graph Network for User Identity Linkage

Xiaolin Chen (Shandong University), Xuemeng Song (Shandong University),

Guozhen Peng (Shandong University), Shanshan Feng (Harbin Institute of Technology),

Liqiang Nie (Shandong University)

- A Study of Defensive Methods to Protect Visual Recommendation Against Adversarial Manipulation of Images.

Vito Walter Anelli (Polytechnic University of Bari), Yashar Deldjoo (Polytechnic University of Bari),

Tommaso Di Noia (Polytechnic University of Bari), Daniele Malitesta (Polytechnic University of Bari),

Felice Antonio Merra (Polytechnic University of Bari)

\section{Session 5A: Multi-modal IR}

- Dynamic Modality Interaction Modeling for Image-Text Retrieval.

Leigang Qu (Shandong University), Meng Liu (Shandong fianzhu University),

Jianlong Wu (Shandong University), Zan Gao (Shandong Artificial Intelligence Institute),

Liqiang Nie (Shandong University)

- Hierarchical Cross-Modal Graph Consistency Learning for Video-Text Retrieval

Weike Jin (Zhejiang University), Zhou Zhao (Zhejiang University),

Pengcheng Zhang (Zhejiang University), Jieming Zhu (Huawei Noah’s Ark Lab),

Xiuqiang He (Huawei Noah's Ark Lab), Yueting Zhuang (Zhejiang University)

- PAN: Prototype-based Adaptive Network for Robust Cross-modal Retrieval 1125

Zhixiong Zeng (Institute of Automation, Chinese Academy of Sciences \& University of Chinese Academy of Sciences),

Shuai Wang (Institute of Automation, Chinese Academy of Sciences \& University of Chinese Academy of Sciences), $\mathrm{Nan} \mathrm{Xu}$ (Institute of Automation, Chinese Academy of Sciences \& University of Chinese Academy of Sciences), Wenji Mao (Institute of Automation, Chinese Academy of Sciences \& University of Chinese Academy of Sciences)

- Multi-Type Textual Reasoning for Product-Aware Answer Generation 1135 Yue Feng (Baidu Research), Zhaochun Ren (Baidu Research), Weijie Zhao (Baidu Research), Mingming Sun (Baidu Research), Ping Li (Baidu Research)

- Heterogeneous Attention Network for Effective and Efficient Cross-modal Retrieval. 1146 Tan Yu (Baidu Research), Yi Yang (Baidu Inc.), Yi Li (Baidu Inc.), Lin Liu (Baidu Inc.), Hongliang Fei (Baidu Research), Ping Li (Baidu Research) 


\section{Session 5B: Exploration and Cold Start}

- Learning Graph Meta Embeddings for Cold-Start Ads in Click-Through Rate Prediction .... 1157 Wentao Ouyang (Alibaba Group), Xiuwu Zhang (Alibaba Group), Shukui Ren (Alibaba Group), Li Li (Alibaba Group), Kun Zhang (Alibaba Group), Jinmei Luo (Alibaba Group), Zhaojie Liu (Alibaba Group), Yanlong Du (Alibaba Group)

- Learning to Warm Up Cold Item Embeddings for Cold-start Recommendation with Meta Scaling and Shifting Networks.

Yongchun Zhu (Institute of Computing Technology, Chinese Academy of Sciences, University of Chinese Academy of Sciences, \& Tencent), Ruobing Xie (Tencent), Fuzhen Zhuang (Beihang University), Kaikai Ge (Tencent), Ying Sun (Institute of Computing Technology, Chinese Academy of Sciences \& University of Chinese Academy of Sciences),

Xu Zhang (Tencent), Leyu Lin (Tencent), Juan Cao (Institute of Computing Technology, Chinese Academy of Sciences \& University of Chinese Academy of Sciences)

- FORM: Follow the Online Regularized Meta-Leader for Cold-Start Recommendation 1177

Xuehan Sun (Shanghai fiao Tong University), Tianyao Shi (Shanghai fiao Tong University),

Xiaofeng Gao (Shanghai Jiao Tong University), Yanrong Kang (Tencent Advertising and Marketing Service),

Guihai Chen (Shanghai fiao Tong University)

- Privileged Graph Distillation for Cold Start Recommendation

Shuai Wang (Hefei University of Technology), Kun Zhang (Hefei University of Technology),

Le Wu (Hefei University of Technology\& Hefei Comprehensive National Science Center),

Haiping Ma (Anhui University), Richang Hong (Hefei University of Technology),

Meng Wang (Hefei University of Technology \& Hefei Comprehensive National Science Center)

- Supporting Metacognition during Exploratory Search with the OrgBox

Anita Crescenzi (University of North Carolina at Chapel Hill),

Austin R. Ward (University of North Carolina at Chapel Hill),

Yuan Li (University of North Carolina at Chapel Hill), Rob Capra (University of North Carolina at Chapel Hill)

\section{Session 5C: Mining and Classification}

- Should Graph Convolution Trust Neighbors? A Simple Causal Inference Method 1208

Fuli Feng (Sea-NExT foint Lab \& National University of Singapore),

Weiran Huang (The Chinese University of Hong Kong),

Xiangnan He (University of Science and Technology of China), Xin Xin (University of Glasgow),

Qifan Wang (Google US), Tat-Seng Chua (National University of Singapore)

- Meta-Inductive Node Classification across Graphs

Zhihao Wen (Singapore Management University), Yuan Fang (Singapore Management University),

Zemin Liu (Singapore Management University)

- Iterative Network Pruning with Uncertainty Regularization for Lifelong Sentiment

Classification .

Binzong Geng (University of Science and Technology of China \& Shenzhen Institutes of Advanced Technology,

Chinese Academy of Sciences),

Min Yang (Shenzhen Institutes of Advanced Technology, Chinese Academy of Sciences),

Fajie Yuan (Westlake University \& Tencent),

Shupeng Wang (Shenzhen Institutes of Advanced Technology, Chinese Academy of Sciences),

Xiang Ao (Institute of Computing Technology, Chinese Academy of Sciences),

Ruifeng Xu (Harbin Institute of Technology (Shenzhen))

- Decoupling Representation Learning and Classification for GNN-based Anomaly

Detection.

Yanling Wang (Renmin University of China), Jing Zhang (Renmin University of China),

Shasha Guo (Renmin University of China), Hongzhi Yin (The University of Queensland),

Cuiping Li (Renmin University of China), Hong Chen (Renmin University of China)

- “Did you buy it already?” Detecting Users Purchase-State From

Their Product-Related Questions

Lital Kuchy (Amazon), David Carmel (Amazon), Thomas Huet (Amazon),

Elad Kravi (Amazon) 


\section{Session 5D: Click Models and Prediction}

- A Graph-Enhanced Click Model for Web Search.

Jianghao Lin (Shanghai fiao Tong University), Weiwen Liu (Huawei Noah's Ark Lab),

Xinyi Dai (Shanghai fiao Tong University), Weinan Zhang (Shanghai fiao Tong University),

Shuai Li (Shanghai fiao Tong University), Ruiming Tang (Huawei Noah's Ark Lab),

Xiuqiang He (Huawei Noah's Ark Lab), Jianye Hao (Huawei Noah's Ark Lab),

Yong Yu (Shanghai fiao Tong University)

- ScaleFreeCTR: MixCache-based Distributed Training System for CTR Models with Huge Embedding Table.

Huifeng Guo (Huawei Noah's Ark Lab), Wei Guo (Huawei Noah's Ark Lab),

Yong Gao (Huawei Noah's Ark Lab), Ruiming Tang (Huawei Noah's Ark Lab),

Xiuqiang He (Huawei Noah's Ark Lab), Wenzhi Liu (Huawei Noah's Ark Lab)

- Looking at CTR Prediction Again: Is Attention All You Need?

Yuan Cheng (Career Science Lab, BOSS Zhipin), Yanbo Xue (Career Science Lab, BOSS Zhipin)

- Clicks can be Cheating: Counterfactual Recommendation for Mitigating Clickbait Issue .... 1288

Wenjie Wang (National University of Singapore), Fuli Feng (National University of Singapore),

Xiangnan He (University of Science and Technology of China),

Hanwang Zhang (Nanyang Technological University), Tat-Seng Chua (National University of Singapore)

- A General Method For Automatic Discovery of Powerful Interactions In Click-Through Rate Prediction

Ze Meng (Tsinghua University), Jinnian Zhang (University of Wisconsin Madison),

Yumeng Li (Alibaba Group), Jiancheng Li (Alibaba Group), Tanchao Zhu (Alibaba Group),

Lifeng Sun (Ministry of Education \& Tsinghua University)

\section{Session 5E: Efficiency}

- How Powerful are Interest Diffusion on Purchasing Prediction: A Case Study of Taocode .. 1308 Xuanwen Huang (Zhejiang University), Yang Yang (Zhejiang University),

Ziqiang Cheng (Zhejiang University), Shen Fan (Alibaba Group), Zhongyao Wang (Alibaba Group), Juren Li (Zhejiang University), Jun Zhang (Alibaba Group), Jingmin Chen (Alibaba Group)

- Binary Neural Network Hashing for Image Retrieval

Wanqian Zhang (Institute of Information Engineering, Chinese Academy of Sciences \& University of Chinese

Academy of Sciences),

Dayan Wu (Institute of Information Engineering, Chinese Academy of Sciences),

Yu Zhou (Institute of Information Engineering, Chinese Academy of Sciences \& University of Chinese Academy of Sciences),

Bo Li (Institute of Information Engineering, Chinese Academy of Sciences \& University of Chinese Academy of

Sciences),

Weiping Wang (Institute of Information Engineering, Chinese Academy of Sciences \& University of Chinese Academy of Sciences),

Dan Meng (Institute of Information Engineering, Chinese Academy of Sciences \& University of Chinese Academy of Sciences)

- Long-Tail Hashing

Yong Chen (Peking University \& Pazhou Lab), Yuqing Hou (Meituan),

Shu Leng (Tsinghua University), Qing Zhang (Meituan), Zhouchen Lin (Peking University \& Pazhou Lab),

Dell Zhang (Blue Prism AI Labs \& Birkbeck, University of London)

- PTHash: Revisiting FCH Minimal Perfect Hashing

Giulio Ermanno Pibiri (ISTI-CNR), Roberto Trani (ISTI-CNR)

- Intra-Document Cascading: Learning to Select Passages for Neural Document Ranking ..... 1349 Sebastian Hofstätter (TU Wien), Bhaskar Mitra (Microsoft),

Hamed Zamani (University of Massachusetts, Amherst), Nick Craswell (Microsoft),

Allan Hanbury (TU Wien)

\section{Session 6A: Multimedia IR}

- Improving Video Retrieval by Adaptive Margin 1359 Feng He (Baidu Inc.), Qi Wang (Baidu Inc.), Zhifan Feng (Baidu Inc.), Wenbin Jiang (Baidu Inc.), Yajuan Lü (Baidu Inc.), Yong Zhu (Baidu Inc.), Xiao Tan (Baidu Inc.) 
- Comprehensive Linguistic-Visual Composition Network for Image Retrieval

Haokun Wen (Shandong University), Xuemeng Song (Shandong University),

Xin Yang (Shandong University), Yibing Zhan (FD Explore Academy),

Liqiang Nie (Shandong University)

- GilBERT: Generative Vision-Language Pre-Training for Image-Text Retrieval

Weixiang Hong (Ant Group), Kaixiang Ji (Ant Group), Jiajia Liu (Ant Group),

Jian Wang (Ant Group), Jingdong Chen (Ant Group), Wei Chu (Ant Group)

- DeepQAMVS: Query-Aware Hierarchical Pointer Networks for Multi-Video

Summarization

Safa Messaoud (University of Illinois at Urbana-Champaign), Ismini Lourentzou (Virginia Tech),

Assma Boughoula (University of Illinois at Urbana-Champaign),

Mona Zehni (University of Illinois at Urbana-Champaign),

Zhizhen Zhao (University of Illinois at Urbana-Champaign),

Chengxiang Zhai (University of Illinois at Urbana-Champaign),

Alexander G. Schwing (University of Illinois at Urbana-Champaign)

\section{Session 6B: Reinforcement Learning and Bandits}

- Comparison-based Conversational Recommender System with Relative Bandit Feedback. 1400 Zhihui Xie (Shanghai Jiao Tong University), Tong Yu (Carnegie Mellon University), Canzhe Zhao (Shandong University), Shuai Li (Shanghai fiao Tong University)

- When and Whom to Collaborate with in a Changing Environment:

A Collaborative Dynamic Bandit Solution .

Chuanhao Li (University of Virginia), Qingyun Wu (University of Virginia),

Hongning Wang (University of Virginia)

- Glider: A Reinforcement Learning Approach to Extract UI Scripts from Websites

Yuanchun Li (Microsoft Research), Oriana Riva (Microsoft Research)

- Unified Conversational Recommendation Policy Learning via Graph-based

Reinforcement Learning

Yang Deng (The Chinese University of Hong Kong), Yaliang Li (Alibaba Group),

Fei Sun (Alibaba Group), Bolin Ding (Alibaba Group), Wai Lam (The Chinese University of Hong Kong)

\section{Session 6C: Natural Language and Semantics}

- Conversations Powered by Cross-Lingual Knowledge.

Weiwei Sun (Shandong University), Chuan Meng (Shandong University),

Qi Meng (Microsoft Research Asia), Zhaochun Ren (Shandong University),

Pengjie Ren (Shandong University), Zhumin Chen (Shandong University),

Maarten de Rijke (University of Amsterdam \& Ahold Delhaize Research)

- Transformer Reasoning Network for Personalized Review Summarization

Hongyan Xu (Tianjin University), Hongtao Liu (Tianjin University),

Pengfei Jiao (Tianjin University), Wenjun Wang (Tianjin University \& Shihezi University)

- Leveraging Lead Bias for Zero-shot Abstractive News Summarization

Chenguang Zhu (Microsoft), Ziyi Yang (Stanford University),

Robert Gmyr (Microsoft), Michael Zeng (Microsoft), Xuedong Huang (Microsoft)

- Retrieving Complex Tables with Multi-Granular Graph Representation Learning

Fei Wang (University of Southern California), Kexuan Sun (University of Southern California),

Muhao Chen (University of Southern California), Jay Pujara (University of Southern California),

Pedro Szekely (University of Southern California)

\section{Session 6D: IR Models}

- TILDE: Term Independent Likelihood moDEI for Passage Re-ranking 1483 Shengyao Zhuang (The University of Queensland), Guido Zuccon (The University of Queensland)

- Path-based Deep Network for Candidate Item Matching in Recommenders 1493 Houyi Li (Alibaba Group), Zhihong Chen (Alibaba Group), Chenliang Li (Wuhan University), Rong Xiao (Alibaba Group), Hongbo Deng (Alibaba Group), Peng Zhang (Alibaba Group), Yongchao Liu (Ant Group), Haihong Tang (Alibaba Group) 
- Optimizing Dense Retrieval Model Training with Hard Negatives

Jingtao Zhan (BNRist, DCST, Tsinghua University), Jiaxin Mao (GSAI, Renmin University of China),

Yiqun Liu (BNRist, DCST, Tsinghua University),

Jiafeng Guo (University of Chinese Academy of Sciences \& Institute of Computing Technology, CAS),

Min Zhang (BNRist, DCST, Tsinghua University), Shaoping Ma (BNRist, DCST, Tsinghua University)

- B-PROP: Bootstrapped Pre-training with Representative Words Prediction

for Ad-hoc Retrieval

Xinyu Ma (Institute of Computing Technology, Chinese Academy of Sciences \& University of Chinese Academy of Sciences),

Jiafeng Guo (Institute of Computing Technology, Chinese Academy of Sciences \& University of Chinese Academy of Sciences),

Ruqing Zhang (Institute of Computing Technology, Chinese Academy of Sciences \& University of Chinese

Academy of Sciences), Yixing Fan (Institute of Computing Technology, Chinese Academy of Sciences \& University

of Chinese Academy of Sciences),

Yingyan Li (Institute of Computing Technology, Chinese Academy of Sciences \& University of Chinese Academy of Sciences), Xueqi Cheng (Institute of Computing Technology, Chinese Academy of Sciences \& University of Chinese Academy of Sciences)

\section{Session 6E: Evaluation}

- Standing in Your Shoes: External Assessments for Personalized Recommender Systems.

Hongyu Lu (Tsinghua University), Weizhi Ma (Tsinghua University),

Min Zhang (Tsinghua University), Maarten de Rijke (University of Amsterdam \& Ahold Delhaize),

Yiqun Liu (Tsinghua University), Shaoping Ma (Tsinghua University)

- Evaluation Measures Based on Preference Graphs 1534

Charles L.A. Clarke (University of Waterloo), Chengxi Luo (University of Waterloo),

Mark D. Smucker (University of Waterloo)

- Do Affective Cues Validate Behavioural Metrics for Search?

Daniel McDuff (Microsoft), Paul Thomas (Microsoft), Nick Craswell (Microsoft),

Kael Rowan (Microsoft), Mary Czerwinski (Microsoft)

\section{Perspectives Papers}

- Current Challenges and Future Directions in Podcast Information Access. 1554

Rosie Jones (Spotify), Hamed Zamani (University of Massachusetts, Amherst),

Markus Schedl (fohannes Kepler University), Ching-Wei Chen (Spotify), Sravana Reddy (Spotify),

Ann Clifton (Spotify), Jussi Karlgren (Spotify), Helia Hashemi (University of Massachusetts, Amherst),

Aasish Pappu (Spotify), Zahra Nazari (Spotify), Longqi Yang (Microsoft),

Oguz Semerci (Spotify), Hugues Bouchard (Spotify), Ben Carterette (Spotify)

- MS MARCO: Benchmarking Ranking Models in the Large-Data Regime 1566

Nick Craswell (Microsoft), Bhaskar Mitra (Microsoft \& University College London),

Emine Yilmaz (University College London), Daniel Campos (University of Illinois at Urbana-Champaign),

Jimmy Lin (University of Waterloo \& Microsoft)

- Towards Multi-Modal Conversational Information Seeking

Yashar Deldjoo (Polytechnic University of Bari), Johanne R. Trippas (University of Melbourne),

Hamed Zamani (University of Massachusetts, Amherst)

\section{Short Research Papers I}

- AMM: Attentive Multi-field Matching for News Recommendation .

Qi Zhang (Noah's Ark Lab, Huawei), Qinglin Jia (Noah's Ark Lab, Huawei),

Chuyuan Wang (Noah's Ark Lab, Huawei), Jingjie Li (Noah's Ark Lab, Huawei),

Zhaowei Wang (Noah's Ark Lab, Huawei), Xiuqiang He (Noah's Ark Lab, Huawei)

- An ALBERT-based Similarity Measure for Mathematical Answer Retrieval 1593

Anja Reusch (Technische Universität Dresden), Maik Thiele (Technische Universität Dresden),

Wolfgang Lehner (Technische Universität Dresden)

- An Exploration of Tester-based Evaluation of User Simulators for Comparing Interactive Retrieval Systems.

Sahiti Labhishetty (University of Illinois Urbana-Champaign),

Chengxiang Zhai (University of Illinois Urbana-Champaign) 
- APRF-Net: Attentive Pseudo-Relevance Feedback Network for Query Categorization 1603 Ali Ahmadvand (Emory University), Sayyed M. Zahiri (The Home Depot), Simon Hughes (The Home Depot), Khalifeh Al Jadda (The Home Depot), Surya Kallumadi (The Home Depot), Eugene Agichtein (Emory University)

- Augmenting Sequential Recommendation with Pseudo-Prior Items via Reversely Pre-training Transformer 1608 Zhiwei Liu (University of Illinois at Chicago), Ziwei Fan (University of Illinois at Chicago), Yu Wang (University of Illinois at Chicago), Philip S. Yu (University of Illinois at Chicago)

- Cluster-Based Bandits: Fast Cold-Start for Recommender System New Users 1613 Sulthana Shams (Trinity College Dublin), Daron Anderson (Trinity College Dublin), Douglas Leith (Trinity College Dublin)

- Contextualized Offline Relevance Weighting for Efficient and Effective Neural Retrieval.. 1617 Xuanang Chen (University of Chinese Academy of Sciences), Ben He (University of Chinese Academy of Sciences), Kai Hui (Amazon Alexa), Yiran Wang (University of Chinese Academy of Sciences), Le Sun (Institute of Software, Chinese Academy of Sciences), Yingfei Sun (University of Chinese Academy of Sciences)

- Conversational vs Traditional: Comparing Search Behavior and Outcome in Legal Case Retrieval. 1622 Bulou Liu (Tsinghua University), Yueyue Wu (Tsinghua University), Yiqun Liu (Tsinghua University), Fan Zhang (Tsinghua University), Yunqiu Shao (Tsinghua University), Chenliang Li (Wuhan University), Min Zhang (Tsinghua University), Shaoping Ma (Tsinghua University)

- Counterfactual Explanations for Neural Recommenders. 1627 Khanh Hiep Tran (Max Planck Institute for Informatics), Azin Ghazimatin (Max Planck Institute for Informatics), Rishiraj Saha Roy (Max Planck Institute for Informatics)

- Cross-Batch Negative Sampling for Training Two-Tower Recommenders. 1632 Jinpeng Wang (Tsinghua University), Jieming Zhu (Huawei Noah's Ark Lab), Xiuqiang He (Huawei Noah's Ark Lab)

- De-Biased Modeling of Search Click Behavior with Reinforcement Learning 1637 Jianghong Zhou (Emory University), Sayyed M. Zahiri (The Home Depot), Simon Hughes (The Home Depot), Khalifeh Al Jadda (The Home Depot), Surya Kallumadi (The Home Depot), Eugene Agichtein (Emory University)

- DOZEN: Cross-Domain Zero Shot Named Entity Recognition with Knowledge Graph 1642 Hoang-Van Nguyen (PayPal), Francesco Gelli (PayPal), Soujanya Poria (Singapore University of Technology \& Design)

- Dual Unbiased Recommender Learning for Implicit Feedback 1647 Jae-woong Lee (Sungkyunkwan University), Seongmin Park (Sungkyunkwan University), Jongwuk Lee (Sungkyunkwan University)

- Empowering News Recommendation with Pre-trained Language Models 1652 Chuhan Wu (Tsinghua University), Fangzhao Wu (Microsoft Research Asia), Tao Qi (Tsinghua University), Yongfeng Huang (Tsinghua University)

- Entangled Bidirectional Encoder to Autoregressive Decoder for Sequential Recommendation .... 1657 Taegwan Kang (Seoul National University), Hwanhee Lee (Seoul National University), Byeongjin Choe (Seoul National University), Kyomin Jung (Seoul National University)

- Entity Retrieval Using Fine-Grained Entity Aspects 1662 Shubham Chatterjee (University of New Hampshire), Laura Dietz (University of New Hampshire)

- Evaluating the Predictivity of IR Experiments 1667 Lida Rashidi (The University of Melbourne), Justin Zobel (The University of Melbourne), Alistair Moffat (The University of Melbourne)

- FedCMR: Federated Cross-Modal Retrieval 1672 Linlin Zong (Dalian University of Technology), Qiujie Xie (Dalian University of Technology), Jiahui Zhou (Dalian University of Technology), Peiran Wu (Dalian University of Technology), Xianchao Zhang (Dalian University of Technology), Bo Xu (Dalian University of Technology) 
- Gazetteer Enhanced Named Entity Recognition for Code-Mixed Web Queries

Besnik Fetahu (Amazon.com, Inc.), Anjie Fang (Amazon.com, Inc.),

Oleg Rokhlenko (Amazon.com, Inc.), Shervin Malmasi (Amazon.com, Inc.)

- Hyperbolic Online Time Stream Modeling

Ramit Sawhney (Indraprastha Institute of Information Technology Delhi),

Shivam Agarwal (Manipal Institute of Technology), Megh Thakkar (BITS Pilani),

Arnav Wadhwa (Indraprastha Institute of Information Technology Delhi),

Rajiv Shah (Indraprastha Institute of Information Technology Delhi)

- ICAI-SR: Item Categorical Attribute Integrated Sequential Recommendation

Xu Yuan (Institute of Computing Technology, Chinese Academy of Sciences),

Dongsheng Duan (Institute of Computing Technology, Chinese Academy of Sciences),

Lingling Tong (National Computer Network Emergency Response Technical Team),

Lei Shi (National Computer Network Emergency Response Technical Team),

Cheng Zhang (Institute of Computing Technology, Chinese Academy of Sciences)

- Identifying Queries in Instant Search Logs

Markus Fischer (Friedrich-Schiller-Universität Jena), Kristof Komlossy (Bauhaus-Universität Weimar),

Benno Stein (Bauhaus-Universität Weimar), Martin Potthast (Leipzig University),

Matthias Hagen (Martin-Luther-Universität Halle-Wittenberg)

- Improving Transformer-Kernel Ranking Model Using Conformer and Query Term Independence

Bhaskar Mitra (Microsoft), Sebastian Hofstätter (TU Wien),

Hamed Zamani (University of Massachusetts, Amherst), Nick Craswell (Microsoft)

- Info-flow Enhanced GANs for Recommender

Yuan Lin (Dalian University of Technology), Zhang Xie (Dalian University of Technology),

Bo Xu (Dalian University of Technology), Kan Xu (Dalian University of Technology),

Hongfei Lin (Dalian University of Technology)

- Investigating Session Search Behavior with Knowledge Graphs.

Xiangsheng Li (Tsinghua University), Maarten de Rijke (University of Amsterdam \& Ahold Delhaize),

Yiqun Liu (Tsinghua University), Jiaxin Mao (Renmin University of China),

Weizhi Ma (Tsinghua University), Min Zhang (Tsinghua University), Shaoping Ma (Tsinghua University)

- Is Query Performance Prediction With Multiple Query Variations Harder

Than Topic Performance Prediction?

Oleg Zendel (RMIT University), J. Shane Culpepper (RMIT University),

Falk Scholer (RMIT University)

- Joint Learning of Deep Retrieval Model and Product Quantization based Embedding Index.

Han Zhang (FD.com), Hongwei Shen (FD.com Silicon Valley Research Center),

Yiming Qiu (JD.com), Yunjiang Jiang (FD.com Silicon Valley Research Center),

Songlin Wang (FD.com), Sulong Xu ( $(J D$.com), Yun Xiao (FD.com Silicon Valley Research Center),

Bo Long (FD.com), Wen-Yun Yang ( $(J D$.com Silicon Valley Research Center)

- Learning Passage Impacts for Inverted Indexes

Antonio Mallia (New York University), Omar Khattab (Stanford University),

Torsten Suel (New York University), Nicola Tonellotto (Università di Pisa)

- Learning Robust Dense Retrieval Models from Incomplete Relevance Labels

Prafull Prakash (University of Massachusetts, Amherst), Julian Killingback (University of Massachusetts, Amherst),

Hamed Zamani (University of Massachusetts, Amherst)

- Lighter and Better: Low-Rank Decomposed Self-Attention Networks for Next-Item

Recommendation

Xinyan Fan (Renmin University of China), Zheng Liu (Microsoft Research Asia),

Jianxun Lian (Microsoft Research Asia), Wayne Xin Zhao (Renmin University of China),

Xing Xie (Microsoft Research Asia), Ji-Rong Wen (Renmin University of China)

- Motif-aware Sequential Recommendation.

Zeyu Cui (Institute of Automation, Chinese Academy of Science \& University of Chinese Academy of Sciences),

Yinjiang Cai (University of Chinese Academy of Sciences \& Institute of Automation, Chinese Academy of Sciences),

Shu Wu (Institute of Automation, Chinese Academy of Science \& University of Chinese Academy of Sciences),

Xibo Ma (Institute of Automation, Chinese Academy of Science \& University of Chinese Academy of Sciences),

Liang Wang (Institute of Automation, Chinese Academy of Science \& University of Chinese Academy of Sciences), 
- Neural Representations in Hybrid Recommender Systems: Prediction versus Regularization.

Ramin Raziperchikolaei (Rakuten, Inc.), Tianyu Li (Rakuten, Inc.), Young-joo Chung (Rakuten, Inc.)

- On the Orthogonality of Bias and Utility in Ad hoc Retrieval.

Amin Bigdeli (Ryerson University), Negar Arabzadeh (University of Waterloo),

Shirin Seyedsalehi (Ryerson University), Morteza Zihayat (Ryerson University),

Ebrahim Bagheri (Ryerson University)

- Passage Retrieval for Outside-Knowledge Visual Question Answering

Chen Qu (University of Massachusetts, Amherst), Hamed Zamani (University of Massachusetts, Amherst),

Liu Yang (University of Massachusetts, Amherst), W. Bruce Croft (University of Massachusetts, Amherst),

Erik Learned-Miller (University of Massachusetts, Amherst)

- Predicting Links on Wikipedia with Anchor Text Information

Robin Brochier (Aix Marseille Université, Université de Toulon),

Frédéric Béchet (Aix Marseille Université, Université de Toulon)

- Propensity-Independent Bias Recovery in Offline Learning-to-Rank Systems

Zohreh Ovaisi (University of Illinois at Chicago), Kathryn Vasilaky (California Polytechnic State University),

Elena Zheleva (University of Illinois at Chicago)

- Revisiting the Tag Relevance Prediction Problem.

Denis Kotkov (University of Helsinki), Alexandr Maslov (Åbo Akademi University),

Mats Neovius (Åbo Akademi University)

- RMBERT: News Recommendation via Recurrent Reasoning Memory Network over BERT 1773 Qinglin Jia (Noah's Ark Lab, Huawei), Jingjie Li (Noah's Ark Lab, Huawei), Qi Zhang (Noah's Ark Lab, Huawei), Xiuqiang He (Noah's Ark Lab, Huawei), Jieming Zhu (Noah's Ark Lab, Huawei)

- Robust Neural Text Classification and Entailment via Mixup Regularized Adversarial Training

Jiahao Zhao (Institute of Automation, Chinese Academy of Sciences \& University of Chinese Academy of Sciences),

Penghui Wei (Institute of Automation, Chinese Academy of Sciences \& University of Chinese Academy of

Sciences),

Wenji Mao (Institute of Automation, Chinese Academy of Sciences \& University of Chinese Academy of Sciences)

- Sequential Recommendation for Cold-start Users with Meta Transitional Learning 1783

Jianling Wang (Texas A\&M University), Kaize Ding (Arizona State University),

James Caverlee (Texas A\&M University)

- Social Recommendation with Implicit Social Influence.

Changhao Song (College of Intelligence and Computing, Tianjin University \& People's Daily Online),

Bo Wang (College of Intelligence and Computing, Tianjin University \& People's Daily Online),

Qinxue Jiang (Newcastle University),

Yehua Zhang (College of Intelligence and Computing, Tianjin University),

Ruifang He (College of Intelligence and Computing, Tianjin University \& People's Daily Online),

Yuexian Hou (College of Intelligence and Computing, Tianjin University)

- Synthetic Target Domain Supervision for Open Retrieval QA

Revanth Gangi Reddy (University of Illinois at Urbana Champaign), Bhavani Iyer (IBM Research AI),

Md Arafat Sultan (IBM Research AI), Rong Zhang (IBM Research AI), Avirup Sil (IBM Research AI),

Vittorio Castelli (IBM Research AI), Radu Florian (IBM Research AI), Salim Roukos (IBM Research AI)

- Temporal Augmented Graph Neural Networks for Session-Based Recommendations. 1798

Huachi Zhou (The Hong Kong Polytechnic University), Qiaoyu Tan (The Hong Kong Polytechnic University),

Xiao Huang (The Hong Kong Polytechnic University), Kaixiong Zhou (Texas A\&M University),

Xiaoling Wang (East China Normal University)

- Text-to-Text Multi-view Learning for Passage Re-ranking

Jia-Huei Ju (Academia Sinica), Jheng-Hong Yang (University of Waterloo), Chuan-Ju Wang (Academia Sinica)

- The Winner Takes it All: Geographic Imbalance and Provider (Un)fairness

in Educational Recommender Systems

Elizabeth Gómez (Universitat de Barcelona), Carlos Shui Zhang (Universitat de Barcelona),

Ludovico Boratto (University of Cagliari), Maria Salamó (Universitat de Barcelona),

Mirko Marras (EPFL) 
- Transfer-Meta Framework for Cross-domain Recommendation to Cold-Start Users

Yongchun Zhu (Institute of Computing Technology, Chinese Academy of Sciences \& University of Chinese Academy of Sciences), Kaikai Ge (Tencent),

Fuzhen Zhuang (Beihang University \& Xiamen Data Intelligence Academy of ICT), Ruobing Xie (Tencent),

Dongbo Xi (Institute of Computing Technology, Chinese Academy of Sciences \& University of Chinese Academy of

Sciences),

Xu Zhang (Tencent), Leyu Lin (Tencent),

Qing He (Institute of Computing Technology, Chinese Academy of Sciences \& University of Chinese Academy of Sciences)

- Underestimation Refinement: A General Enhancement Strategy for Exploration in Recommendation Systems

Yuhai Song (fD.com), Lu Wang (FD.com), Haoming Dang (fD.com), Weiwei Zhou (FD.com),

Jing Guan (FD.com), Xiwei Zhao (FD.com), Changping Peng (fD.com), Yongjun Bao (FD.com),

Jingping Shao (FD.com)

- Web Document Encoding for Structure-Aware Keyphrase Extraction

Jihyuk Kim (Yonsei University), Young-In Song (Naver Corp), Seung-won Hwang (Seoul National University)

\section{Short Research Papers II}

- Adapted Graph Reasoning and Filtration for Description-Image Retrieval

Shiqian Chen (Alibaba Group), Zhiling Luo (Alibaba Group), Yingqi Gao (Alibaba Group),

Wei Zhou (Alibaba Group), Chenliang Li (School of Cyber Science and Engineering, Wuhan University),

Haiqing Chen (Alibaba Group)

- Affective Dependency Graph for Sarcasm Detection

Chenwei Lou (Harbin Institute of Technology),

Bin Liang (Joint lab of CMS-HITSZ, Harbin Institute of Technology, Shenzhen), Lin Gui (University of Warwick),

Yulan He (University of Warwick), Yixue Dang (China Merchants Securities Co., Ltd.),

Ruifeng Xu (Harbin Institute of Technology)

- Automatic Form Filling with Form-BERT

Gilad Fuchs (eBay Research), Haggai Roitman (eBay Research), Matan Mandelbrod (eBay Research)

- Circumstances enhanced Criminal Court View Generation 1855

Linan Yue (University of Science and Technology of China),

Qi Liu (University of Science and Technology of China), Han Wu (University of Science and Technology of China),

Yanqing An (University of Science and Technology of China),

Li Wang (University of Science and Technology of China),

Senchao Yuan (University of Science and Technology of China), Dayong Wu (IFLYTEK)

- Cross Interaction Network for Natural Language Guided Video Moment Retrieval 1860

Xinli Yu (Temple University), Mohsen Malmir (Amazon.com), Xin He (Amazon.com),

Jiangning Chen (Amazon.com), Tong Wang (Amazon.com), Yue Wu (Amazon.com),

Yue Liu (Amazon.com), Yang Liu (Amazon.com)

- Cross-Graph Attention Enhanced Multi-Modal Correlation Learning for Fine-Grained Image-Text Retrieval

Yi He (Huaqiao University \& Soochow University), Xin Liu (Huaqiao University),

Yiu-Ming Cheung (Hong Kong Baptist University), Shu-Juan Peng (Huaqiao University),

Jinhan Yi (Huaqiao University), Wentao Fan (Huaqiao University)

- DCSpell: A Detector-Corrector Framework for Chinese Spelling Error Correction

Jing Li (Sinovation Ventures), Gaosheng Wu (Sinovation Ventures), Dafei Yin (Sinovation Ventures), Haozhao Wang (HuaZhong University of Science and Technology), Yonggang Wang (Sinovation Ventures)

- Decoupling Representation and Regressor for Long-Tailed Information Cascade

Prediction

Fan Zhou (University of Electronic Science and Technology of China),

Liu Yu (University of Electronic Science and Technology of China),

Xovee Xu (University of Electronic Science and Technology of China), Goce Trajcevski (Iowa State University)

- Deep Music Retrieval for Fine-Grained Videos by Exploiting

Cross-Modal-Encoded Voice-Overs. 1880

Tingtian Li (Tencent), Zixun Sun (Tencent), Haoruo Zhang (Tencent), Jin Li (Tencent),

Ziming Wu (Tencent), Hui Zhan (Tencent), Yipeng Yu (Tencent),

Hengcan Shi (University of Electronic Science and Technology of China) 
- Deep Position-wise Interaction Network for CTR Prediction

Jianqiang Huang (Meituan), Ke Hu (Meituan), Qingtao Tang (Meituan),

Mingjian Chen (Meituan), Yi Qi (Meituan), Jia Cheng (Meituan), Jun Lei (Meituan)

- Deep User Match Network for Click-Through Rate Prediction

Zai Huang (Alibaba Group), Mingyuan Tao (Alibaba Group), Bufeng Zhang (Alibaba Group)

- Distant Supervision based Machine Reading Comprehension for Extractive

Summarization in Customer Service

Bing Ma (Beijing University of Posts and Telecommunications \& Meituan), Cao Liu (Meituan),

Jingyu Wang (Beijing University of Posts and Telecommunications), Shujie Hu (Meituan),

Fan Yang (Meituan), Xunliang Cai (Meituan), Guanglu Wan (Meituan), Jiansong Chen (Meituan),

Jianxin Liao (Beijing University of Posts and Telecommunications)

- Does BERT Pay Attention to Cyberbullying?

Fatma Elsafoury (University of the West of Scotland), Stamos Katsigiannis (Durham University),

Steven R. Wilson (University of Edinburgh), Naeem Ramzan (University of the West of Scotland)

- ECG Data Modeling and Analyzing via Deep Representation Learning and Nonparametric Hidden Markov Models

Jiaojiao Zhu (Huaqiao University \& Soochow University), Wentao Fan (Huaqiao University)

- Faster Index Reordering with Bipartite Graph Partitioning

Joel Mackenzie (The University of Melbourne), Matthias Petri (Amazon Alexa),

Alistair Moffat (The University of Melbourne)

- Follow the Prophet: Accurate Online Conversion Rate Prediction in the Face

of Delayed Feedback

Haoming Li (Chinese Academy of Sciences \& University of Chinese Academy of Sciences),

Feiyang Pan (Chinese Academy of Sciences \& University of Chinese Academy of Sciences),

Xiang Ao (Chinese Academy of Sciences \& University of Chinese Academy of Sciences),

Zhao Yang (Chinese Academy of Sciences \& University of Chinese Academy of Sciences),

Min Lu (Tencent), Junwei Pan (Tencent), Dapeng Liu (Tencent), Lei Xiao (Tencent),

Qing He (Institute of Computing Technology, Chinese Academy of Sciences \& University of Chinese Academy of Sciences)

- GAIPS: Accelerating Maximum Inner Product Search with GPU

Long Xiang (Southern University of Science and Technology \& Peng Cheng Laboratory),

Xiao Yan (Department of Computer Science and Engineering, Southern University of Science and Technology),

Lan Lu (Department of Computer Science and Engineering, Southern University of Science and Technology),

Bo Tang (Southern University of Science and Technology \& Peng Cheng Laboratory)

- Generalized Zero-shot Intent Detection via Commonsense Knowledge 1925

A.B. Siddique (University of California, Riverside), Fuad Jamour (University of California, Riverside),

Luxun Xu (University of California, Riverside), Vagelis Hristidis (University of California, Riverside)

- Graph-Structured Context Understanding for Knowledge-grounded Response Generation

Yanran Li (The Hong Kong Polytechnic University), Wenjie Li (The Hong Kong Polytechnic University),

Zhitao Wang (The Hong Kong Polytechnic University)

- Hierarchical Dependence-aware Evaluation Measures for Conversational Search 1935

Guglielmo Faggioli (University of Padova), Marco Ferrante (University of Padova),

Nicola Ferro (University of Padova), Raffaele Perego (National Research Council),

Nicola Tonellotto (University of Pisa)

- Improving Response Quality with Backward Reasoning in Open-domain Dialogue Systems.

Ziming Li (University of Amsterdam), Julia Kiseleva (Microsoft),

Maarten de Rijke (University of Amsterdam \& Ahold Delhaize)

- Knowledge Based Hyperbolic Propagation

Chang-You Tai (Academia Sinica), Chien-Kun Huang (Academia Sinica),

Liang-Ying Huang (Academia Sinica), Lun-Wei Ku (Academia Sinica) 
- Learning to Select Instance: Simultaneous Transfer Learning and Clustering

Zhaoxin Huan (Ant Group), Yulong Wang (Ant Group), Yong He (Ant Group), Xiaolu Zhang (Ant Group),

Chilin Fu (Ant Group), Weichang Wu (Ant Group), Jun Zhou (Ant Group), Ke Ding (Ant Group),

Liang Zhang (Ant Group), Linjian Mo (Ant Group)

- LPF: A Language-Prior Feedback Objective Function for De-biased Visual

Question Answering .

Zujie Liang (Sun Yat-sen University), Haifeng Hu (Sun Yat-sen University), Jiaying Zhu (Sun Yat-sen University)

- LS-DST: Long and Sparse Dialogue State Tracking with Smart History Collector in Insurance Marketing 1960

Liqiang Song (Ping An Technology Co, LTd), Mengqiu Yao (Ping An Technology Co, LTd),

Ye Bi (Ping An Technology Co, LTd), Zhenyu Wu (Ping An Technology Co, LTd),

Jianming Wang (Ping An Technology Co, LTd), Jing Xiao (Ping An Technology Co, LTd),

Juan Wen (Ping An Life Insurance Company), Xin Yu (Ping An Life Insurance Company)

- Medical Triage Chatbot Diagnosis Improvement via Multi-relational Hyperbolic Graph Neural Network

Zheng Liu (University of Illinois at Chicago), Xiaohan Li (University of Illinois at Chicago),

Zeyu You (Tencent), Tao Yang (Tencent), Wei Fan (Tencent), Philip Yu (University of Illinois at Chicago)

- Meta-Learned Specific Scenario Interest Network for User Preference Prediction 1970

Yinan Sun (Beijing University of Posts and Telecommunications),

Kang Yin (University of Chinese Academy of Sciences), Hehuan Liu (Beijing University of Technology),

$\mathrm{Si} \mathrm{Li} \mathrm{(Beijing} \mathrm{University} \mathrm{of} \mathrm{Posts} \mathrm{and} \mathrm{Telecommunications),}$

Yajing Xu (Beijing University of Posts and Telecommunications),

Jun Guo (Beijing University of Posts and Telecommunications)

- On the Privacy of Federated Pipelines

Reza Nasirigerdeh (Technical University of Munich),

Reihaneh Torkzadehmahani (Technical University of Munich),

Jan Baumbach (University of Hamburg \& University of Southern Denmark),

David B. Blumenthal (Technical University of Munich)

- On the Two-Sample Randomisation Test for IR Evaluation

Tetsuya Sakai (Waseda University)

- Position Enhanced Mention Graph Attention Network for Dialogue Relation Extraction...

Xinwei Long (Institute of Software Chinese Academy of Sciences \& University of Chinese Academy of Sciences),

Shuzi Niu (Institute of Software Chinese Academy of Sciences),

Yucheng Li (Institute of Software Chinese Academy of Sciences)

- Predicting Patient Readmission Risk from Medical Text via Knowledge

Graph Enhanced Multiview Graph Convolution

Qiuhao Lu (University of Oregon), Thien Huu Nguyen (University of Oregon),

Dejing Dou (University of Oregon \& Baidu Research)

- Predicting User Demography and Device from News Comments

Ohad Rozen (Bar Ilan University), Joel Oren (Bosch Center for AI), Ariel Raviv (Yahoo Research)

- Proactive Retrieval-based Chatbots based on Relevant Knowledge and Goals

Yutao Zhu (Université de Montréal), Jian-Yun Nie (Université de Montréal),

Kun Zhou (Renmin University of China), Pan Du (Université de Montréal),

Hao Jiang (Huawei Poisson Lab.), Zhicheng Dou (Renmin University of China)

- Pseudo Siamese Network for Few-shot Intent Generation

Congying Xia (University of Illinois at Chicago), Caiming Xiong (Salesforce Research),

Philip Yu (University of Illinois at Chicago)

- Retrieving Implicit Information for Stock Movement Prediction

Tsun-Hsien Tang (National Taiwan University), Chung-Chi Chen (National Taiwan University),

Hen-Hsen Huang (National Chengchi University),

Hsin-Hsi Chen (National Taiwan University \& MOST Joint Research Center for AI Technology and All Vista

Healthcare)

- Retrieving Skill-Based Teams from Collaboration Networks

Radin Hamidi Rad (Ryerson University), Ebrahim Bagheri (Ryerson University),

Mehdi Kargar (Ryerson University), Divesh Srivastava (AT\&T Labs-Research),

Jaroslaw Szlichta (Ontario Tech University) 
- Rumor Detection on Social Media with Event Augmentations

Zhenyu He (University of Electronic Science and Technology of China),

Ce Li (University of Electronic Science and Technology of China),

Fan Zhou (University of Electronic Science and Technology of China),

Yi Yang (Hong Kong University of Science and Technology)

- Similar Trademark Detection via Semantic, Phonetic and Visual Similarity Information ..... 2025 Yingchi Liu (Alibaba Group), Quanzhi Li (Alibaba Group), Changlong Sun (Alibaba Group), Luo Si (Alibaba Group)

- Structured Fine-Tuning of Contextual Embeddings for Effective Biomedical Retrieval 2031 Alberto Ueda (Federal University of Minas Gerais), Rodrygo L. T. Santos (Federal University of Minas Gerais), Craig Macdonald (University of Glasgow), Iadh Ounis (University of Glasgow)

- Towards a Better Tradeoff between Effectiveness and Efficiency in Pre-Ranking: A Learnable Feature Selection based Approach

Xu Ma (Alibaba Group), Pengjie Wang (Alibaba Group), Hui Zhao (Alibaba Group), Shaoguo Liu (Alibaba Group), Chuhan Zhao (Alibaba Group), Wei Lin (Alibaba Group), Kuang-Chih Lee (Alibaba Group), Jian Xu (Alibaba Group), Bo Zheng (Alibaba Group)

- Towards an Online Empathetic Chatbot with Emotion Causes 2041 Yanran Li (Xiaomi AI Lab), Ke Li (Xiaomi AI Lab), Hongke Ning (Xiaomi AI Lab), Xiaoqiang Xia (Xiaomi AI Lab), Yalong Guo (Xiaomi AI Lab), Chen Wei (Xiaomi AI Lab), Jianwei Cui (Xiaomi AI Lab), Bin Wang (Xiaomi AI Lab)

- User Feedback and Ranking in-a-Loop: Towards Self-Adaptive Dialogue Systems 2046 Chen Shi (Alibaba Group), Yuxiang Hu (Alibaba Group), Zengming Zhang (Alibaba Group), Liang Shao (Alibaba Group), Feijun Jiang (Alibaba Group)

- User Preference-aware Fake News Detection 2051 Yingtong Dou (University of Illinois at Chicago), Kai Shu (Illinois Institute of Technology), Congying Xia (University of Illinois at Chicago), Philip S. Yu (University of Illinois at Chicago), Lichao Sun (Lehigh University)

- Utility of Missing Concepts in Query-biased Summarization 2056 Sheikh Muhammad Sarwar (University of Massachusetts, Amherst), Felipe Moraes (Delft University of Technology), Jiepu Jiang (University of Wisconsin-Madison), James Allan (University of Massachusetts, Amherst)

- Variational Autoencoders for Top-K Recommendation with Implicit Feedback 2061 Bahare Askari (Ontario Tech University), Jaroslaw Szlichta (Ontario Tech University), Amirali Salehi-Abari (Ontario Tech University)

- Vera: Prediction Techniques for Reducing Harmful Misinformation in Consumer Health Search . 2066 Ronak Pradeep (University of Waterloo), Xueguang Ma (University of Waterloo), Rodrigo Nogueira (University of Waterloo), Jimmy Lin (University of Waterloo)

- Visual Question Rewriting for Increasing Response Rate 2071 Jiayi Wei (University Of California, Santa Cruz), Xilian Li (University Of California, Santa Cruz), Yi Zhang (University Of California, Santa Cruz), Xin Eric Wang (University Of California, Santa Cruz)

- X-2ch: Quad-Channel Collaborative Graph Network over Knowledge-Embedded Edges..... 2076 Kachun Lo (Tohoku Universiy), Tsukasa Ishigaki (Tohoku Universiy)

\section{Short Research Papers III}

- A Systematic Evaluation of Transfer Learning and Pseudo-labeling with BERT-based Ranking Models. 2081 Iurii Mokrii (HSE University), Leonid Boytsov (Bosch Center for Artificial Intelligence), Pavel Braslavski (Ural Federal University \& HSE University) 
- Abstractive Text Summarization with Hierarchical Multi-scale Abstraction

Modeling and Dynamic Memory.

Lihan Wang (Shenzhen Institutes of Advanced Technology, Chinese Academy of Sciences \& University of Chinese Academy of Sciences),

Min Yang (Shenzhen Institutes of Advanced Technology, Chinese Academy of Sciences),

Chengming Li (Shenzhen Institutes of Advanced Technology, Chinese Academy of Sciences),

Ying Shen (Sun Yat-Sen University), Ruifeng Xu (Harbin Institute of Technology)

- Accelerating Neural Architecture Search for Natural Language Processing with Knowledge Distillation and Earth Mover's Distance. 2091

Jianquan Li (Yibin University), Xiaokang Liu (Beijing Ultrapower Software Co., Ltd.),

Sheng Zhang (National University of Defense Technology), Min Yang (SIAT, Chinese Academy of Sciences),

Ruifeng Xu (Harbin Institute of Technology), Fengqing Qin (Yibin University)

- Automated Graph Learning via Population Based Self-Tuning GCN

Ronghang Zhu (University of Georgia), Zhiqiang Tao (Santa Clara University),

Yaliang Li (Alibaba Group), Sheng Li (University of Georgia)

- AutoName: A Corpus-Based Set Naming Framework

Zhiqi Huang (University of Massachusetts, Amherst), Razieh Rahimi (University of Massachusetts, Amherst),

Puxuan Yu (University of Massachusetts, Amherst), Jingbo Shang (University of California, San Diego),

James Allan (University of Massachusetts, Amherst)

- Backretrieval: An Image-Pivoted Evaluation Metric for Cross-Lingual Text

Representations Without Parallel Corpora 2106

Mikhail Fain (Cookpad Ltd.), Niall Twomey (Cookpad Ltd.), Danushka Bollegala (The University of Liverpool)

- Bayesian Critiquing with Keyphrase Activation Vectors for VAE-based

Recommender Systems.

Hojin Yang (University of Toronto), Tianshu Shen (University of Toronto),

Scott Sanner (University of Toronto)

- Cheap and Good? Simple and Effective Data Augmentation for Low Resource

Machine Reading

Hoang Van (University of Arizona), Vikas Yadav (IBM Research),

Mihai Surdeanu (University of Arizona)

- CIFDM: Continual and Interactive Feature Distillation for Multi-Label Stream Learning … 2121 Yigong Wang (The University of Texas at Dallas), Zhuoyi Wang (The University of Texas at Dallas),

Yu Lin (The University of Texas at Dallas), Latifur Khan (The University of Texas at Dallas),

Dingcheng Li (Amazon Alexa AI)

- Clustering-Based Online News Topic Detection and Tracking Through Hierarchical Bayesian Nonparametric Models

Wentao Fan (Huaqiao University), Zhiyan Guo (Huaqiao University),

Nizar Bouguila (Concordia University), Wenjuan Hou (Huaqiao University)

- Communication Efficient Distributed Hypergraph Clustering

Chun Jiang Zhu (University of North Carolina Greensboro),

Qinqing Liu (University of Connecticut), Jinbo Bi (University of Connecticut)

- Composite Code Sparse Autoencoders for First Stage Retrieval

Carlos Lassance (Naver Labs Europe), Thibault Formal (Naver Labs Europe),

Stéphane Clinchant (Naver Labs Europe)

- ConsisRec: Enhancing GNN for Social Recommendation via Consistent Neighbor

Aggregation

Liangwei Yang (University of Illinois at Chicago), Zhiwei Liu (University of Illinois at Chicago),

Yingtong Dou (University of Illinois at Chicago), Jing Ma (Sichuan University),

Philip S. Yu (University of Illinois at Chicago)

- DSGPT: Domain-Specific Generative Pre-Training of Transformers for Text

Generation in E-commerce Title and Review Summarization.

Xueying Zhang (JD.com Silicon Valley Research Center), Yunjiang Jiang (JD.com Silicon Valley Research Center),

Yue Shang (FD.com Silicon Valley Research Center), Zhaomeng Cheng (JD.com Silicon Valley Research Center),

Chi Zhang (FD.com Silicon Valley Research Center), Xiaochuan Fan (FD.com Silicon Valley Research Center),

Yun Xiao (JD.com Silicon Valley Research Center), Bo Long (FD.com Silicon Valley Research Center) 
- Dual-View Distilled BERT for Sentence Embedding

Xingyi Cheng (Ant Group)

- Enhanced Representation Learning for Examination Papers with Hierarchical

Document Structure

Yixiao Ma (University of Science and Technology of China),

Shiwei Tong (University of Science and Technology of China),

Ye Liu (University of Science and Technology of China),

Likang $\mathrm{Wu}$ (University of Science and Technology of China),

Qi Liu (University of Science and Technology of China),

Enhong Chen (University of Science and Technology of China),

Wei Tong (University of Science and Technology of China),

Zi Yan (The National Education Examinations Authority of the People's Republic of China)

- Explicit Semantic Cross Feature Learning via Pre-trained Graph Neural Networks for CTR Prediction.

Feng Li (Alibaba Group), Bencheng Yan (Alibaba Group), Qingqing Long (Alibaba Group),

Pengjie Wang (Alibaba Group), Wei Lin (Alibaba Group), Jian Xu (Alibaba Group),

Bo Zheng (Alibaba Group),

- GemNN: Gating-enhanced Multi-Task Neural Networks with Feature Interaction

Learning for CTR Prediction

Hongliang Fei (Baidu Research), Jingyuan Zhang (Baidu Research), Xingxuan Zhou (Baidu Inc.),

Junhao Zhao (Baidu Inc.), Xinyang Qi (Baidu Inc.), Ping Li (Baidu Research)

- Graph Learning Regularization and Transfer Learning for Few-Shot Event Detection

Viet Dac Lai (University of Oregon), Minh Van Nguyen (University of Oregon),

Thien Huu Nguyen (University of Oregon), Franck Dernoncourt (Adobe Inc.)

- Graph Pooling via Coarsened Graph Infomax

Yunsheng Pang (The University of Melbourne),

Yunxiang Zhao (National University of Defense Technology \& The University of Melbourne),

Dongsheng Li (National University of Defense Technology)

- GraphPAS: Parallel Architecture Search for Graph Neural Networks.

Jiamin Chen (Central South University), Jianliang Gao (Central South University),

Yibo Chen (State Grid Hunan ElectricPower Company Limited),

Moctard Babatounde Oloulade (Central South University), Tengfei Lyu (Central South University),

Zhao Li (Alibaba Group)

- Hierarchically Modeling Micro and Macro Behaviors via Multi-Task Learning for Conversion Rate Prediction.

Hong Wen (Alibaba Group), Jing Zhang (The University of Sydney), Fuyu Lv (Alibaba Group),

Wentian Bao (Alibaba Group), Tianyi Wang (Alibaba Group), Zulong Chen (Alibaba Group)

- Improving Bi-encoder Document Ranking Models with Two Rankers and Multi-teacher Distillation

Jaekeol Choi (Seoul National University \& Naver Corp.), Euna Jung (Seoul National University),

Jangwon Suh (Seoul National University), Wonjong Rhee (Seoul National University)

- Improving Neural Text Style Transfer by Introducing Loss Function Sequentiality

Chinmay Rane (Normandie University \& ABV-Indian Institute of Information Technology and Management

Gwalior), Gaël Dias (Normandie Univ, UNICAEN, ENSICAEN, CNRS, GREYC),

Alexis Lechervy (Normandie Univ, UNICAEN, ENSICAEN, CNRS, GREYC),

Asif Ekbal (Indian Institute of Technology Patna)

- Inductive Representation Learning in Temporal Networks via Mining

Neighborhood and Community Influences

Meng Liu (Heilongjiang University), Yong Liu (Heilongjiang University)

- KeyBLD: Selecting Key Blocks with Local Pre-ranking for Long Document Information Retrieval...

Minghan Li (University Grenoble Alpes, CNRS, LIG), Eric Gaussier (University Grenoble Alpes, CNRS, LIG)

- Knowledge Graph Embedding via Metagraph Learning

Chanyoung Chung (Korea Advanced Institute of Science and Technology),

Joyce Jiyoung Whang (Korea Advanced Institute of Science and Technology) 
- Learning Early Exit Strategies for Additive Ranking Ensembles

Francesco Busolin (Ca' Foscari University of Venice), Claudio Lucchese (Ca' Foscari University of Venice),

Franco Maria Nardini (ISTI-CNR), Salvatore Orlando (Ca' Foscari University of Venice),

Raffaele Perego (ISTI-CNR), Salvatore Trani (ISTI-CNR)

- LSTPR: Graph-based Matrix Factorization with Long Short-term Preference Ranking 2222 Chih-Hen Lee (Academia Sinica), Jun-En Ding (Academia Sinica),

Chih-Ming Chen (National Chengchi University), Jing-Kai Lou (KKStream Limited),

Ming-Feng Tsai (National Chengchi University), Chuan-Ju Wang (Academia Sinica)

- Maximal Multipolarized Cliques Search in Signed Networks

Jie Gao (Shaanxi Normal University), Fei Hao (Shaanxi Normal University \& University of Exeter),

Geyong Min (University of Exeter), Zhipeng Cai (Georgia State University)

- MetaP: Meta Pattern Learning for One-Shot Knowledge Graph Completion

Zhiyi Jiang (Central South University), Jianliang Gao (Central South University),

Xinqi Lv (Central South University)

- MSSM: A Multiple-level Sparse Sharing Model for Efficient Multi-Task Learning

Ke Ding (Ant Group), Xin Dong (Ant Group), Yong He (Ant Group), Lei Cheng (Ant Group),

Chilin Fu (Ant Group), Zhaoxin Huan (Ant Group), Hai Li (Ant Group), Tan Yan (Ant Group),

Liang Zhang (Ant Group), Xiaolu Zhang (Ant Group), Linjian Mo (Ant Group)

- NIP-GCN: An Augmented Graph Convolutional Network with Node Interaction Patterns . 2242 Manish Chandra (Indian Institute of Technology Kharagpur), Debasis Ganguly (University of Glasgow),

Pabitra Mitra (Indian Institute of Technology Kharagpur),

Bithika Pal (Indian Institute of Technology Kharagpur), James Thomas (University College London)

- Podcast Metadata and Content: Episode Relevance and Attractiveness in Ad Hoc Search .... 2247 Ben Carterette (Spotify), Rosie Jones (Spotify), Gareth F. Jones (Dublin City University),

Maria Eskevich (CLARIN), Sravana Reddy (Spotify), Ann Clifton (Spotify), Yongze Yu (Spotify),

Jussi Karlgren (Spotify), Ian Soboroff (NIST)

- Propensity-scored Probabilistic Label Trees

Marek Wydmuch (Poznan University of Technology),

Kalina Jasinska-Kobus (ML Research at Allegro.pl \& Poznan University of Technology),

Rohit Babbar (Aalto University), Krzysztof Dembczynski (Yahoo! Research \& Poznan University of Technology)

- ReadsRE: Retrieval-Augmented Distantly Supervised Relation Extraction. 2257

Yue Zhang (Baidu Research), Hongliang Fei (Baidu Research), Ping Li (Baidu Research)

- Regularized Dual-PPMI Co-clustering for Text Data. Séverine Affeldt (Université de Paris, CNRS, Centre Borelli), Lazhar Labiod (Université de Paris, CNRS, Centre Borelli), Mohamed Nadif (Université de Paris, CNRS, Centre Borelli)

- RLNF: Reinforcement Learning based Noise Filtering for Click-Through Rate Prediction... 2268 Pu Zhao (Microsoft), Chuan Luo (Microsoft), Cheng Zhou (Microsoft), Bo Qiao (Microsoft), Jiale He (Microsoft), Liangjie Zhang (Microsoft), Qingwei Lin (Microsoft)

- SDG: A Simplified and Dynamic Graph Neural Network... Dongqi Fu (University of Illinois at Urbana-Champaign), Jingrui He (University of Illinois at Urbana-Champaign)

- Semantic Query Labeling Through Synthetic Query Generation 2278 Elias Bassani (Consorzio per il Trasferimento Tecnologico - C2T \& University of Milano-Bicocca), Gabriella Pasi (University of Milano-Bicocca)

- Significant Improvements over the State of the Art? A Case Study of the MS MARCO Document Ranking Leaderboard

Jimmy Lin (University of Waterloo and Microsoft AI \& Research),

Daniel Campos (University of Illinois at Urbana-Champaign), Nick Craswell (Microsoft AI \& Research),

Bhaskar Mitra (Microsoft AI \& Research and University College London),

Emine Yilmaz (University College London)

- SPLADE: Sparse Lexical and Expansion Model for First Stage Ranking

Thibault Formal (Naver Labs Europe \& Sorbonne Université, LIP6),

Benjamin Piwowarski (Sorbonne Université, CNRS, LIP6), Stéphane Clinchant (Naver Labs Europe)

- Stopping Criteria for Technology Assisted Reviews based on Counting Processes

Alison Sneyd (University of Sheffield), Mark Stevenson (University of Sheffield) 
- Targeted Attack and Defense for Deep Hashing.

Xunguang Wang (Harbin Institute of Technology),

Zheng Zhang (Harbin Institute of Technology \& Peng Cheng Laboratory),

Guangming Lu (Harbin Institute of Technology),

Yong Xu (Harbin Institute of Technology \& Peng Cheng Laboratory)

- Understanding the Role of Affect Dimensions in Detecting Emotions from Tweets:

A Multi-task Approach

Rajdeep Mukherjee (IIT Kharagpur), Atharva Naik (IIT Kharagpur),

Sriyash Poddar (IIT Kharagpur), Soham Dasgupta (MAIS Bangalore), Niloy Ganguly (IIT Kharagpur)

- Unsupervised Ensemble Learning with Noisy Label Correction.....

Xupeng Zou (Xiamen University), Zhongnan Zhang (Xiamen University),

Zhen He (Beijing Institute of Basic Medical Sciences), Liang Shi (Xiamen University)

- Unsupervised Extractive Text Summarization with Distance-Augmented

Sentence Graphs

Jingzhou Liu (Carnegie Mellon University), Dominic J. D. Hughes (University of California, Berkeley),

Yiming Yang (Carnegie Mellon University)

- When Choice Happens: A Systematic Examination of Mouse Movement Length for Decision Making in Web Search

Lukas Brückner (Independent researcher), Ioannis Arapakis (Telefonica Research),

Luis A. Leiva (University of Luxembourg)

- Window Navigation with Adaptive Probing for Executing BlockMax WAND

Jinjin Shao (University of California, Santa Barbara), Yifan Qiao (University of California, Santa Barbara),

Shiyu Ji (University of California, Santa Barbara), Tao Yang (University of California, Santa Barbara)

\section{Resource Papers I}

- A Multilingual Dataset for Named Entity Recognition, Entity Linking and Stance Detection in Historical Newspapers.

Ahmed Hamdi (University of La Rochelle), Elvys Linhares Pontes (University of La Rochelle),

Emanuela Boros (University of La Rochelle), Thi Tuyet Hai Nguyen (University of La Rochelle),

Günter Hackl (Innsbruck University Innovations GmbH), Jose G. Moreno (University of Toulouse),

Antoine Doucet (University of La Rochelle)

- How Deep is your Learning: the DL-HARD Annotated Deep Learning Dataset 2335

Iain Mackie (University of Glasgow), Jeffrey Dalton (University of Glasgow),

Andrew Yates (Max Planck Institute for Informatics)

- LeCaRD: A Legal Case Retrieval Dataset for Chinese Law System

Yixiao Ma (Tsinghua University), Yunqiu Shao (Tsinghua University), Yueyue Wu (Tsinghua University),

Yiqun Liu (Tsinghua University), Ruizhe Zhang (Tsinghua University),

Min Zhang (Tsinghua University), Shaoping Ma (Tsinghua University)

- Morphologically Annotated Amharic Text Corpora

Tilahun Yeshambel (Addis Ababa University), Josiane Mothe (University de Toulouse),

Yaregal Assabie (Addis Ababa University)

- Pyserini: A Python Toolkit for Reproducible Information Retrieval Research with Sparse and Dense Representations.

Jimmy Lin (University of Waterloo), Xueguang Ma (University of Waterloo),

Sheng-Chieh Lin (University of Waterloo), Jheng-Hong Yang (University of Waterloo),

Ronak Pradeep (University of Waterloo), Rodrigo Nogueira (University of Waterloo)

- REGIS: A Test Collection for Geoscientific Documents in Portuguese .

Lucas Lima de Oliveira (UFRGS), Regis Kruel Romeu (CENPES/Petrobras), Viviane Pereira Moreira (UFRGS)

- TREC Deep Learning Track: Reusable Test Collections in the Large Data Regime 2369

Nick Craswell (Microsoft), Bhaskar Mitra (Microsoft), Emine Yilmaz (University College London),

Daniel Campos (University of Illinois, Urbana-Champaign),

Ellen M. Voorhees (National Institute of Standards and Technology),

Ian Soboroff (National Institute of Standards and Technology)

- WWW3E8: 259,000 Relevance Labels for Studying the Effect of Document Presentation Order for Relevance Assessors. 2376

Tetsuya Sakai (Waseda University), Sijie Tao (Waseda University), Zhaohao Zeng (Waseda University) 


\section{Resource Papers II}

- Advancements in the Music Information Retrieval Framework AMUSE over the Last Decade 2383

Igor Vatolkin (TU Dortmund University), Philipp Ginsel (TU Dortmund University), Günter Rudolph (TU Dortmund University)

- Conversational Entity Linking: Problem Definition and Datasets 2390 Hideaki Joko (Radboud University), Faegheh Hasibi (Radboud University), Krisztian Balog (University of Stavanger), Arjen P. de Vries (Radboud University)

- CopyCat: Near-Duplicates Within and Between the ClueWeb and the Common Crawl . 2398 Maik Fröbe (Martin-Luther-Universität Halle-Wittenberg), Janek Bevendorff (Bauhaus-Universität Weimar), Lukas Gienapp (Leipzig University), Michael Völske (Bauhaus-Universität Weimar), Benno Stein (Bauhaus-Universität Weimar), Martin Potthast (Leipzig University), Matthias Hagen (Martin-Luther-Universität Halle-Wittenberg)

- Elliot: A Comprehensive and Rigorous Framework for Reproducible Recommender Systems Evaluation.

Vito Walter Anelli (Politecnico di Bari), Alejandro Bellogin (Autónoma Madrid), Antonio Ferrara (Politecnico di Bari), Daniele Malitesta (Politecnico di Bari), Felice Antonio Merra (Politecnico di Bari), Claudio Pomo (Politecnico di Bari), Francesco Maria Donini (Università della Tuscia), Tommaso Di Noia (Politecnico di Bari)

- HOOPS: Human-in-the-Loop Graph Reasoning for Conversational Recommendation 2415 Zuohui Fu (Rutgers University), Yikun Xian (Rutgers University), Yaxin Zhu (Rutgers University), Shuyuan Xu (Rutgers University), Zelong Li (Rutgers University), Gerard de Melo (HPI/University of Potsdam), Yongfeng Zhang (Rutgers University)

- On the Quality of the TREC-COVID IR Test Collections 2422 Ellen M. Voorhees (National Institute of Standards and Technology), Kirk Roberts (The University of Texas Health Science Center at Houston)

- Simplified Data Wrangling with ir_datasets

Sean MacAvaney (University of Glasgow \& Georgetown University),

Andrew Yates (Max Planck Institute for Informatics), Sergey Feldman (Allen Institute for AI),

Doug Downey (Allen Institute for AI), Arman Cohan (Allen Institute for AI),

Nazli Goharian (Georgetown University)

- Wiki-Reliability: A Large Scale Dataset for Content Reliability on Wikipedia 2437 KayYen Wong (Outreachy), Miriam Redi (Wikimedia Foundation), Diego Saez-Trumper (Wikimedia Foundation)

- WIT: Wikipedia-based Image Text Dataset for Multimodal Multilingual Machine Learning 2443 Krishna Srinivasan (Google), Karthik Raman (Google), Jiecao Chen (Google), Michael Bendersky (Google), Marc Najork (Google)

\section{Resource Papers III}

- A Test Collection for Ad-hoc Dataset Retrieval. 2450 Makoto P. Kato (University of Tsukuba \& JST, PRESTO), Hiroaki Ohshima (University of Hyogo), Ying-Hsang Liu (Oslo Metropolitan University), Hsin-Liang Chen (Missouri University of Science and Technology)

- Booking.com Multi-Destination Trips Dataset 2457 Dmitri Goldenberg (Booking.com), Pavel Levin (Booking.com)

- EXTRA: Explanation Ranking Datasets for Explainable Recommendation 2463 Lei Li (Hong Kong Baptist University), Yongfeng Zhang (Rutgers University), Li Chen (Hong Kong Baptist University)

- Pchatbot: A Large-Scale Dataset for Personalized Chatbot 2470 Hongjin Qian (Renmin University of China), Xiaohe Li (Renmin University of China), Hanxun Zhong (Renmin University of China), Yu Guo (Renmin University of China), Yueyuan Ma (Renmin University of China), Yutao Zhu (Université de Montréal), Zhanliang Liu (Renmin University of China), Zhicheng Dou (Renmin University of China), Ji-Rong Wen (Renmin University of China) 
- POINTREC: A Test Collection for Narrative-driven Point of Interest Recommendation

Jafar Afzali (University of Stavanger), Aleksander Mark Drzewiecki (University of Stavanger),

Krisztian Balog (University of Stavanger)

- Seer-Dock: A General-Purpose Dockerized Scholarly Document Collection and Management Framework.

Dina Sayed (University of Basel), Mohamed Nour (Independent Researcher),

Heiko Schuldt (University of Basel)

- Select, Substitute, Search: A New Benchmark for Knowledge-Augmented

Visual Question Answering.

Aman Jain (Indian Institute of Technology Bombay),

Mayank Kothyari (Indian Institute of Technology Bombay), Vishwajeet Kumar (IBM Research),

Preethi Jyothi (Indian Institute of Technology Bombay),

Ganesh Ramakrishnan (Indian Institute of Technology Bombay),

Soumen Chakrabarti (Indian Institute of Technology Bombay)

- Simulating User Satisfaction for the Evaluation of Task-oriented Dialogue Systems

Weiwei Sun (Shandong University), Shuo Zhang (Bloomberg), Krisztian Balog (University of Stavanger),

Zhaochun Ren (Shandong University), Pengjie Ren (Shandong University),

Zhumin Chen (Shandong University), Maarten de Rijke (University of Amsterdam \& Ahold Delhaize Research)

- TripClick: The Log Files of a Large Health Web Search Engine

Navid Rekabsaz (fohannes Kepler University), Oleg Lesota (fohannes Kepler University),

Markus Schedl (fohannes Kepler University), Jon Brassey (Trip Database),

Carsten Eickhoff (Brown University)

- WTR: A Test Collection for Web Table Retrieval

Zhiyu Chen (Lehigh University), Shuo Zhang (Bloomberg), Brian D. Davison (Lehigh University)

\section{Demonstration Papers I}

- Chatty Goose: A Python Framework for Conversational Search

Edwin Zhang (University of Waterloo), Sheng-Chieh Lin (University of Waterloo),

Jheng-Hong Yang (University of Waterloo), Ronak Pradeep (University of Waterloo),

Rodrigo Nogueira (University of Waterloo), Jimmy Lin (University of Waterloo)

- DarkJargon.net: A Platform for Understanding Underground Conversation

with Latent Meaning

Dominic Seyler (University of Illinois at Urbana-Champaign),

Wei Liu (University of Illinois at Urbana-Champaign),

Yunan Zhang (University of Illinois at Urbana-Champaign),

XiaoFeng Wang (Indiana University Bloomington),

ChengXiang Zhai (University of Illinois at Urbana-Champaign)

- OpenMatch: An Open Source Library for Neu-IR Research

Zhenghao Liu (Tsinghua University), Kaitao Zhang (Tsinghua University), Chenyan Xiong (Microsoft Research),

Zhiyuan Liu (Tsinghua University), Maosong Sun (Tsinghua University)

- Precision Medicine Search for Paediatric Oncology

Bevan Koopman (CSIRO), Tracey Wright (CSIRO), Natacha Omer (Queensland Health),

Veronica McCabe (Children's Hospital Foundation), Guido Zuccon (University of Queensland)

- PYA0: A Python Toolkit for Accessible Math-Aware Search.

Wei Zhong (University of Waterloo), Jimmy Lin (University of Waterloo)

- QuARk: A GUI for Quality-Aware Ranking of Arguments.

Markus Nilles (Trier University), Lorik Dumani (Trier University), Ralf Schenkel (Trier University)

- The Information Retrieval Anthology.

Martin Potthast (Leipzig University),Sebastian Günther (Martin-Luther-Universität Halle-Wittenberg),

Janek Bevendorff (Bauhaus-Universität Weimar),

Jan Philipp Bittner (Martin-Luther-Universität Halle-Wittenberg),

Alexander Bondarenko (Martin-Luther-Universität Halle-Wittenberg),

Maik Fröbe (Martin-Luther-Universität Halle-Wittenberg), Christian Kahmann (Leipzig University),

Andreas Niekler (Leipzig University), Michael Völske (Bauhaus-Universität Weimar),

Benno Stein (Bauhaus-Universität Weimar),

Matthias Hagen (Martin-Luther-Universität Halle-Wittenberg) 


\section{Demonstration Papers II}

- A Web-based Knowledge Hub for Exploration of Multiple Research Article Collections ..... 2556 Wei Emma Zhang (The University of Adelaide), Miao Liu (Wuhan University), Alan Pallath (The University of Adelaide), Gokul Tamilventhan (The University of Adelaide)

- FedNLP: An Interpretable NLP System to Decode Federal Reserve Communications 2560 Jean Lee (The University of Sydney), Hoyoul Luis Youn (KPMG Australia), Nicholas Stevens (NRS Technology), Josiah Poon (The University of Sydney), Soyeon Caren Han (The University of Sydney)

- GeoWINE: Geolocation based Wiki, Image, News and Event Retrieval

Golsa Tahmasebzadeh (TIB - Leibniz Information Centre for Science and Technology),

Endri Kacupaj (University of Bonn),

Eric Müller-Budack (TIB - Leibniz Information Centre for Science and Technology),

Sherzod Hakimov (TIB - Leibniz Information Centre for Science and Technology), Jens Lehmann (University of Bonn \& Fraunhofer IAIS Dresden), Ralph Ewerth (TIB - Leibniz Information Centre for Science and Technology \& L3S Research Center)

- OrgBox: Supporting Cognitive and Metacognitive Activities During Exploratory Search .... 2570 Austin R. Ward (University of North Carolina at Chapel Hill), Robert Capra (University of North Carolina at Chapel Hill)

- QuTI! Quantifying Text-Image Consistency in Multimodal Documents Matthias Springstein (TIB - Leibniz Information Centre for Science and Technology), Eric Müller-Budack (TIB - Leibniz Information Centre for Science and Technology), Ralph Ewerth (TIB - Leibniz Information Centre for Science and Technology)

- Towards Trustworthiness in the Context of Explainable Search Sayantan Polley (Otto von Guericke University Magdeburg), Rashmi Raju Koparde (Otto von Guericke University Magdeburg), Akshaya Bindu Gowri (Otto von Guericke University Magdeburg), Maneendra Perera (Otto von Guericke University Magdeburg), Andreas Nuernberger (Otto von Guericke University Magdeburg)

- YASBIL: Yet Another Search Behaviour (and) Interaction Logger 2585 Nilavra Bhattacharya (The University of Texas at Austin), Jacek Gwizdka (The University of Texas at Austin)

\section{Demonstration Papers III}

- Big Brother: A Drop-In Website Interaction Logging Service Harrisen Scells (The University of Queensland), Jimmy (University of Surabaya), Guido Zuccon (The University of Queensland)

- DiffIR: Exploring Differences in Ranking Models' Behavior Kevin Martin Jose (Max Planck Institute for Informatics), Thong Nguyen (Max Planck Institute for Informatics), Sean MacAvaney (University of Glasgow), Jeffrey Dalton (University of Glasgow), Andrew Yates (Max Planck Institute for Informatics)

- Interacting with Information in Immersive Virtual Environments 2600 Austin R. Ward (University of North Carolina at Chapel Hill), Yiyin Gu (University of North Carolina at Chapel Hill), Sandeep Avula (Amazon), Praneeth Chakravarthula (University of North Carolina at Chapel Hill)

- News2PubMed: A Browser Extension for Linking Health News to Medical Literature. 2605 Jun Wang (Independent Researcher), Bei Yu (Syracuse University)

- PECAN: A Platform for Searching Chat Conversations 2610 Kunpeng Qin (The University of Queensland), Harrisen Scells (The University of Queensland), Guido Zuccon (The University of Queensland)

- Privacy-Aware Remote Information Retrieval User Experiments Logging Tool. 2615 Hanyu Li (Tsinghua University), Hongyu Lu (Tsinghua University), Songhao Huang (Tsinghua University), Weizhi Ma (Tsinghua University), Min Zhang (Tsinghua University), Yiqun Liu (University), Shaoping Ma (, Tsinghua University)

- Science2Cure: A Clinical Trial Search Prototype 2620 Maciej Rybinski (CSIRO Data61), Sarvnaz Karimi (CSIRO Data61), Aleney Khoo (CSIRO Data61) 


\section{SIRIP papers}

- Learning with Little Data: Industry Challenges and Innovations

Nikhil Rao (Amazon)

- Restoring Healthy Online Discourse by Detecting and Reducing Controversy, Misinformation, and Toxicity Online

Shiri Dori-Hacohen $(A u C o D e)$, Keen Sung $(A u C o D e)$, Jengyu Chou $(A u C o D e)$,

Julian Lustig-Gonzalez $(A u C o D e)$

- From Producer Success to Retention: A New Role of Search and Recommendation Systems on Marketplaces

Viet Ha-Thuc (Facebook Inc.), Matthew Wood (Facebook Inc.), Yunli Liu (Facebook Inc.),

Jagadeesan Sundaresan (Facebook Inc.)

- Putting the Role of Personalization into Context

Dmitri Goldenberg (Booking.com)

- SearchGCN: Powering Embedding Retrieval by Graph Convolution Networks for E-Commerce Search

Xinlin Xia (FD.com), Shang Wang (FD.com), Han Zhang (FD.com), Songlin Wang (FD.com),

Sulong Xu (FD.com), Yun Xiao (FD.com Silicon Valley Research Center),

Bo Long (JD.com \& JD.com Silicon Valley Research Center),

Wen-Yun Yang (FD.com Silicon Valley Research Center)

- AliMe Avatar: Multi-modal Content Production and Presentation

for Live-streaming E-commerce

Feng-Lin Li (DAMO Academy, Alibaba Group), Zhongzhou Zhao (DAMO Academy, Alibaba Group),

Qin Lu (DAMO Academy, Alibaba Group), Xuming Lin (DAMO Academy, Alibaba Group),

Hehong Chen (DAMO Academy, Alibaba Group), Bo Chen (DAMO Academy, Alibaba Group),

Liming Pu (DAMO Academy, Alibaba Group), Jiashuo Zhang (DAMO Academy, Alibaba Group),

Fu Sun (DAMO Academy, Alibaba Group), Xikai Liu (DAMO Academy, Alibaba Group),

Liqun Xie (DAMO Academy, Alibaba Group), Qi Huang (DAMO Academy, Alibaba Group),

Ji Zhang (DAMO Academy, Alibaba Group), Haiqing Chen (DAMO Academy, Alibaba Group)

- AliMe DA: A Data Augmentation Framework for Question Answering in Cold-start Scenarios

Guohai Xu (DAMO Academy, Alibaba Group), Yan Shao (DAMO Academy, Alibaba Group),

Chenliang Li (DAMO Academy, Alibaba Group), Feng-Lin Li (DAMO Academy, Alibaba Group),

Bin Bi (DAMO Academy, Alibaba Group), Ji Zhang (DAMO Academy, Alibaba Group),

Haiqing Chen (DAMO Academy, Alibaba Group)

- Al Based Information Retrieval System for Identifying Harmful Online

Gaming Patterns

Deepanshi Seth (Games24x7), Rukma Talwadker (Games24x7.com), Tridib Mukherjee (Games24x7),

Usama Chitapure (Games24x7), Nagesh Adiga (Games24x7), Avantika Gupta (Games24x7)

- Transformer-based Banking Products Recommender System

Davide Liu (Tsinghua University \& Genify.ai), George Philippe Farajalla (Genify.ai),

Alexandre Boulenger (Tsinghua University \& Genify.ai)

\section{Tutorials}

- Addressing Bias and Fairness in Search Systems

Ruoyuan Gao (Rutgers University), Chirag Shah (University of Washington)

- Beyond Probability Ranking Principle: Modeling the Dependencies among Documents ..... 2647 Liang Pang (Institute of Computing Technology, Chinese Academy of Sciences),

Qingyao Ai (The University of Utah), Jun Xu (Renmin University of China)

- Deep Learning on Graphs for Natural Language Processing

Lingfei Wu (JD.COM Silicon Valley Research Center), Yu Chen (Facebook AI),

Heng Ji (University of Illinois at Urbana-Champaign), Bang Liu (University of Montreal)

- Tutorial on Fairness of Machine Learning in Recommender Systems 2654 Yunqi Li (Rutgers University), Yingqiang Ge (Rutgers University), Yongfeng Zhang (Rutgers University) 
- Interactive Information Retrieval with Bandit Feedback .

Huazheng Wang (University of Virginia), Yiling Jia (University of Virginia),

Hongning Wang (University of Virginia)

- Interactive Information Retrieval: Models, Algorithms, and Evaluation

Chengxiang Zhai (University of Illinois at Urbana-Champaign),

- Pretrained Transformers for Text Ranking: BERT and Beyond

Andrew Yates (Max Planck Institute for Informatics),

Rodrigo Nogueira (University of Waterloo), Jimmy Lin (University of Waterloo)

- Reinforcement Learning for Information Retrieval

Alexander Kuhnle (Blue Prism AI Labs), Miguel Aroca-Ouellette (Blue Prism AI Labs),

Anindya Basu (Blue Prism AI Labs), Murat Sensoy (Blue Prism AI Labs),

John Reid (Blue Prism AI Labs), Dell Zhang (Blue Prism AI Labs)

- Stance Detection: Concepts, Approaches, Resources, and Outstanding Issues 2673 Dilek Küçük (TÜBİTAK MRC Energy Institute), Fazli Can (Bilkent University)

\section{Workshops}

- CSR 2021: The 1st International Workshop on Causality in Search and Recommendation .. 2677 Yongfeng Zhang (Rutgers University), Xu Chen (Renmin University of China), Yi Zhang (University of California, Santa Cruz), Xianjie Chen (Facebook AI Research)

- DRL4IR: 2nd Workshop on Deep Reinforcement Learning for Information Retrieval. 2681 Weinan Zhang (Shanghai fiao Tong University), Xiangyu Zhao (Michigan State University), Li Zhao (Microsoft Research), Dawei Yin (Baidu), Grace Hui Yang (Georgetown University)

- ECOM'21: The SIGIR 2021 Workshop on eCommerce 2685 Surya Kallumadi (Lowe's Companies, inc.), Tracy Holloway King (Adobe), Shervin Malmasi (Amazon.com, Inc.), Maarten de Rijke (University of Amsterdam \& Ahold Delhaize)

- IR for Children 2000-2020: Where Are We Now? 2689 Theo Huibers (University of Twente), Monica Landoni (Università della Svizzera Italiana), Emiliana Murgia (Università degli Studi di Milano-Bicocca), Maria Soledad Pera (PIReT - Boise State University)

- 2nd Workshop on Patent Text Mining and Semantic Technologies (PatentSemTech2021)... 2693 Ralf Krestel (Hasso Plattner Institute, University of Potsdam), Hidir Aras (FIZ Karlsruhe), Linda Andersson (Artificial Researcher IT GmbH), Florina Piroi (Data Science Studio), Allan Hanbury (TU Wien), Dean Alderucci (Carnegie Mellon University)

- Sim4IR: The SIGIR 2021 Workshop on Simulation for Information Retrieval Evaluation .... 2697 Krisztian Balog (University of Stavanger), David Maxwell (Delft University of Technology), Paul Thomas (Microsoft), Shuo Zhang (Bloomberg)

\section{Doctoral Consortium}

- Bridge the Gap: Industrial Data Pipeline oriented Recommendation Enhancement 2699 Jie Dai (Xidian University)

- Embedding Formulae and Text for Improved Math Retrieval. 2700 Behrooz Mansouri (Rochester Institute of Technology)

- Enabling Performance Prediction in Information Retrieval Evaluation 2701 Guglielmo Faggioli (University of Padova)

- Estimating the Reliability of Health-related Search Results 2702 Marcos Fernández-Pichel (University of Santiago de Compostela)

- HONto: A Bottom-Up Knowledge Base from Textbooks for Recommending Contextually Relevant Documents Sabine Wehnert (Georg Eckert Institute Leibniz Institute for International Textbook Research \& Otto von Guericke University Magdeburg)

- Improving Deep Learning based Multi-document Summarization through Linguistic Knowledge Congbo Ma (The University of Adelaide) 
- Interpretable Document Representations for Fast and Accurate Retrieval of Mathematical Information.

Vít Novotný (Masaryk University)

- New Perspectives to Query Performance Prediction Evaluation Oleg Zendel (RMIT University),

- Semi-automatic Labelling of Scientific Articles using Deep Learning to Enlarge Benchmark Data for Scientific Summarization Alaa El-Ebshihy (TU Wien)

- Transfer Learning for the Multilingual and Multi-Domain Classification of Messages Relating to Crises

Cinthia Sánchez (Universidad de Chile)

- Understanding How Algorithmic and Cognitive Biases in Web Search Affect User Attitudes on Debated Topics Tim Draws (Delft University of Technology)

Author Index 2710 


\title{
ACM SIGIR 2021 Conference Organization
}

\author{
General Chairs: Fernando Diaz (Google) \\ Chirag Shah (University of Washington)
}

Torsten Suel (New York University)

Program Chairs: Pablo Castells (Universidad Autónoma de Madrid, Amazon)

Rosie Jones (Spotify)

Tetsuya Sakai (Waseda University)

Short Paper Chairs: Hui Fang (University of Delaware)

Claudia Hauff (Technische Universiteit Delft)

Ruihua Song (Renmin University of China)

Perspectives Paper Chairs: Fernando Diaz (Google)

Chirag Shah (University of Washington)

Resource Paper Chairs: Yiqun Liu (Tsinghua University)

Ian Soboroff (National Institute of Standards and Technology)

Demo Chairs: Miguel Martínez (Signal AI)

Milad Shokouhi (Microsoft)

Tutorial Chairs: Sengor Altingovde (Middle East Technical University)

Rodrygo Santos (Universidade Federal de Minas Gerais)

Doctoral Consortium Chairs: Hiroaki Ohshima (University of Hyogo)

Bárbara Poblete (University of Chile)

Workshop Chairs: Abdigani Dirye (Amazon)

Jiafeng Guo (Chinese Academy of Sciences)

SIRIP Chairs: Hema Raghavan (LinkedIn)

Rishabh Mehrotra (Spotify)

Karthik Subbian (Amazon)

Proceedings Chairs: Alejandro Bellogín (Universidad Autónoma de Madrid)

Masaharu Yoshioka (Hokkaido University)

Registration Chair: Jiqun Liu (University of Oklahoma)

Virtual Chairs: Bhaskar Mitra (Microsoft)

Jian-Yun Nie (Université de Montréal)

Diversity and Inclusion Chairs: Bárbara Poblete (SIGIR Representative, University of Chile)

Hussein Suleman (SIGIR Representative, University of Cape Town)

Suzan Verberne (SIGIR Representative, Universiteit Leiden)

Bhaskar Mitra (DEI Liaison, Microsoft)

Sponsorship Chairs: Charlie Clarke (University of Waterloo)

Zhaochun Ren (Shandong University) 
Best Paper Chairs: Alistair Moffat (The University of Melbourne)

Emine Yilmaz (University College London)

Social Media Chair: Johanne Trippas (The University of Melbourne)

Webmaster: Matthew Mitsui (VidIQ) 


\section{Senior Program Committee}

Eytan Adar (University of Michigan)

Omar Alonso (Instacart)

Javed Aslam (Northeastern University)

Ahmed Hassan Awadallah (Microsoft)

Krisztian Balog (University of Stavanger)

Hannah Bast (University of Freiburg)

Nicholas Belkin (Rutgers University)

Michael Bendersky (Google)

Klaus Berberich (Saarbruecken University of Applied Sciences (htw saar))

Asia J. Biega (Max Planck Institute for Security and Privacy)

Robert Capra (The University of North Carolina at Chapel Hill)

David Carmel (Amazon)

Ben Carterette (Spotify)

Carlos Castillo (Universitat Pompeu Fabra)

James Caverlee (Texas A\&M University)

Yi Chang (Filin University)

Tat-Seng Chua (National University of Singapore)

Charles Clarke (University of Waterloo)

Matt Crane (Facebook)

Nick Craswell (Microsoft)

Bruce Croft (University of Massachusetts Amherst)

Shane Culpepper (RMIT University)

Jeffrey Dalton (University of Glasgow)

Brian Davison (Lehigh University)

Maarten de Rijke (University of Amsterdam, Ahold

Delhaize Research)

Gianluca Demartini (The University of Queensland)

Tom Diethe (Amazon)

Laura Dietz (University of New Hampshire)

Zhicheng Dou (Renmin University of China)

Carsten Eickhoff (Brown University)

Yi Fang (Santa Clara University)

Nicola Ferro (University of Padova)

Edward Fox (Virginia Tech)

Norbert Fuhr (University of Duisburg-Essen)

Eric Gaussier (LIG-UfF)

Lorraine Goeuriot (Université Grenoble Alpes)

Julio Gonzalo (UNED)

Ido Guy (eBay Research)
Donna Harman (NIST)

Max Harper (Amazon)

Djoerd Hiemstra (Radboud University)

Jeff Huang (Brown University)

Hideo Joho (University of Tsukuba)

Gareth Jones (Dublin City University)

Jaap Kamps (University of Amsterdam)

Min-Yen Kan (National University of Singapore)

Noriko Kando (National Institute of Informatics)

Jussi Karlgren (Spotify)

Makoto P. Kato (University of Tsukuba)

Gabriela Kazai (Microsoft)

Yehuda Koren (Google)

Udo Kruschwitz (University of Regensburg)

Oren Kurland (Technion, Israel Institute of Technology)

Wai Lam (The Chinese University of Hong Kong)

Matthew Lease (The University of Texas at Austin)

Jochen L. Leidner (Refinitiv Labs and University of Sheffield)

Hang Li (Bytedance AI Lab)

Jimmy Lin (University of Waterloo)

Yiqun Liu (Tsinghua University)

Yoelle Maarek (Amazon Research)

Craig Macdonald (University of Glasgow)

R. Manmatha (University of Massachusetts Amherst)

Qiaozhu Mei (University of Michigan)

Tao Mei (fD.COM)

Donald Metzler (Google)

Bhaskar Mitra (Microsoft)

Alistair Moffat (The University of Melbourne)

Alessandro Moschitti (Amazon)

Yashar Moshfeghi (University of Strathclyde)

Josiane Mothe (Institut de Recherche en Informatique de Toulouse)

Isabelle Moulinier (Capital One)

Henning Müller (HES-SO)

Marc Najork (Google)

Wolfgang Nejdl (L3S and University of Hannover)

Jian-Yun Nie (University de Montreal)

Douglas Oard (University of Maryland)

Iadh Ounis (University of Glasgow) 
Denis Parra (Pontificia Universidad Catolica de Chile)

Maria Soledad Pera (Boise State University)

Raffaele Perego (ISTI-CNR)

Benjamin Piwowarski (CNRS, University Pierre et Marie Curie)

Kira Radinsky (eBay)

Filip Radlinski (Google)

Fiana Raiber (Yahoo Research)

Berthier Ribeiro-Neto (Google Engineering Belo Horizonte, UFMG)

Paolo Rosso (Universitat Politècnica de València)

Ian Ruthven (University of Strathclyde)

Fabrizio Silvestri (Facebook)

Mark Smucker (University of Waterloo)

Aixin Sun (Nanyang Technological University)

Christoph Trattner (University of Bergen)

Andrew Trotman (University of Otago)

Christophe Van Gysel (Apple Inc.)
Suzan Verberne (LIACS, Leiden University)

Ellen Voorhees (NIST)

Xuanhui Wang (Google)

Gerhard Weikum (Max Planck Institute for Informatics)

Ji-Rong Wen (Renmin University of China)

Ryen White (Microsoft)

Jun Xu (Renmin University of China)

Rui Yan (Renmin University of China)

Grace Hui Yang (Georgetown University)

Dawei Yin (FD.com)

Hamed Zamani (Microsoft)

Min Zhang (Tsinghua University)

Xin Zhao (Renmin University of China)

Imed Zitouni (Google)

Justin Zobel (The University of Melbourne)

Guido Zuccon (The University of Queensland) 


\section{Program Committee}

Panagiotis Adamopoulos (Emory University)

Eytan Adar (University of Michigan)

Wasi Ahmad (University of California)

Qingyao Ai (University of Utah)

Wei Ai (University of Maryland)

Mohammad Akbari (University College London)

Ahmet Aker (University of Duisburg Essen)

Elif Aktolga (Apple Inc.)

Dyaa Albakour (Signal AI)

Mohammad Aliannejadi (University of Amsterdam)

Omar Alonso (Instacart)

Ismail Sengor Altingovde (Middle East Technical University)

Giuseppe Amato (ISTI-CNR)

Jesse Anderton (Spotify)

Avi Arampatzis (Democritus University of Thrace)

Ioannis Arapakis (Telefonica Research)

Jaime Arguello (The University of North Carolina at Chapel Hill)

Azin Ashkan (Google)

Javed Aslam (Northeastern University)

Renato Assuncao (UFMG)

Ahmed Hassan Awadallah (Microsoft)

Hosein Azarbonyad (University of Amsterdam)

Maryam Aziz (Spotify)

Leif Azzopardi (University of Strathclyde)

Ebrahim Bagheri (Ryerson University)

Ashraf Bah Rabiou (University of Delaware)

Ting Bai (Beijing University of Posts and

Telecommunications)

Xiao Bai (Yahoo Research)

Krisztian Balog (University of Stavanger)

Jie Bao (fD Digits)

Zhifeng Bao (RMIT University)

Alberto Barrón-Cedeño (University of Bologna)

Hannah Bast (University of Freiburg)

Srikanta Bedathur (IIT Delhi)

Nicholas Belkin (Rutgers University)

Alejandro Bellogín (Universidad Autónoma de Madrid)

Patrice Bellot (Aix-Marseille Université - CNRS (LSIS))

Michael Bendersky (Google)

Rodger Benham (RMIT University)
Klaus Berberich (Saarbruecken University of Applied Sciences (htw saar))

Rafael Berlanga (Universitat Jaume I)

Asia J. Biega (Max Planck Institute for Security and Privacy)

Bodo Billerbeck (Bing)

Ludovico Boratto (Eurecat - Centre Tecnòlogic de Catalunya)

Ilaria Bordino (UniCredit R\&D)

Gloria Bordogna (National Research Council of Italy CNR)

Hugues Bouchard (Spotify)

Martin Braschler (ZHAW)

Pavel Braslavski (Ural Federal University)

Sebastian Bruch (National Institutes of Health)

George Buchanan (The University of Melbourne)

Robin Burke (University of Colorado)

Fidel Cacheda (Universidade da Coruña)

Fei Cai (National University of Defense Technology)

Jamie Callan (Carnegie Mellon University)

Arthur Câmara (Delft University of Technology)

B. Barla Cambazoglu (RMIT University)

Fazli Can (Bilkent University)

Iván Cantador (Universidad Autónoma de Madrid)

Xin Cao (The University of New South Wales)

Robert Capra (The University of North Carolina at Chapel Hill)

David Carmel (Amazon)

Jorge Carrillo-De-Albornoz (Universidad Nacional de Educacion a Distancia)

Ben Carterette (Spotify)

James Caverlee (Texas A\&M University)

Michelangelo Ceci (Universita degli Studi di Bari)

Aniket Chakrabarti (Amazon)

Tanmoy Chakraborty (IIIT-Delhi)

Zhangming Chan (Peking University)

Praveen Chandar (Spotify Research)

Kevin Chang (University of Illinois at UrbanaChampaign)

Chen Chen (Arizona State University)

Hsin-Hsi Chen (National Taiwan University)

Huiyuan Chen (Visa Research) 
Jia Chen (Carnegie Mellon University)

Jian Chen (South China University of Technology)

Jiecao Chen (Google LLC)

Li Chen (Hong Kong Baptist University)

Liang Chen (Sun Yat-Sen University)

Ping Chen (UMB)

Ruey-Cheng Chen (SEEK Ltd)

Tao Chen (Google AI)

Yi Chen (New Jersey Institute of Technology)

Zhengzhang Chen (NEC Laboratories America)

Zhumin Chen (Shandong University)

Wei Cheng (NEC Labs America)

Zhiyong Cheng (Shandong Artificial Intelligence Institute)

Max Chevalier (IRIT)

Jean-Pierre Chevallet (Grenoble Alpes University)

Adrian-Gabriel Chifu (Aix Marseille Univ, Université de Toulon, CNRS, LIS)

Evangelia Christakopoulou (University of Minnesota)

Tat-Seng Chua (National University of Singapore)

Charles Clarke (University of Waterloo)

Anne Clifton (Spotify)

Michael Cole (LexisNexis)

Humberto Corona (University College Dublin)

Matt Crane (Facebook)

Nick Craswell (Microsoft)

Anita Crescenzi (The University of North Carolina at Chapel Hill)

Bruce Croft (University of Massachusetts Amherst)

Shane Culpepper (RMIT University)

Tonya Custis (Autodesk)

Zhuyun Dai (Google)

Jeffrey Dalton (University of Glasgow)

Elizabeth M. Daly (IBM)

Ariyam Das (University of California)

Brian Davison (Lehigh University)

Edleno Silva De Moura (Universidade Federal do Amazonas)

Maarten de Rijke (University of Amsterdam, Ahold Delhaize Research)

Arjen de Vries (Radboud University)

Yashar Deldjoo (Polytechnic University of Bari)

Harm Delva (Ghent University - imec)

Gianluca Demartini (The University of Queensland)
Elena Demidova (Bonn University)

Dong Deng (Rutgers University)

Hongbo Deng (Google)

Giorgio Maria Di Nunzio (University of Padua)

Tom Diethe (Amazon)

Laura Dietz (University of New Hampshire)

Stefan Dietze (GESIS - Leibniz Institute for the Social Sciences)

Fernando Díez (Universidad Autónoma de Madrid)

Djellel Difallah (NYU)

B. Taner Dincer (University of Mugla)

Steven Ding (McGill University)

Nemanja Djuric (Aurora Innovation Inc.)

Zhicheng Dou (Renmin University of China)

Carsten Eickhoff (Brown University)

Michael Ekstrand (Boise State University)

Mehdi Elahi (University of Bergen)

Tamer Elsayed (Qatar University)

Gonenc Ercan (Hacettepe University)

Liana Ermakova (Université de Bretagne Occidentale)

Yi Fang (Santa Clara University)

Hossein Fani (University of Windsor)

Fuli Feng (National University of Singapore)

Kaiyu Feng (Nanyang Technological University)

Yue Feng (UCL)

Ignacio Fernández (Amazon)

Diego Fernández Iglesias

Juan Manuel Fernández-Luna (University of Granada)

Nicola Ferro (University of Padova)

Besnik Fetahu (Amazon)

Simone Filice (Amazon)

Adam Fourney (Microsoft)

Sébastien Fournier (LSIS)

Edward Fox (Virginia Tech)

Yanjie Fu (University of Central Florida)

Norbert Fuhr (University of Duisburg-Essen)

Patrick Gallinari (LIP6 - University of Paris 6)

Petra Galuščáková (University of Maryland)

Iftah Gamzu (Amazon)

Debasis Ganguly (University of Glasgow)

Shen Gao (Peking University)

Wei Gao (Singapore Management University)

Yang Gao (The University of Texas at Dallas) 
Yunjun Gao (Zhejiang University)

Jean Garcia-Gathright (Spotify)

Dario Garigliotti

Eric Gaussier (LIG-UfF)

Hancheng Ge (Amazon)

Saptarshi Ghosh (Indian Institute of Technology Kharagpur)

Anastasia Giachanou (Universitat Politècnica de València)

Boris Glavic (Illinois Institute of Technology)

Lorraine Goeuriot (Université Grenoble Alpes)

Marcos Goncalves (Federal University of Minas Gerais)

Julio Gonzalo (UNED)

Gregory Goren (eBay)

Priya Govindan (Pinterest)

Pawan Goyal (IIT Kharagpur)

Quanquan Gu (University of California)

Yupeng Gu (Pinterest)

Ziyu Guan (Xidian Universitiy)

Francesco Guerra (Università di Modena e Reggio Emilia)

Francesco Gullo (UniCredit)

Guibing Guo (Northeastern University)

Tao Guo (Nanyang Technological University)

Parth Gupta (Amazon)

Ido Guy (eBay Research)

Hamed Haddadi (Imperial College London)

Zhen Hai (Institute for Infocomm Research, $A{ }^{*}$ STAR)

Nissim Halabi (Amazon)

Martin Halvey (University of Strathclyde)

Lei Han (The University of Queensland)

Shuchu Han (StellarCyber.ai)

Allan Hanbury (Vienna University of Technology)

Mengyue Hang (Purdue University)

Preben Hansen (Stockholm University)

Max Harper (Amazon)

Morgan Harvey (University of Sheffield)

Helia Hashemi (University of Massachusetts Amherst)

Seyyed Hadi Hashemi (University of Amsterdam)

Ben He (University of Chinese Academy of Sciences)

Daqing He (University of Pittsburgh)

Jingrui He (University of Illinois at Urbana-Champaign)

Jiyin He (Signal)

Qi He (LinkedIn)
Xiangnan He (University of Science and Technology of China)

Djoerd Hiemstra (Radboud University)

Tuan-Anh Hoang (L3S Research Center, Leibniz University of Hanover)

Orland Hoeber (University of Regina)

Liangjie Hong (LinkedIn Inc.)

Richang Hong (Hefei University of Technology)

Frank Hopfgartner (The University of Sheffield)

Hsun-Ping Hsieh (National Cheng Kung University)

Diane $\mathrm{Hu}$ (Etsy)

Xia Ben $\mathrm{Hu}$ (Texas A\&M University)

Yifan $\mathrm{Hu}$ (Yahoo!)

Chao Huang (University of Notre Dame)

Jeff Huang (Brown University)

Jimmy Huang (York University)

Xin Huang (Hong Kong Baptist University)

Xuanjing Huang (Fudan University)

Zi Huang (The University of Queensland)

Juan F. Huete (University of Granada)

Kai Hui (Amazon)

Muhammad Imran (Qatar Computing Research Institute)

Amir Ingber (Amazon Research)

Radu Tudor Ionescu (University of Bucharest)

Sagar Jain (Google)

Shoaib Jameel (University of Essex)

Jiepu Jiang (University of Wisconsin-Madison)

Minhao Jiang (Hong Kong University of Science and Technology)

Peng Jiang (Kwai Inc.)

Xiaolong Jin (Chinese Academy of Sciences)

How Jing (LinkedIn)

Hideo Joho (University of Tsukuba)

Gareth Jones (Dublin City University)

Joemon Jose (University of Glasgow)

Parisa Kaghazgaran (Amazon)

Jaap Kamps (University of Amsterdam)

Min-Yen Kan (National University of Singapore)

Noriko Kando (National Institute of Informatics)

Nattiya Kanhabua (Upwork)

Evangelos Kanoulas (University of Amsterdam)

Jussi Karlgren (Spotify)

Santu Karmaker (Auburn University)

Makoto P. Kato (University of Tsukuba) 
Markus Kattenbeck (TU Wien)

Gabriela Kazai (Microsoft)

Jaana Kekäläinen (University of Tampere)

Jinha Kim (Oracle Labs)

Sang-Wook Kim (Hanyang University)

Yubin Kim (UPMC Enterprises)

Deguang Kong (Google)

Weize Kong (Google)

Arnd Christian König (Microsoft)

Bevan Koopman (CSIRO)

Alexander Kotov (Wayne State University)

Pigi Kouki (relationalAI)

Udo Kruschwitz (University of Regensburg)

Oren Kurland (Technion, Israel Institute of Technology)

Anisio Lacerda (Centro Federal de Educação Tecnológica de Minas Gerais)

Wai Lam (The Chinese University of Hong Kong)

Yanyan Lan (ICT)

Ni Lao (Apple)

Léa Laporte (INSA Lyon - LIRIS)

Gal Lavee (Microsoft)

Matthew Lease (The University of Texas at Austin)

Kyumin Lee (Worcester Polytechnic Institute)

Roy Ka-Wei Lee (University of Saskatchewan)

Wenqiang Lei (National University of Singapore)

Jochen L. Leidner (Refinitiv Labs and University of Sheffield)

Luis A. Leiva (University of Luxembourg)

Daniel Lemire (LICEF Research Center, Université du Québec)

Ran Levy (Amazon)

Cheng Li (Google)

Cheng Li (CodaMetrix LLC)

Chengkai Li (University of Texas at Arlington)

Cheng-Te Li (National Cheng Kung University)

Chenliang Li (Wuhan University)

Guoliang Li (Tsinghua University)

Hang Li (Bytedance AI Lab)

Jianxin Li (Deakin University)

Jundong Li (University of Virginia)

Liangda Li (SfTU)

Liangyue Li (Arizona State University)

Piji Li (Tencent AI Lab)

Rong-Hua Li (Shenzhen University)
Ruoyu Li (UT Arlington)

Shuai Li (Cambridge University)

Xian Li (Amazon)

Ximing Li (Filin University)

Xin Li (Sogou Inc.)

Xin Li (Beijing Institute of Technology)

Yaliang Li (Alibaba Group)

Yong Li (Tsinghua University)

Zeyu Li (University of California)

Panagiotis Liakos (NKUA)

Defu Lian (University of Science and Technology of China)

Xiang Lian (Kent State University)

Hongwei Liang (Microsoft)

Jiongqian Liang (Google)

Shangsong Liang (Sun Yat-sen University)

Lizi Liao (National University of Singapore)

Alexander Libov (Amazon)

Matteo Lissandrini (Aalborg University)

Bin Liu (West Virginia University)

Chang Liu (Peking University)

Chuanren Liu (University of Tennessee)

Honglei Liu (University of California)

Jinfei Liu (Emory University)

Jingjing Liu (M.D. Anderson Cancer Center)

Jiqun Liu (University of Oklahoma)

Kang Liu (Chinese Academy of Sciences)

Lin Liu (University of South Australia)

Mengwen Liu (Drexel University)

Qi Liu (University of Science and Technology of China)

Tingwen Liu (Chinese Academy of Sciences)

Wei Liu (Tencent AI Lab)

Xiaodong Liu (Microsoft)

Xiaozhong Liu (Indiana University Bloomington)

Xuanzhe Liu (Peking University)

Yanchi Liu (Rutgers University)

Yang Liu (Wilfrid Laurier University)

Yiqun Liu (Tsinghua University)

Andreas Lommatzsch (TU Berlin)

David Losada (University of Santiago de Compostela)

Charlie Louis (Amazon)

Xiaolu Lu (Microsoft)

Cheng Luo (Tsinghua University) 
Jiyun Luo (Pinterest)

Ping Luo (University of Chinese Academy of Sciences)

Yuanhua Lv (Microsoft)

Lin Ma (Tencent AI Lab)

Weizhi Ma (Tsinghua University)

Yoelle Maarek (Amazon Research)

Craig Macdonald (University of Glasgow)

Andrew Macfarlane (City - University of London)

Joel Mackenzie (The University of Melbourne)

Maria Maistro (University of Copenhagen)

Tomohiro Manabe (Yahoo Japan Corporation)

R. Manmatha (University of Massachusetts Amherst)

Jiaxin Mao (Renmin University of China)

Xian-Ling Mao (Beijing Institute of Technology)

Mirko Marras (École Polytechnique Fédérale de Lausanne - EPFL)

Flavio Martins (Universidade NOVA de Lisboa)

Yosi Mass (IBM Haifa Research Lab)

Richard Mccreadie (University of Glasgow)

Graham Mcdonald (University of Glasgow)

Paul McNamee (Fohns Hopkins University)

Parth Mehta (IRSI)

Qiaozhu Mei (University of Michigan)

Tao Mei (fD.COM)

Florian Meier (Aalborg University)

Edgar Meij (Bloomberg L.P.)

Wagner Meira Jr. (Universidade Federal de Minas Gerais)

Ida Mele (IASI-CNR)

Zaiqiao Meng (University of Glasgow)

Donald Metzler (Google)

Bhaskar Mitra (Microsoft)

Stefano Mizzaro (University of Udine)

Alistair Moffat (The University of Melbourne)

Boughanem Mohand (IRIT University Paul Sabatier Toulouse)

Ali Montazeralghaem (University of Massachusetts)

Manuel Montes-Y-Gómez (Instituto Nacional

de Astrofísica, Óptica y Electrónica)

Felipe Moraes (Delft University of Technology)

Jose Moreno (IRIT, UPS)

Alessandro Moschitti (Amazon)

Yashar Moshfeghi (University of Strathclyde)

Josiane Mothe (Institut de Recherche en Informatique de Toulouse)
Isabelle Moulinier (Capital One)

Ahmed Mourad (The University of Queensland)

Philippe Mulhem (LIG-CNRS)

Henning Müller (HES-SO)

Vanessa Murdock (Amazon)

Seung-Hoon Na (Chonbuk National University)

Parth Nagarkar (New Mexico State University)

Marc Najork (Google)

Preslav Nakov (Qatar Computing Research Institute, $H B K U)$

Franco Maria Nardini (ISTI-CNR)

Fedelucio Narducci (Politecnico di Bari)

Shahrzad Naseri (University of Massachusetts Amherst)

Wolfgang Nejdl (L3S and University of Hannover)

Jian-Yun Nie (University de Montreal)

Liqiang Nie (Shandong University)

Zhi Nie (Samsung Research America)

Xia Ning (The Ohio State University)

Alexandros Ntoulas (National and Kapodistrian University of Athens)

Douglas Oard (University of Maryland)

Neil O'Hare (Yahoo Research)

Harrie Oosterhuis (Radboud University)

Vincent Oria (NFIT)

Iadh Ounis (University of Glasgow)

Jiaul Paik (IIT Kharagpur)

Liang Pang (ICT)

Manos Papagelis (York University)

Haris Papageorgiou (Institute for Language and Speech Processing)

Evangelos Papalexakis (University of California Riverside)

Aasish Pappu (Spotify)

Javier Parapar (University of A Coruña)

Dae Hoon Park (Yahoo Research)

Denis Parra (Pontificia Universidad Católica de Chile)

Rama Kumar Pasumarthi (Google)

Saurabh Paul (Kohl's Department Stores)

Virgil Pavlu (Northeastern Univ)

Jan Pedersen (eBay)

Jian Pei (Simon Fraser University)

Konstantinos Pelechrinis (University of Pittsburgh)

Gustavo Penha (Delft University of Technology)

Maria Soledad Pera (Boise State University) 
Raffaele Perego (ISTI-CNR)

Fabio Petroni (Facebook AI Research)

Hai Phan (New Jersey Institute of Technology)

Lighton Phiri (University of Zambia)

Giulio Ermanno Pibiri (ISTI-CNR)

Fabio Pinelli (IMT Lucca)

Benjamin Piwowarski (CNRS, University Pierre et Marie Curie)

Vassilis Plachouras (Facebook)

Laura Plaza (Universidad Nacional de Educación a Distancia)

Animesh Prasad (Amazon Alexa)

Jianzhong Qi (The University of Melbourne)

Buyue Qian (Xi'an fiaotong University)

Zhen Qin (Google)

Kira Radinsky (eBay)

Filip Radlinski (Google)

Davood Rafiei (University of Alberta)

Razieh Rahimi (University of Massachusetts Amherst)

Fiana Raiber (Yahoo Research)

Maya Ramanath (IIT Delhi)

Ashwani Rao (Amazon)

Jinfeng Rao (University of Maryland)

Lida Rashidi (The University of Melbourne)

Edie Rasmussen (The University of British Columbia)

Sravana Reddy (Spotify)

Pengjie Ren (Shandong University)

Yongli Ren (RMIT University)

Zhaochun Ren (Shandong University)

Chiara Renso (ISTI-CNR)

Berthier Ribeiro-Neto (Google Engineering Belo

Horizonte, UFMG)

Thomas Roelleke (Queen Mary University of London)

Kevin Roitero (University of Udine)

Haggai Roitman (eBay Research)

Paolo Rosso (Universitat Politècnica de València)

Dwaipayan Roy (GESIS - Leibniz Institute for the Social Sciences)

Natali Ruchansky (Netflix)

Tuukka Ruotsalo (University of Helsinki and University of Copenhagen)

Ian Ruthven (University of Strathclyde)

Sourav S Bhowmick (Nanyang Technological University)

Altigran S. Da Silva (Universidade Federal do Amazonas)
Rishiraj Saha Roy (Max Planck Institute for Informatics)

Ariadna Sanchez (Amazon)

Eric Sanjuan (Université d'Avignon)

Rodrygo Santos (Universidade Federal de Minas Gerais)

Javier Sanz-Cruzado (Universidad Autónoma de Madrid)

Maria Luisa Sapino (Universita' di Torino)

Harrisen Scells (The University of Queensland)

Ralf Schenkel (Trier University)

Steven Schockaert (Cardiff University)

Azadeh Shakery (University of Tehran)

Xindi Shang (National University of Singapore)

Eilon Sheetrit (Technion - Israel Institute of Technology)

Huawei Shen (Chinese Academy of Sciences)

Yue Shi (Facebook)

Kyuseok Shim (Seoul National University)

Gianmaria Silvello (University of Padua)

Fabrizio Silvestri (Facebook)

Jakob Grue Simonsen (University of Copenhagen)

Jaspreet Singh (L3S Research Centre)

Catherine Smith (Kent State University)

Mark Smucker (University of Waterloo)

Dawei Song (The Open University)

Shaoxu Song (Tsinghua University)

Xuemeng Song (National University of Singapore)

Laure Soulier (Sorbonne Université-LIP6)

Damiano Spina (RMIT University)

Efstathios Stamatatos (University of the Aegean)

Kostas Stefanidis (Tampere University)

Kazunari Sugiyama (Kyoto University)

Aixin Sun (Nanyang Technological University)

Fei Sun (Alibaba Group)

Hailong Sun (Beihang University)

Leilei Sun (Beihang University)

S.M.M. Tahaghoghi (Facebook)

Keishi Tajima (Kyoto University)

Lynda Tamine (IRIT)

Jiliang Tang (Michigan State University)

Jinhui Tang (Nanjing University of Science and Technology)

Jintao Tang (School of Computer, National University of Defense Technology)

Ruiming Tang (Huawei)

Zhiwen Tang (Georgetown University) 
Junichi Tatemura (Google)

Yi Tay (Google)

Jin Ting

Riku Togashi (Waseda University, Mercari Inc.)

Gabriele Tolomei (Sapienza University of Rome)

Nicola Tonellotto (University of Pisa)

Hanghang Tong (University of Illinois at UrbanaChampaign)

Yongxin Tong (Beihang University)

Ismail Toroslu (Middle East Technical Univ.)

Salvatore Trani (ISTI-CNR)

Christoph Trattner (University of Bergen)

Andrew Trotman (University of Otago)

Manos Tsagkias (904Labs)

Ming-Feng Tsai (National Chengchi University)

Theodora Tsikrika (Information Technologies Institute, CERTH)

Kosetsu Tsukuda (National Institute of Advanced Industrial Science and Technology (AIST))

Md Zia Ullah (CNRS)

Kazutoshi Umemoto (The University of Tokyo)

Julián Urbano (Delft University of Technology)

Christophe Van Gysel (Apple Inc.)

Olivier Van Laere (Apple Inc.)

Ali Vardasbi (University of Amsterdam, ILPS)

Theodoros Vasiloudis (Amazon)

Nikhita Vedula (Amazon)

Rossano Venturini (Università di Pisa)

Suzan Verberne (LIACS, Leiden University)

Karin Verspoor (RMIT University)

Vishwa Vinay (Adobe Research)

Ellen Voorhees (NIST)

Henning Wachsmuth (Paderborn University)

Huaiyu Wan (Beijing fiaotong University)

Bin Wang (Chinese Academy of Sciences)

Chong Wang (S\&P Global)

Chuan-Ju Wang (Academia Sinica)

Hongning Wang (University of Virginia)

Hongzhi Wang (Harbin Institute of Technology)

Jianguo Wang (Purdue University)

Jianyong Wang (Tsinghua University)

Jingang Wang (Meituan-Dianping Group)

Lidan Wang (University of Maryland)

Meng Wang (Hefei University of Technology)
Ning Wang (Beijing fiaotong University)

Shuaiqiang Wang (Baidu Inc.)

Suhang Wang (Arizona State University)

Tong Wang (Amazon)

Wei Wang (The University of New South Wales)

Weiqing Wang (Monash University)

Xiang Wang (National University of Singapore)

Xuanhui Wang (Google)

Yue Wang (University of North Carolina at Chapel Hill)

Ziqi Wang (Facebook)

Gerhard Weikum (Max Planck Institute for Informatics)

Zeyi Wen (The University of Western Australia)

Ryen White (Microsoft)

Kyle Williams (Microsoft)

Ran Wolff (Amazon)

Fangzhao Wu (Microsoft Research Asia)

Liang Wu (Airbnb Inc.)

Shan-Hung Wu (National Tsing Hua University)

Wentao Wu (Microsoft)

Yang $\mathrm{Wu}$ (Facebook)

Yikun Xian (Rutgers University)

Keli Xiao (Stony Brook University)

Yanghua Xiao (Fudan University)

Sihong Xie (Lehigh University)

Xiaohui Xie (Tsinghua University)

Xike Xie (University of Science and Technology of China)

Haoyi Xiong (Missouri University of Science and Technology)

Chen Xiuying (Wuhan University)

Jian Xu (Alibaba Group)

Jingwei Xu (Nanjing University)

Jun Xu (Renmin University of China)

Takehiro Yamamoto (University of Hyogo)

Cong Yan (Microsoft Research)

Rui Yan (Renmin University of China)

De-Nian Yang (Academia Sinica)

Grace Hui Yang (Georgetown University)

Jie Yang (Delft University of Technology)

Liu Yang (Google, University of Massachusetts Amherst)

Tao Yang (University of California at Santa Barbara)

Yu Yang (City University of Hong Kong)

Yuan Yao (Nanjing University)

Dawei Yin (FD.com) 
Hongzhi Yin (The University of Queensland)

Fan Yixing (ict)

Guoxian Yu (Shandong University)

Hai-Tao Yu (University of Tsukuba)

Wenchao Yu (University of California)

Shuhan Yuan (Utah State University)

Ye Yuan (NEU)

Reza Zafarani (Syracuse University)

Hamed Zamani (Microsoft)

Richard Zanibbi (Rochester Institute of Technology)

Fattane Zarrinkalam (Ryerson University)

Meike Zehlike (MPI Software Systems)

Zhaohao Zeng (Waseda University)

Dan Zhang (Facebook)

Fan Zhang (Tsinghua University)

Hainan Zhang (Chinese Academy of Sciences)

Hanwang Zhang (Nanyang Technological University)

Jiawei Zhang (Florida State University)

Min Zhang (Tsinghua University)

Mingyang Zhang (Google)

Peng Zhang (Tianjin University)

Qi Zhang (Fudan University)

Richong Zhang (Beihang University)

Rongting Zhang (Amazon)

Ruqing Zhang (Chinese Academy of Sciences)

Shuo Zhang (Bloomberg)

Wei Zhang (East China Normal University)

Weinan Zhang (Harbin Institute of Technology)

Wenjie Zhang (The University of New South Wales)
Xuchao Zhang (Virginia Tech)

Xuyun Zhang (Macquarie University)

Ying Zhang (Nankai University)

Yongfeng Zhang (Rutgers University)

Jiashu Zhao (Wilfrid Laurier University)

Kaiqi Zhao (University of Auckland)

Le Zhao

Qian Zhao (Bloomberg L.P.)

Xin Zhao (Renmin University of China)

Xueliang Zhao (Peking University)

Ying Zhao (Tsinghua University)

Zhou Zhao (Zhejiang University)

Cheng Zheng (Facebook Inc)

Yong Zheng (Illinois Institute of Technology)

Yudian Zheng (Twitter)

Dawei Zhou (University of Illinois at UrbanaChampaign)

Ke Zhou (University of Nottingham)

Feida Zhu (Singapore Management University)

Hengshu Zhu (Baidu Talent Intelligence Center)

Lei Zhu (Huazhong University of Science and Technology)

Xiaofei Zhu (Chongqing University of Technology)

Yadong Zhu (Mobvista)

Yuanyuan Zhu (Wuhan University)

Honglei Zhuang (Google)

Shengyao Zhuang (The University of Queensland)

Imed Zitouni (Google)

Justin Zobel (The University of Melbourne)

Guido Zuccon (The University of Queensland) 


\section{Additional Reviewers}

\begin{tabular}{|c|}
\hline Eashan Adhikarla \\
\hline Youngjun Ahn \\
\hline Firoj Alam \\
\hline Ahmed Al-Baghdadi \\
\hline Milad Alshomary \\
\hline Olga Andreeva \\
\hline Mozhdeh Ariannezhad \\
\hline Fatma Arslan \\
\hline Long Bai \\
\hline Sepehr Bakhshi \\
\hline Yikun Ban \\
\hline Bruno Barbosa \\
\hline Kovan Bavi \\
\hline Samarth Bhargav \\
\hline Jiang Bian \\
\hline Renqin Cai \\
\hline Sergio Canuto \\
\hline Yukun Cao \\
\hline Marco Casavantes \\
\hline Diego Ceccarelli \\
\hline Yusuf Mucahit Cetinkaya \\
\hline Lei Chai \\
\hline Anirban Chakraborty \\
\hline Saurabh Chakravarty \\
\hline Chien-Hung Chen \\
\hline Chong Chen \\
\hline Fumian Chen \\
\hline Hanxiong Chen \\
\hline Huan-Yuan Chen \\
\hline Jiajia Chen \\
\hline Jingjing Chen \\
\hline Long Chen \\
\hline Rocky Chen \\
\hline Wanyu Chen \\
\hline Wei-Fan Chen \\
\hline Zhiyu Chen \\
\hline Ziqi Chen \\
\hline Zixiang Chen \\
\hline Zitong Cheng \\
\hline Yu Chi \\
\hline Zhendong Chu \\
\hline
\end{tabular}

\begin{tabular}{|c|c|}
\hline Luca Ciampi & Shubham Gupta \\
\hline Nachshon Cohen & Soumyajit Gupta \\
\hline Xin Cong & Vinayak Gupta \\
\hline Jean-Valère Cossu & Yu Hao \\
\hline Rajjat Dadwal & Fatima Haouari \\
\hline Enyan Dai & Maram Hasanain \\
\hline Yimeng Dai & Jiafan $\mathrm{He}$ \\
\hline Nicola De Cao & Tianfu He \\
\hline Graziella De Martino & Mingshan Hee \\
\hline Tiago de Melo & Mariya Hendriksen \\
\hline Daniel Xavier de Sousa & Damien Hilloulin \\
\hline Francesco Del Buono & Daeyoung Hong \\
\hline Yang Deng & Seungyeon Hong \\
\hline Vishal Dey & Lu Hongyu \\
\hline Bayu Distiawan & Zhiqiang $\mathrm{Hu}$ \\
\hline Hande Dong & Huinan Huang \\
\hline Sibo Dong & Xinting Huang \\
\hline Yushun Dong & Dmitry Ignatov \\
\hline Zhichao Duan & Israa Jaradat \\
\hline Dimitrios Effrosynidis & Horacio Jarquín \\
\hline Yotam Eshel & Theis Erik Jendal \\
\hline Saher Esmeir & Yiling Jia \\
\hline Ziqing Fan & Tania Jimenez \\
\hline Chuanwen Feng & Huang Jin \\
\hline Patrick Flynn & Shengmin Jin \\
\hline Thibault Formal & Wei Jin \\
\hline Celso França & Georgios Kalamatianos \\
\hline Zuohui Fu & Ehsan Kamalloo \\
\hline Jinhua Gao & Jian Kang \\
\hline Siddhant Garg & Ola Karajeh \\
\hline Garima Gaur & Iman Keivanloo \\
\hline Yingqiang Ge & Eliyahu Kiperwasser \\
\hline Omer Geiger & Deyu Kong \\
\hline Carlos Gemmell & Rohit Kumar \\
\hline Thomas Gérald & Guy Kushilevitz \\
\hline Pouya Ghahramanian & Mucahid Kutlu \\
\hline Harshal Godhia & Suyong Kwon \\
\hline Hanjun Goo & Thi Kim Phung Lai \\
\hline Simon Gottschalk & Alfonso Landin \\
\hline Navita Goyal & Arnon Lazerson \\
\hline Saiping Guan & Yeon-Chang Lee \\
\hline
\end{tabular}


Dan Li

Jiayu Li

Jingzheng Li

Lei Li

Liuqing Li

Qintong Li

Quan Li

Rong-Hua Li

Shijun Li

Xiangju Li

Xiangsheng Li

Xiaoqi Li

Xiuxing Li

Yang Li

Zhenyu Li

Ziming Li

Zixuan Li

Lu Lin

Xixun Lin

Yu-Hsiang Lin

Haochen Liu

Lei Liu

Lihui Liu

Ning Liu

Shuchang Liu

Siwei Liu

Yongqiang Liu

Zhaoyang Liu

Sha Lu

Xuan Lu

Chen Luo

Dan Luo

Yadan Luo

Zhimeng Luo

Jiaqi Ma

Jing Ma

Yufeng Ma

Sean MacAvaney

Stefano Marchesin

Fabio Valerio Massoli

Maanav Mehrotra

Avihai Mejer

Qing Meng
Zaiqiao Meng

Nicola Messina

Paolo Mignone

Vitaly Mirkis

Uma Murthy

Elvis Nava

Yiu-Kai Ng

Sufeng Niu

Alexandros-Fotios Ntogramatzis

Seongwoong $\mathrm{Oh}$

Matteo Paganelli

Yingwei Pan

Zhiqiang Pan

Pradeesh Parameswaran

Ji-Young Park

Antonio Pellicani

Bo Peng

Zhehao Peng

Alberto Purpura

Yifan Qiao

Chuan Qin

Hefei Qiu

Chen Qu

Md Abed Rahman

Niranjan Rai

Clément Rebuffel

Weilong Ren

Xuhui Ren

Myrthe Reuver

Julien Rossi

Kalyani Roy

Sijie Ruan

Stefan Schestakov

Cansu Sen

Janghyuk Seo

Karin Sevegnani

Chen Shani

Jinjin Shao

Dafna Sheinwald

Baoxu Shi

Shaoyun Shi

Tian Shi

Linjun Shou
Anna Shtengel

Georgios Sidiropoulos

Uriel Singer

Ting Su

Xiangguo Sun

Reem Suwaileh

Munira Syed

Symeon Symeonidis

Juntao Tan

Hongyin Tang

Hui Tang

Doga Tekin

Nicolas Tempelmeier

Khushboo Thaker

Hao Tian

Riku Togashi

David Tomás

Liang Tong

Lucia Vadicamo

Asaf Valadarsky

Henry Vieira

Ivo Vigan

David Wang

Jun Wang

Lingxiao Wang

Lu Wang

Shuo Wang

Song Wang

Wei Wang

Wenjie Wang

Wentao Wang

Xi Wang

Yaojing Wang

Yiqi Wang

Zhuo Wang

Zizhe Wang

Jiali Weng

Fangwei Wu

Jiancan $\mathrm{Wu}$

Jun $\mathrm{Wu}$

Xilun Wu

Yue $\mathrm{Wu}$

Yuxia Wu 
Zhijing Wu

Teng Xiao

$\mathrm{Han} \mathrm{Xu}$

Jiacheng Xu

Shengbin Xu

Zhe Xu

Niannan Xue

Erman Yafay

Shiyan Yan

Li Yang

Yaming Yang

Yingrui Yang

Fan Yao

Liuyi Yao

Teng Ye

An-Zi Yen

Sevgi Yigit-Sert
Sevgi Yigit-Sert

Bowen Yu

Junliang Yu

Qu Yue

Fenzhu Zeng

Chen Zhang

Chenchen Zhang

Cheng Zhang

Hao Zhang

Si Zhang

Weijia Zhang

Weitong Zhang

Xiao Zhang

Xuan Zhang

Yang Zhang

Yin Zhang

Yue Zhang
Yuheng Zhang

Kailin Zhao

Tianxiang Zhao

Xiangyu Zhao

Yunxiang Zhao

Geyan Zheng

Jianming Zheng

Lecheng Zheng

Zhiqiang Zhong

Dongruo Zhou

Yao Zhou

Shichao Zhu

Siyuan Zhu

Zhengyuan Zhu

Zhang Zhuoxu

Difan Zou 


\section{Resource Program Committee}

\author{
Alessandro Adamou (The Open University) \\ Qingyao Ai (University of Utah) \\ Vito Walter Anelli (Politecnico di Bari) \\ Alvaro Barreiro (University of A Coruña) \\ Alejandro Bellogin (Universidad Autónoma de Madrid) \\ Rodger Benham (RMIT University) \\ Timo Breuer (TH Köln (University of Applied Sciences)) \\ Ben Carterette (Spotify) \\ David Chaves-Fraga (Universidad Politécnica \\ de Madrid) \\ Chung-Chi Chen (National Taiwan University) \\ Hsin-Hsi Chen (National Taiwan University) \\ Dimitar Dimitrov (GESIS) \\ Zhicheng Dou (Renmin University of China) \\ Lorik Dumani (Trier University) \\ Michael Ekstrand (Boise State University) \\ Nicola Ferro (University of Padova) \\ Tirthankar Ghosal (Charles University) \\ Lorraine Goeuriot (Université Grenoble Alpes) \\ Cathal Gurrin (Dublin City University) \\ Allan Hanbury (Vienna University of Technology) \\ Claudia Hauff (Delft University of Technology) \\ Xiangnan He (University of Science and Technology \\ of China) \\ Minlie Huang (Tsinghua University) \\ Gareth Jones (Dublin City University) \\ Chris Kamphuis (Radboud University) \\ Makoto P. Kato (University of Tsukuba) \\ Johannes Kiesel (Bauhaus-Universität Weimar)
}

Benjamin Lee (University of Washington)

Xiaolu Lu (Microsoft)

Jiyun Luo (Pinterest)

Weizhi Ma (Tsinghua University)

Stuart Mackie (University of Strathclyde)

Antonio Mallia (New York University)

Jiaxin Mao (Renmin University of China)

Noemi Mauro (University of Torino)

Alistair Moffat (The University of Melbourne)

Hiroaki Ohshima (University of Hyogo)

David Otero (University of A Coruña)

Iadh Ounis (University of Glasgow)

Marinella Petrocchi (IIT-CNR)

Karen Pinel-Sauvagnat (IRIT)

Julian Risch (Hasso-Plattner-Institut)

Ahmad Sakor (L3S Research Center)

Mete Sertkan (Vienna University of Technology)

Damiano Spina (RMIT University)

Zhiwen Tang (Georgetown University)

Johanne R Trippas (The University of Melbourne)

Zijie J. Wang (Georgia Tech)

Tim Weninger (University of Notre Dame)

Takehiro Yamamoto (University of Hyogo)

Linyi Yang (UCD)

Andrew Yates (Max Planck Institute for Informatics)

Zhaohao Zeng (Waseda University)

Fan Zhang (Tsinghua University)

Xinyi Zhou (Syracuse University) 


\section{Perspectives Program Committee}

Nicholas Belkin (Rutgers University)

Ben Carterette (Spotify)

Charles Clarke (University of Waterloo)

Nick Craswell (Microsoft)

Susan Dumais (Microsoft)

Michael Ekstrand (Boise State University)

Norbert Fuhr (University of Duisburg-Essen)

Donna Harman (NIST)

Diane Kelly (University of Tennessee)

Rishabh Mehrotra (Spotify Research)

Alistair Moffat (The University of Melbourne)

Mark Smucker (University of Waterloo)

Ian Soboroff (NIST)

Jaime Teevan (Microsoft)

Ellen Voorhees (NIST)

Min Zhang (Tsinghua University)

\section{Workshop Program Committee}

Alberto Barrón-Cedeño (University of Bologna)

Muthu Chandrasekaran (Amazon)

Hongshen Chen (Data Science Lab, FD.com)

Paul Groth (University of Amsterdam)

Dietmar Jannach (University of Klagenfurt)

Haiming Liu (University of Bedfordshire)

Jaime Lorenzo-Trueba (Universidad Politecnica de Madrid)

Cheng Luo (Tsinghua University)

Philipp Mayr (GESIS)

Massimo Melucci (University of Padova)

Xi Sunshine Niu (UNC Charlotte)

Tony Russell-Rose (Goldsmiths - University of London)

Elena Sokolova (Amazon research)

Andrew Trotman (University of Otago)

Dawei Yin (FD.com)

Hamed Zamani (Microsoft)

Min Zhang (Tsinghua University)

Yating Zhang (Alibaba) 


\section{Tutorial Program Committee}

Qingyao Ai (University of Utah)

Avishek Anand (L3S Research Center)

Ricardo Campos (Ci2 - Polytechnic Institute of Tomar, INESC TEC)

Engin Demir (Hacettepe University)

Stefan Dietze (GESIS - Leibniz Institute for the Social Sciences)

Zhicheng Dou (Renmin University of China)

Nattiya Kanhabua (Upwork)

Pinar Karagoz (Middle East Technical University)

Joel Mackenzie (The University of Melbourne)

Graham Mcdonald (University of Glasgow)

Franco Maria Nardini (ISTI-CNR)

Nicola Tonellotto (University of Pisa)

Chenyan Xiong (Microsoft)

\section{Doctoral Consortium Program Committee}

Eugene Agichtein (Emory University)

Ioannis Arapakis (Telefonica Research)

James Caverlee (Texas A\&M University)

Kevyn Collins-Thompson (University of Michigan)

Susan Dumais (Microsoft)

Nicola Ferro (University of Padova)

Luanne Freund (The University of British Columbia)

Lorraine Goeuriot (Université Grenoble Alpes)

Hideo Joho (University of Tsukuba)

Jaap Kamps (University of Amsterdam)

Diane Kelly (University of Tennessee)

Oren Kurland (Technion, Israel Institute of Technology)

Weizhi Ma (Tsinghua University)

Ana Gabriela Maguitman (Universidad Nacional del Sur)

Jeanna Matthews (Clarkson University)

Heather O'Brien (The University of British Columbia)

Filip Radlinski (Google)

Fiana Raiber (Yahoo Research)

Pengjie Ren (Shandong University)

Yongfeng Zhang (Rutgers University) 


\section{Demonstration Program Committee}

Khaled Ammar (BorealisAI)

Alejandro Bellogin (Universidad Autónoma de Madrid)

B. Barla Cambazoglu (RMIT University)

Ricardo Campos (Ci2 - Polytechnic Institute of Tomar, INESC TEC)

Meng Chen (Shandong University)

Xiaocong Chen (University of New South Wales)

Arjen de Vries (Radboud University)

Li He (FD.COM)

Chaoran Huang (University of New South Wales)

Jussi Karlgren (Spotify)

Udo Kruschwitz (University of Regensburg)

Jochen L. Leidner (Refinitiv Labs and University of Sheffield)

Lei Liu (York University)

Yong Liu (Nanyang Technological University)

David Losada (University of Santiago de Compostela)

Edgar Meij (Bloomberg L.P.)

Franco Maria Nardini (ISTI-CNR)

Minh C. Phan (Microsoft)

Jeremy Pickens (OpenText)

Zhaochun Ren (Shandong University)

Shuming Shi (Tencent)

Fabrizio Silvestri (Facebook)

Damiano Spina (RMIT University)

Jianshu Weng (Swiss Re)

Takehiro Yamamoto (University of Hyogo)

Lina Yao (The University of New South Wales)

Xingdi Yuan (Microsoft)

Shuai Zhang (ETH Zurich)

Bolong Zheng (Aalborg University)

Lixin Zou (Tsinghua University) 


\section{SIGIR 2021 Sponsor \& Supporters}

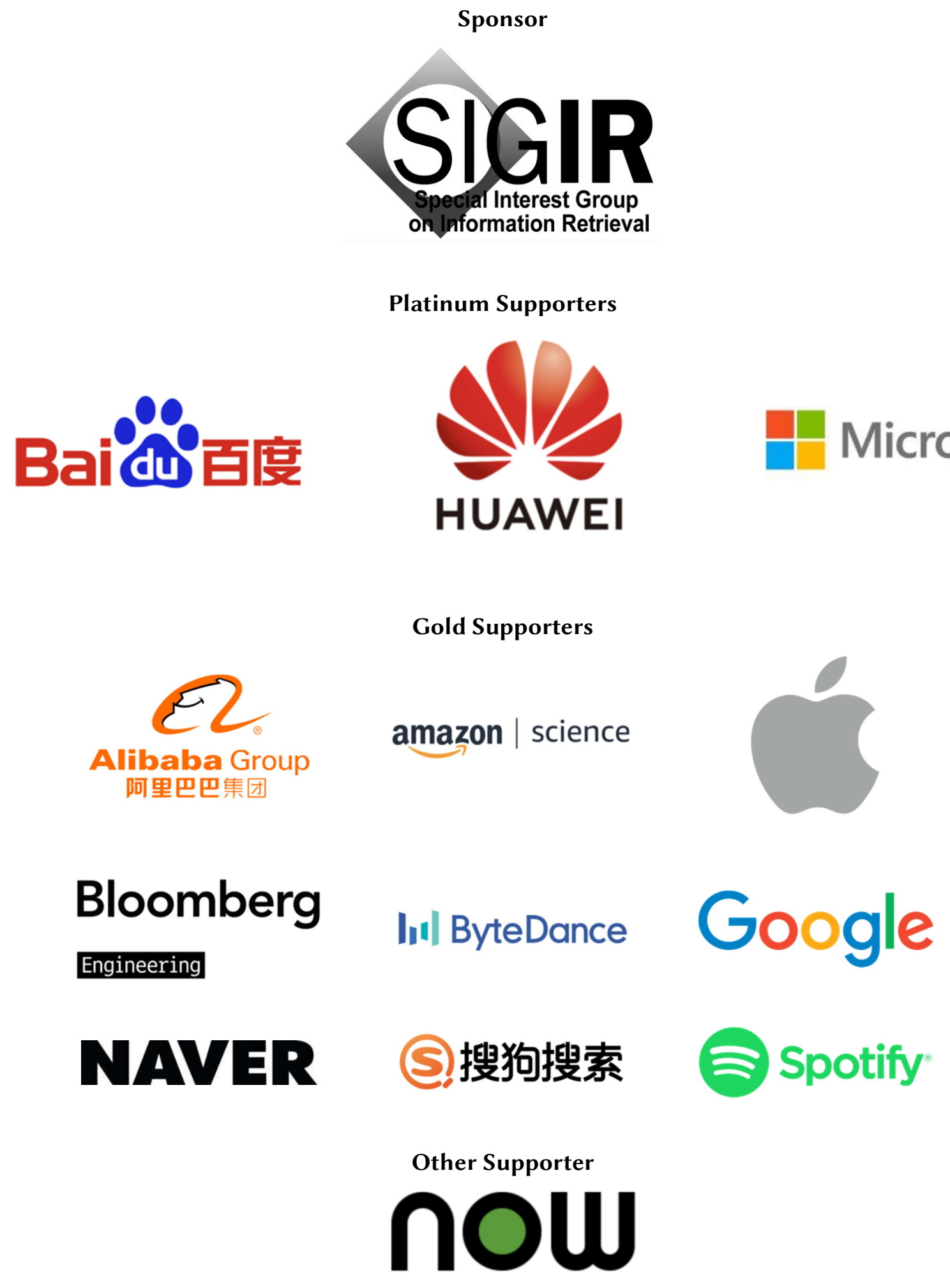


This author profile is generated by Scopus Learn more

Jimmy,

(1) Universitas Surabaya, Surabaya, Indonesia

https:///orcid.org/0000-0002-5376-769X

Edit profile $\triangle$ Set alert 을 Potential author matches —- Export to SciVal

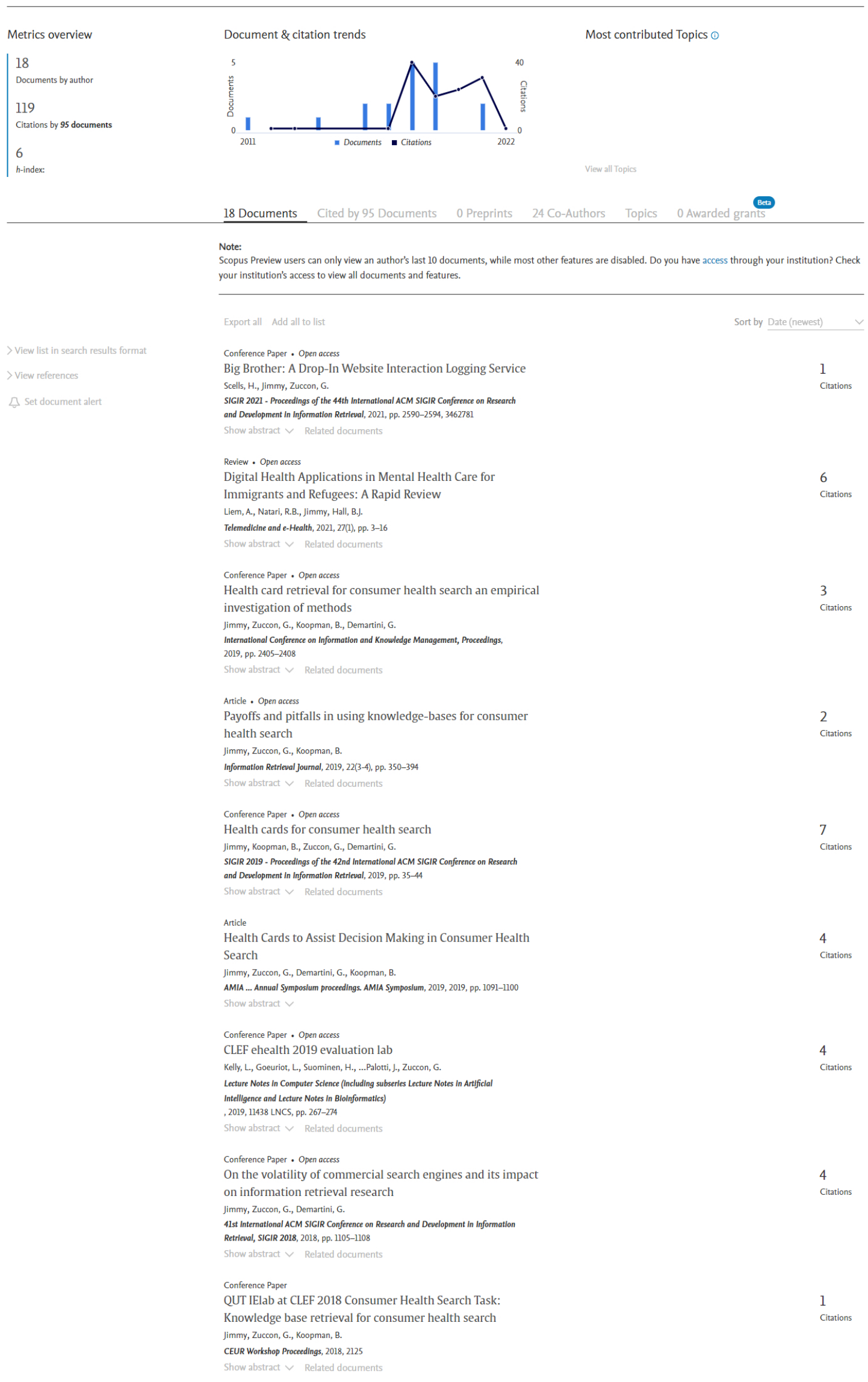


Conference Paper

Overview of the CLEF 2018 consumer health search task

mmy, Zuccon, G., Palotti, J., Goeuriot, L., Kelly, L

CEUR Workshop Proceedings, 2018, 2125

CEUR Workshop Proceedings, 2018, 2125

Back to top

$\begin{array}{lll}\text { About Scopus } & \text { Language } & \text { Customer Service } \\ \text { What is Scopus } & \text { 日本語に切り替える } & \text { Help } \\ \text { Content coverage } & \text { 切换到简体中文 } & \text { Tutorials } \\ \text { Scopus blog } & \text { 切換到等體中文 } & \text { Contact us } \\ \text { Scopus API } & \text { Русский язык } & \\ \text { Privacy matters } & & \end{array}$

ELSEVIER

Terms and conditions $\pi$ Privacy policy $\pi$

Copyright (@) Elsevier B.V ^. All rights reserved. Scopus $₫$ is a registered trademark of Elsevier B.V.

We use cookies to help provide and enhance our service and tailor content. By continuing, you agree to the use of cookies.

QRELX 
This aythor profile is generaied ty Soppus terarn mary

Jimmy,

(1) Univinsitas Surataya, Surabaya, Indonesia

(1) Hetps:/forcid.org/0000-0002-5376-769X

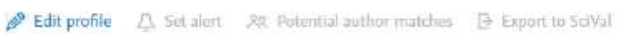

Metrics overview
\begin{tabular}{|l}
18 \\
Documents by author \\
119 \\
Ctations by 95 documents \\
6 \\
$h$-index
\end{tabular}

\section{Document \& citation trends}

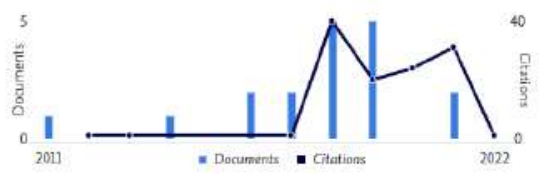

Most contributed Topics $\varnothing$

\section{Viow all Topita}

\section{Documents Gited by 95 Documents OPreprints 24 Co-Authors Topics DAwarded grant5}

Note:

Scopus Preview users can only view an author's last 10 documents, while most other features are disabled. Do you have access through your institution? Check your institution's access to view all documents and feazures:

Export all Add att to lin:

Sort by Dute (nnewest)

Conference Paper - Open access

Big Brother: A Drop-In Website Interaction Logging Service $\quad 1$

Scells, H., Jimmy, Zuccon, C.

Citations

SIGIR 2021 . Procendings of the 44th heterenational ACM SIGIR Conference

and Dowlotoment in infornution Retricual, 2027, pp., 2590-2594, 3662781

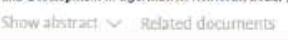

Review - Opero access

Digital Health Applications in Mental Health Care for

Immigrants and Refugees: A Rapid Review

Llem, A, Natar, R.B., Jimmy, Hall, B...

Gintions

Telementiline and $s-H$ thath, 202L, 27(1), pp. 3-16

Show absirsat $\checkmark$ Related documents 